Universidade de São Paulo

Instituto de Astronomia, Geofísica e Ciências Atmosféricas

Departamento de Astronomia

Diana Renata Gonçalves Gama

\title{
Ambiente Molecular e Formação Estelar Associados à Bolha Infravermelha N10
}

São Paulo

2016 



\section{Diana Renata Gonçalves Gama}

\section{Ambiente Molecular e Formação Estelar Associados à Bolha Infravermelha N10}

Tese apresentada ao Departamento de Astronomia do Instituto de Astronomia, Geofísica e Ciências Atmosféricas da Universidade de São Paulo como requisito parcial para a obtenção do título de Doutora em Ciências.

Área de Concentração: Astronomia

Orientador: Prof. Dr. Jacques Lépine

Versão Corrigida. A original encontra-se disponível na Unidade.

São Paulo

2016 

Aos meus pais Mercedes e Dimas. 



\section{Agradecimentos}

Agradeço à minha família e aos meus amigos. Aos colegas, professores e funcionários do Departamento de Astronomia do IAG/USP.

Ao meu orientador Prof. Jacques Lépine, ao meu relator Prof. Amaury Almeida e aos professores que fizeram parte da minha formação durante minha pós graduação.

Aos pesquisadores que, de alguma forma, contribuíram para esse trabalho: Profa. Yuefang Wu, Dr. Tie Liu, Dr. Fanyi Meng, Dr. Jinghua Yuan, Profa. Zulema Abraham, Prof. Roberto Costa, Prof. Bertrand Lefloch, Profa. Cristina Cappa, Dr. Lokesh Dewangan, Dr. Edgar Mendoza, Dr. Nicolás Duronea.

À National Natural Science Foundation of China, por apoiar financeiramente nosso trabalho durante minha estadia em Pequim, sob o projeto NSFC 11373009-11433008. Aos funcionários do telescópio IRAM 30-m pela hospitalidade e assistência durante as observações em Granada, Espanha.

À CAPES, pelo apoio financeiro sob forma de cota institucional. Ao CNPq, pelo apoio financeiro sob o projeto $\mathrm{n}^{\circ} 870199 / 1997-8$.

Esta tese foi escrita em LTEX com a classe IAGTESE, para teses e dissertações do IAG. 

"Fecho meus olhos, e os volto para o meu coração, Como um homem que pede vinho antes de lutar, Visão mais feliz, de outro tempo, eu quis saborear Para ficar mais apto a encarar minha missão. Pensar antes, lutar depois, eis do soldado o bordão: Um vislumbre do passado pode a tudo acertar." Robert Browning 



\section{Resumo}

Realizamos um estudo multi-comprimento de onda do ambiente ao redor da bolha infravermelha N10, usando os telescópios PMO 13.7-m, IRAM 30-m, APEX e o arranjo VLA. Bolhas infravermelhas são regiões ideais para investigar o impacto da radiação UV sobre o material molecular. Nosso objeto de estudo é a bolha N10, que apresenta uma região HII em seu interior. Estudos no IV sobre o conteúdo estelar jovem em N10 sugerem um cenário de formação estelar contínua, possivelmente desencadeada, na borda da região HII. Realizamos observações da transição $J=1-0$ das espécies ${ }^{12} \mathrm{CO}$ e ${ }^{13} \mathrm{CO}$ na direção de $\mathrm{N} 10$, utilizando o telescópio PMO 13.7-m. Realizamos também observações com o telescópio IRAM 30-m para investigar moléculas traçadoras de formação estelar. Analisamos as emissões em $24 \mu \mathrm{m}, 8.0 \mu \mathrm{m}, 20 \mathrm{~cm}$ e $870 \mu \mathrm{m}$ a partir de dados da literatura. Duas condensações foram identificadas em ${ }^{13} \mathrm{CO}$, para as quais estimamos propriedades como dimensão, temperatura, densidade e massa. Calculamos o fluxo de fótons ionizantes e a densidade eletrônica no interior da bolha, e obtivemos o tempo de fragmentação para N10. Estimamos as propriedades físicas para o clump de poeira fria mais denso da bolha. Identificamos YSOs e calculamos os parâmetros dos objetos Classe I. Emissão das transições $\mathrm{HCO}^{+}(1-0), \mathrm{HCN}(1-0), \mathrm{SiO}(2-1), \mathrm{N}_{2} \mathrm{H}^{+}(1-0)$ e CS (3-2) foram mapeadas. Realizamos também integração profunda na direção do clump mais denso de N10 para investigar sua complexidade química. A idade dinâmica de N10 é menor do que o tempo de fragmentação estimado, portanto a formação estelar desencadeada deve ocorrer pelo mecanismo "RadiationDriven Implosion”. A distribuição espacial das espécies moleculares revelaram características de formação estelar em N10. O levantamento de linhas espectrais na direção do clump mais denso mostrou uma grande complexidade química na região, e confirma a presença de outflows e atividade de formação estelar em N10. 



\section{Abstract}

We performed a multi-wavelength study of the environment surrounding the infrared bubble N10 by using the telescopes PMO 13.7-m, IRAM 30-m, APEX and the VLA array. Infrared bubbles are ideal regions to investigate the impact of UV radiation on the molecular material. Our target is the N10 bubble, which hosts an HII region. IR studies on the young stellar content in N10 suggest a scenario of ongoing star formation, possibly triggered, at the edge of the HII region. We carried out observations of the transition $J=1-0$ of the species ${ }^{12} \mathrm{CO}$ and ${ }^{13} \mathrm{CO}$ towards N10 using the PMO 13.7-m telescope. We also conduct observations with the IRAM 30-m telescope to investigate molecules that trace star formation. We analyzed the emissions at $24 \mu \mathrm{m}, 8.0 \mu \mathrm{m}, 20 \mathrm{~cm}$ and $870 \mu \mathrm{m}$ from the literature. Two condensations were identified at ${ }^{13} \mathrm{CO}$ emission, for which we derived properties such as size, temperature, density, and mass. We calculated the flux of ionizing photons and the electronic density inside the bubble, and we derived the fragmentation time to N10. We estimated the physical properties for the densest cold dust clump in the bubble. We identified the YSOs and derived the parameters for the Class I objects. Emission of the transitions $\mathrm{HCO}^{+}(1-0), \mathrm{HCN}(1-0), \mathrm{SiO}(2-1), \mathrm{N}_{2} \mathrm{H}^{+}(1-0)$ and CS $(3-2)$ were mapped. We also performed a deep integration towards the densest clump in N10 to investigate its chemical complexity. The dynamic age of N10 is lower than the estimated fragmentation time, therefore triggered star formation should occur by "Radiation-Driven Implosion" mechanism. The spatial distribution of the molecular species revealed characteristics of star formation in N10. The survey of spectral lines towards the densest clump shows a high chemical complexity, and confirms the presence of outflows and star formation activity in N10. 



\section{Lista de Figuras}

1.1 Esquema dos estágios da evolução de objetos estelares jovens, de acordo com suas denominações no milimétrico/infravermelho. (Feigelson \& Montmerle, 1999). 29

1.2 Exemplo de SED de um objeto YSO Classe I. A curva em preto representa a emissão total do YSO e a linha tracejada é a curva de corpo negro. A contribuição do envoltório é evidenciada pelo excesso de emissão no infravermelho distante. (Figura adaptada de Wilking, 1989). . . . . . . . . . . . . . . . . 30

1.3 Exemplo de SED de um objeto estelar jovem Classe II. (Figura adaptada de Wilking, 1989). . . . . . . . . . . . . . . . . . . 31

1.4 Exemplo de SED de um Disco de Transição. A distribuição se assemelha a um corpo negro estelar, atingindo seu máximo em comprimentos de onda ópticos ou infravermelhos. Excesso no infravermelho médio ainda é muitas vezes observado, possivelmente devido ao disco fino restante. (Figura adaptada de Wilking, 1989).

1.5 Esquema de uma PDR iluminada por um forte campo de radiação pela esquerda. A PDR se extende desde a região de transição $\mathrm{H}^{+} / \mathrm{H}$, passando pelas transições $\mathrm{H} / \mathrm{H}_{2}$ e $\mathrm{C}^{+} / \mathrm{C} / \mathrm{CO}$ até a fronteira $\mathrm{O} / \mathrm{O}_{2}$. (Imagem adaptada de Hollenbach \& Tie-

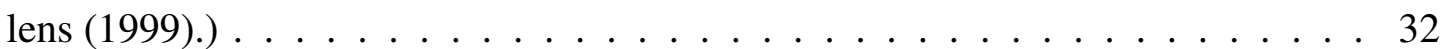

1.6 Esquema de uma região HII simétrica em expansão, inserida em um meio homogêneo. Nesse modelo, a região HII é esférica e sua porção ionizada é rodeada pelo material denso e neutro, que foi coletado durante a fase de expansão. (Figura adaptada de Deharveng et al. 2010). . . . . . . . . . . . . . 34 
1.7 Perspectiva esquemática de uma região HII esférica em expansão e ambiente neutro ao redor. Diversos processos de formação estelar desencadeada podem ser caracterizados: 1 - instabilidades gravitacionais em pequena escala; 2 - instabilidades gravitacionais em larga escala, levando à formação de fragmentos de alta massa; 3 - radiação ionizante agindo no meio turbulento; 4 - compressão de clumps densos pré-existentes, conduzida por radiação (Figura de Deharveng et al. 2010). . . . . . . . . . . . . . . . . . . 36

2.1 Imagem de N10 em $8.0 \mu \mathrm{m}$, localizada a uma distância de $4.7 \mathrm{kpc}$. A cruz preta indica o centro da região HII dentro da bolha infravermelha. A elipse vermelha indica a borda de N10. A escala corresponde a um campo de 5 pc ( 3.7 arcmin). A elipse branca pontilhada indica a posição de N11, cujo centro está localizado em $l=13.218^{\circ}, b=0.082^{\circ}$, aqui identificado com um "x"em preto.

2.2 Composição em três cores da bolha N10 com imagens Spitzer-GLIMPSE 3.6 $\mu \mathrm{m}$ (em azul), $4.5 \mu \mathrm{m}$ (em verde) e $8.0 \mu \mathrm{m}$ (em vermelho). A posição das estrelas identificadas por Watson et al. (2008) estão marcadas em estrelas amarelas; condensações de poeira identificadas por Deharveng et al. (2010) são identificadas por quadrados magenta (\#1 ao lado direito, \#2 ao lado esquerdo); diamantes em ciano representam a posição dos masers de metanol por Szymczak et al. (2000) acima e Pandian et al. (2008) abaixo. . . . . . . . . . . . . .

2.3 Imagens multi-comprimento de onda da região N10. Os painéis mostram a emissão em $4.5 \mu \mathrm{m}, 5.8 \mu \mathrm{m}, 8.0 \mu \mathrm{m}, 24 \mu \mathrm{m}, 870 \mu \mathrm{m}$ e $20 \mathrm{~cm}$ na direção da região de interesse, dos levantamentos Spitzer-GLIMPSE, Spitzer-MIPSGAL, APEX/ATLASGAL e GPS-VLA, respectivamente. Todas as imagens mostram a mesma região do céu, no primeiro painel, a cruz branca marca o centro da região HII; em todas as imagens a elipse vermelha é para referência, conforme mostrado na Figura $2.1 \ldots \ldots \ldots \ldots \ldots$

3.1 Transmissão atmosférica entre 60 e $400 \mathrm{GHz}$ para dois valores de vapor d'água precipitável (pwv, do inglês precipitable water vapor): $2 \mathrm{~mm}$ em azul e $4 \mathrm{~mm}$ em vermelho. As bandas EMIR são mostradas abaixo, com as frequências de algumas transições moleculares importantes. . . . . . . . . . . . . . 49 
4.1 Linhas espectrais. A coluna à esquerda mostra o espectro médio da região observada, para ${ }^{12} \mathrm{CO}(1-0)$ no painel acima e para ${ }^{13} \mathrm{CO}(1-0)$ no painel abaixo. A coluna à direita apresenta o espectro dessas duas linhas na posição do pico de emissão, na posição $\alpha_{2000}=18^{h} 14^{m} 10^{s} .03$ e $\delta_{2000}=-17^{\circ} 27^{\prime} 35^{\prime \prime} .3\left(l=13.21^{\circ}\right.$,

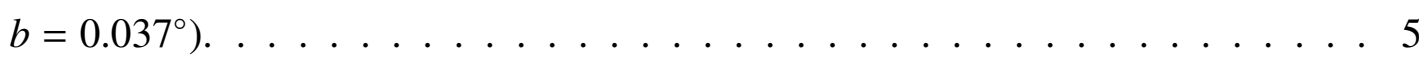

4.2 Mapas de canal da emissão ${ }^{12} \mathrm{CO}(J=1-0)$ em contornos sobrepostos à imagem Spitzer $8.0 \mu \mathrm{m}$. Os contornos começam em $5 \sigma$, com passos de $3 \sigma(1 \sigma=$ $0.90 \mathrm{~K} \mathrm{~km} \mathrm{~s}^{-1}$ ). A velocidade principal em $\mathrm{km} \mathrm{s}^{-1}$ é indicada no canto superior direito em cada painel menor. A barra de intensidade ao lado direito mostra a intensidade do fluxo da emissão em $8.0 \mu \mathrm{m}$ em escala logarítmica e o círculo pequeno no último mapa representa o tamanho do feixe (beam size). . . . . . . . 53

4.3 O mesmo que a Figura 4.2, para a emissão ${ }^{13} \mathrm{CO}(J=1-0)$. Os contornos começam em $5 \sigma$, aumentando com um passo de $3 \sigma\left(1 \sigma=0.55 \mathrm{~K} \mathrm{~km} \mathrm{~s}^{-1}\right)$. . . 54

4.4 Os painéis acima mostram os contornos da emissão ${ }^{12} \mathrm{CO}$ centrada em $20 \mathrm{~km}$ $\mathrm{s}^{-1}$ (à esquerda), $37 \mathrm{~km} \mathrm{~s}^{-1}$ (no centro) e $52 \mathrm{~km} \mathrm{~s}^{-1}$ (à direita), de 4.5 a $12.6 \mathrm{~K}$ com passos de $2.7 \mathrm{~K}$. Os painéis abaixo apresentam contornos da distribuição da emissão ${ }^{13} \mathrm{CO}$ em $20 \mathrm{~km} \mathrm{~s}^{-1}$ (à esquerda), $37 \mathrm{~km} \mathrm{~s}^{-1}$ (no centro) e $52 \mathrm{~km} \mathrm{~s}^{-1}$ (à direita), de 2.75 a $11 \mathrm{~K}$ com passos de $1.65 \mathrm{~K}$. Todos os painéis exibem a imagem Spitzer $8.0 \mu \mathrm{m}$ como plano de fundo. . . . . . . . . . . . 5 56

4.5 Região dos clumps identificados no mapa de intensidade de ${ }^{13} \mathrm{CO}$ centrado em 52 $\mathrm{km} \mathrm{s}^{-1}$, em escala de cinza. O Clump \#1 e o Clump \#2 parecem estar fisicamente associados à bolha N10. Contornos em preto mostram também a emissão em ${ }^{13} \mathrm{CO}$ de 2.75 a $11 \mathrm{~K}$, em passos de $1.65 \mathrm{~K}$. A cruz preta indica o centro da região

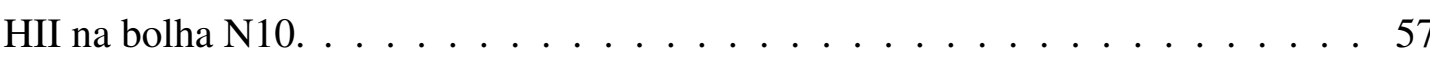

4.6 Panel superior: emissão $870 \mu \mathrm{m}$ mostrando a distribuição de poeira fria em

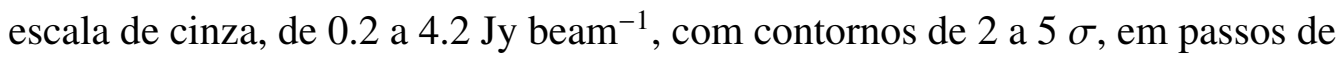
$1 \sigma\left(1 \sigma=0.5 \mathrm{Jy}^{-1}\right.$ beam $\left.^{-1}\right)$. Painel inferior: imagem Spitzer $8.0 \mu \mathrm{m}$ ao fundo, com os mesmos contornos em $870 \mu \mathrm{m}$ da figura à esquerda. Nas duas imagens, o ponto em preto representa o beam size. . . . . . . . . . . . . 62

4.7 Mapa em escala de cinza da emissão $870 \mu \mathrm{m}$, com os mesmos contornos da Figura 4.6. O retângulo em vermelho destaca a região do clump de poeira fria mais denso, para o qual os parâmetros físicos foram obtidos. 
4.8 Painel superior: intensidade da emissão em $20 \mathrm{~cm}$ em escala de cinza, de 0 a 22 mJy beam ${ }^{-1}$, com contornos começando em $2 \sigma$ em passos de $2 \sigma(1 \sigma=2.8$ mJy beam $\left.{ }^{-1}\right)$. Painel inferior: imagem RGB Spitzer em plano de fundo $(3.6 \mu \mathrm{m}$ em azul, $4.5 \mu \mathrm{m}$ em verde e $8.0 \mu \mathrm{m}$ em vermelho) com mesmos contornos que o painel superior. Estrelas amarelas marcam a posição das candidatas a estrelas ionizantes listadas na Tabela $2.1 \ldots \ldots \ldots$. . . . . . . . . . . . 65

4.9 Distribuição da emissão em $24 \mu \mathrm{m}$ em escala de cinza de 0 a $1500 \mathrm{MJy} \mathrm{sr}^{-1} \mathrm{com}$ os mesmos contornos em $20 \mathrm{~cm}$ da Figura $4.8 \ldots \ldots 6$

4.10 Diagrama cor-cor w1-w2 versus w2-w3, de candidatos a YSOs ao redor de N10. Cruzes vermelhas: objetos Classe I; círculos verdes: fontes Classe II; quadrados azuis: Discos de Transição. As linhas tracejadas indicam os limites das regiões de acordo com KL. Os Discos de Transição não são classificados definitivamente neste diagrama. Os objetos restantes são indicados por pontos pretos. . . . . . . . 69

4.11 Diagrama cor-cor w1-w2 versus w3-w4, parecido com a Figura 4.10. O locus usado para definir os Discos de Transição (quadrados azuis) também contém muitos objetos Classe II (círculos verdes), pois esses objetos foram classificados como Classe II no passo anterior que utiliza outro diagrama cor-cor (Fig. 4.10). . 70

4.12 Distribuição dos YSOs Classe I (cruzes vermelhas), Classe II (círculos verdes) e Discos de Transição (diamantes azuis). Os contornos em preto mostram a distribuição da componente em $52 \mathrm{~km} \mathrm{~s}^{-1}$ do ${ }^{13} \mathrm{CO}$ e os contornos em amarelo representam a emissão em $870 \mu \mathrm{m} . \ldots \ldots \ldots \ldots \ldots$. . . . . . . . . . . .

4.13 SEDs ajustadas para YSOs Classe I na região de N10. Os círculos preenchidos mostram os fluxos de entrada. A linha preta mostra o melhor ajuste. A linha pontilhada mostra a fotosfera estelar correspondente à fonte central de acordo com o modelo de melhor ajuste, como seria na ausência de poeira circunstelar (mas incluindo a extinção interestelar) . . . . . . . . . . . . . 72 
4.14 Análise preliminar da detecção de $\mathrm{HCO}^{+}(1-0)$ em coordenadas equatoriais. $\mathrm{O}$ painel inferior esquerdo mostra o mapa de intensidade integrada de 30 a $70 \mathrm{~km}$ $\mathrm{s}^{-1}$; o espectro médio da região correspondente à intensidade integrada de $30 \mathrm{a}$ $70 \mathrm{~km} \mathrm{~s}^{-1}$ encontra-se no painel inferior direito. O painel superior direito mostra o espectro no pico de emissão identificado no mapa de intensidade integrada; o painel superior esquerdo mostra o mapa de intensidade que corresponde apenas àquele canal, de acordo com a velocidade do pico de emissão. . . . . . . . . . . . 75

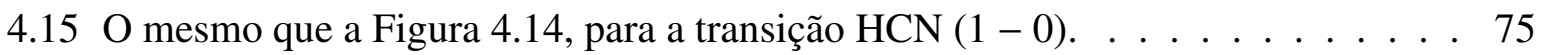

$4.16 \mathrm{O}$ mesmo que a Figura 4.14 para a transição $\mathrm{SiO}(2-1) \ldots \ldots$. . . . . . . 76

$4.17 \mathrm{O}$ mesmo que a Figura 4.14, para a transição $\mathrm{N}_{2} \mathrm{H}+(1-0) \ldots \ldots \ldots$

4.18 O mesmo que a Figura 4.14 para a transição CS $(3-2)$. . . . . . . . . . . 77

4.19 Distribuição espacial de $\mathrm{HCO}^{+}(1-0)$ na direção da bolha N10 com contornos de $30 \%$ a $90 \%$ do pico de intensidade $\left(T_{\text {peak }}=1.14 \mathrm{~K}\right)$ em passos de $10 \%$ de $T_{\text {peak }}$. 78

4.20 Distribuição espacial de HCN ( 1 - 0) na direção da bolha N10 com contornos de $30 \%$ a $90 \%$ do pico de intensidade $\left(T_{\text {peak }}=0.53 \mathrm{~K}\right)$ em passos de $10 \%$ de $T_{\text {peak }}$. 79

4.21 Distribuição espacial de $\mathrm{SiO}(2-1)$ na direção da bolha N10 com contornos de $10 \%$ a $90 \%$ do pico de intensidade $\left(T_{\text {peak }}=0.25 \mathrm{~K}\right)$ em passos de $10 \%$ de $T_{\text {peak }}$. 79

4.22 Distribuição espacial de $\mathrm{N}_{2} \mathrm{H}+(1-0)$ na direção da bolha N10 com contornos de $20 \%$ a $90 \%$ do pico de intensidade $\left(T_{\text {peak }}=1.14 \mathrm{~K}\right)$ em passos de $10 \%$ de $T_{\text {peak }}$. 80

4.23 Distribuição espacial de CS (3 - 2) na direção da bolha N10 com contornos de $20 \%$ a $90 \%$ do pico de intensidade $\left(T_{\text {peak }}=0.96 \mathrm{~K}\right)$ em passos de $10 \%$ de $T_{\text {peak }}$. . 80

4.24 Mapa da distribuição de $8.0 \mu \mathrm{m}$, em escala de cinza. Os contornos em magenta, indicando a distribuição de $\mathrm{HCO}^{+}(1-0)$, são os mesmos da Figura 4.19. Os contornos em amarelo mostram a emissão de $\mathrm{SiO}(2-1)$, em passos iguais aos da Figura 4.23, As estrelas são as candidatas a fontes ionizantes citadas na Tabela 2.1, 81

4.25 Painel superior: perfil de linha de $\mathrm{HCO}^{+}(1-0)$. Painel inferior: perfil de linha de $\mathrm{SiO}(2-1)$. Ambos na direção do pico de emissão, com intensidade em Ta (K). 82

4.26 Espectro de integração profunda na direção da condensação molecular mais

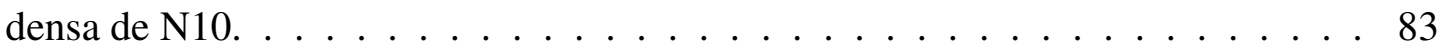


5.1 Melhor ajuste para um modelo de três braços espirais da Galáxia para a distribuição de regiões HII, representadas pelos quadrados vermelhos. A estrela em preto acima indica a posição do Sol e a outra estrela preta indica o centro da Galáxia. A elipse vermelha aberta indica a barra Galáctica e a elipse preenchida em cinza mostra o melhor ajuste para o Braço 3 kpc Próximo e Braço 3 kpc Distante. As linhas tracejadas indicam as direções tangenciais observadas. O diamante em azul mostra a posição aproximada da bolha N10 no plano da Galáxia. Figura

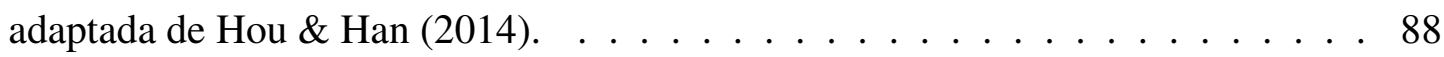




\section{Lista de Tabelas}

1.1 Fases do MI e suas propriedades físicas. Adaptado de Kwok (2007). . . . . . . . 26

1.2 Propriedades de nuvens moleculares, clumps e cores estelares. (Tabela adaptada de Bergin \& Tafalla, 2007) . . . . . . . . . . . . . . . . 27

2.1 Estrelas candidatas a fontes ionizantes no sistema da bolha N10. . . . . . . . . 42

3.1 Tamanho do feixe à meia potência (HPBW) para cada linha observada no te-

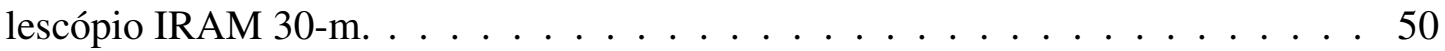

4.1 Velocidades obtidas na direção da bolha N10, através de diferentes métodos. . . . 55

4.2 Parâmetros das linhas dos espectros médios da região observada, ilustrados na coluna esquerda na Figura $4.1 \ldots \ldots \ldots \ldots$

4.3 Parâmetros calculados para os clumps observados em ${ }^{13} \mathrm{CO}$. . . . . . . . . . . 60

4.4 Parâmetros físicos obtidos a partir do modelo de Robitaille et al. (2007), para objetos Classe I candidatos a associados. . . . . . . . . . . . . . . . . . 73

4.5 Espécies moleculares detectadas na direção da bolha N10 . . . . . . . . . . 74

4.6 Conteúdo molecular detectado no levantamento de linhas espectrais. . . . . . . . 84

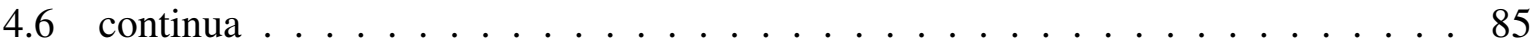

4.7 Classificação das moléculas detectadas de acordo com suas propriedades. . . . . 86

A.1 Candidatos a objetos estelares jovens (YSOs) na direção da região N10. . . . . . 109

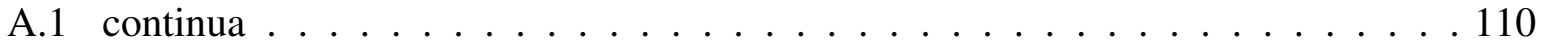

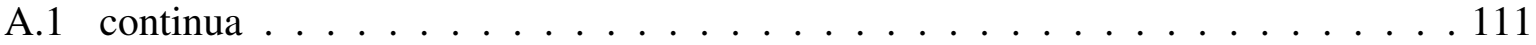

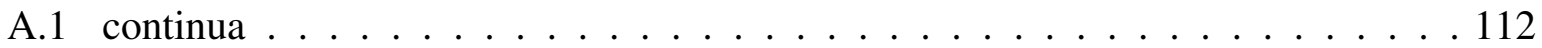

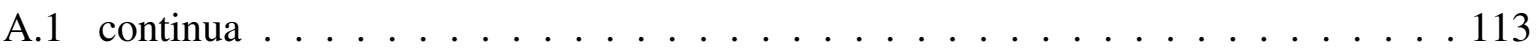




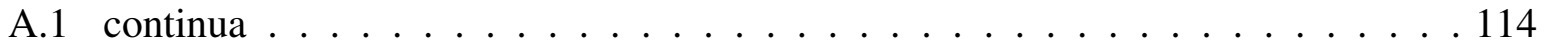




\section{Lista de acrônimos}

- APEX: Atacama Pathfinder EXperiment

- ATLASGAL: APEX Telescope Large Area Survey of the Galaxy

- CLASS: Continuum and Line Analysis Single-dish Software

- COM: Complex Organic Molecule

- EMIR: Eight MIxer Receiver

- FFTS: Fast Fourier Transform Spectrometer

- FWHM: Full Width at Half Maximum

- FUV: Far-Ultraviolet

- HPBW: Half Power Beam Width

- GILDAS: Grenoble Image and Line Data Analysis Software

- GLIMPSE: Galactic Legacy Infrared Midplane Extraordinaire

- GreG: Grenoble Graphic

- IR: Infrared

- IRAM: Institut de Radioastronomie Millimétrique

- IRDC: Infrared Dark Clouds

- IV: Infravermelho 
- KDA: Kinematic Distance Ambiguity

- LSR: Local Standard of Rest

- LTE: Local Thermal Equilibrium

- MAGPIS: The Multi-Array Galactic Plane Imaging Survey

- MI: Meio Interestelar

- MIPS: Multiband Imaging Photometer for the Spitzer

- OTF: On-The-Fly

- PAH: Polycyclic Aromatic Hydrocarbon

- PDR: Photon-Dominated Regions

- PMO: Purple Montain Observatory

- PMS: Pre-Main Sequence

- PWV: Precipitable Water Vapor

- RDI: Radiatively-Driven Implosion

- SED: Spectral Energy Distribution

- UV: Ultraviolet

- WISE: Wide-Field Infrared Survey Explorer satellite

- YSO: Young Stellar Object 


\section{Sumário}

1. Introdução . . . . . . . . . . . . . . . . . 25

$1.1 \mathrm{O}$ meio interestelar $\ldots \ldots \ldots \ldots \ldots \ldots$

1.2 Formação estelar . . . . . . . . . . . . . . . . . . . . 28

1.3 PDR: Regiões Dominadas por Fótons . . . . . . . . . . . . . . . . 31

1.4 Moléculas e grãos interestelares . . . . . . . . . . . . . . 33

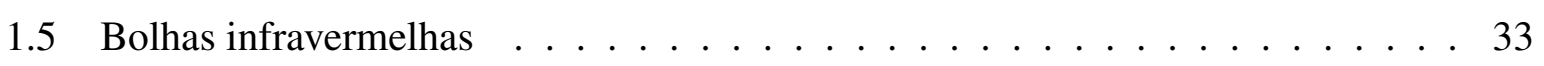

1.6 Formação estelar em bolhas infravermelhas . . . . . . . . . . . . . 35

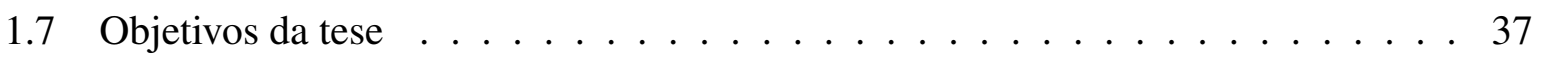

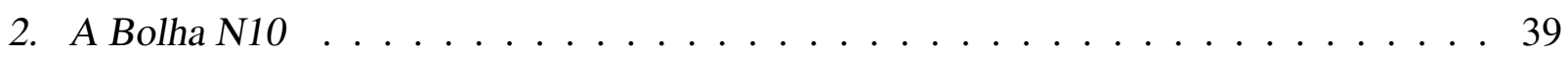

2.1 O nosso objeto de estudo . . . . . . . . . . . . . . . . . 39

2.2 Dados da literatura . . . . . . . . . . . . . . . 43

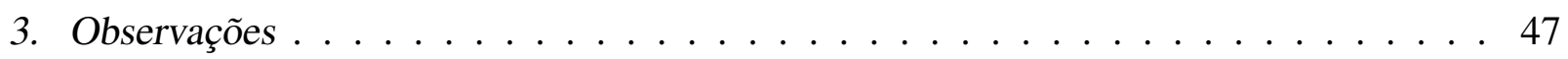

3.1 Observações com o PMO 13.7-m . . . . . . . . . . . . . 4 47

3.2 Observações com o IRAM $30-\mathrm{m} \ldots \ldots \ldots \ldots$

4. Resultados ................................. 51

4.1 Observações com o PMO 13.7-m . . . . . . . . . . . . . . 51

4.2 Distribuição de poeira fria . . . . . . . . . . . . . 61

4.3 Gás ionizado . . . . . . . . . . . . . . . . 63

4.4 Presença de poeira morna na região . . . . . . . . . . . . . 66

4.5 Identificação de YSOs no campo de N10 . . . . . . . . . . . . . . . 67

4.6 Ajustes de SED . . . . . . . . . . . . . . . . 71 
4.7 Resultados para observações no IRAM 30-m . . . . . . . . . . . . . . . 74

4.7 .1 Mapas On-The-Fly . . . . . . . . . . . . . . . 74

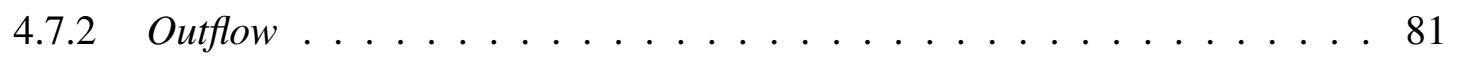

4.7 .3 Integração profunda . . . . . . . . . . . . . 83

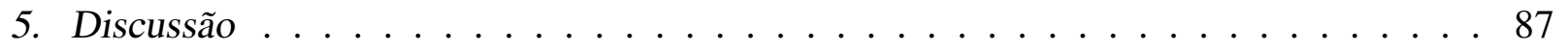

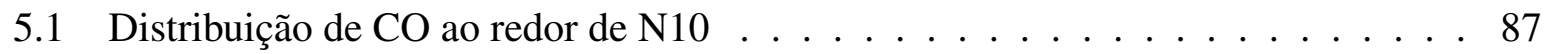

5.1.1 Outras componentes da emissão em CO . . . . . . . . . . . . . 88

5.1 .2 Situação da formação estelar . . . . . . . . . . . . . . . . 89

5.1 .3 A bolha $\mathrm{N} 11 \ldots \ldots \ldots \ldots$

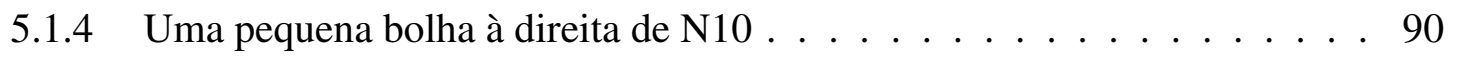

5.2 Traçadores moleculares de formação estelar . . . . . . . . . . . . . . . 91

5.2 .1 Ionização . . . . . . . . . . . . . . . . . . . . . 91

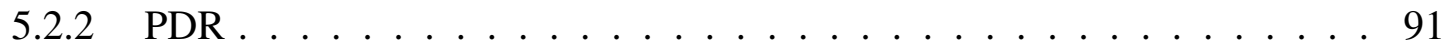

5.2 .3 Choques/Outflows ............................ 92

5.2 .4 Cores pré-estelares .......................... 93

5.2 .5 Gás denso . . . . . . . . . . . . . . . . 93

5.2.6 Levantamento de linhas espectrais . . . . . . . . . . . . 94

6. Conclusões e Perspectivas . . . . . . . . . . . . . . . . 95

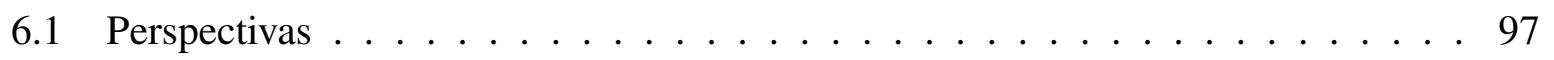

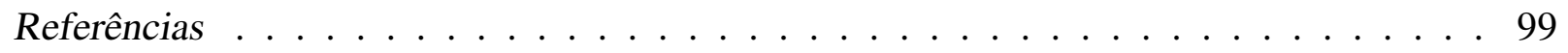

Apêndice 107

A. Lista de YSOs identificados em N10 . . . . . . . . . . . . . . . . . . 109 


\section{Capítulo 1}

\section{Introdução}

“Ora (direis) ouvir estrelas!

Certo, perdeste o senso!"

Olavo Bilac

Ao longo de sua vida, uma estrela afeta profundamente o meio que a circunda: ela pode aquecer o gás ao redor com sua radiação, varrer matéria para longe com seu vento, sintetizar elementos pesados que são a base da vida como a conhecemos e induzir a formação de outras estrelas. A formação estelar desencadeada por meio da interação de estrelas com o seu meio ambiente é um tópico de grande importância na Astrofísica - e o foco desta tese.

\subsection{O meio interestelar}

Para estudar a formação estelar é necessário conhecer o meio interestelar (MI), uma vez que as estrelas se formam a partir do colapso gravitacional de estruturas do MI. O MI é constituinte essencial da nossa Galáxia, pois nele estão átomos, moléculas, íons, grãos de poeira, raios cósmicos, campos magnéticos. O MI não é homogêneo, apresentando uma estrutura que se divide de forma hierárquica: de nuvens moleculares, que abrigam berçários estelares, até condensações densas (cores, em inglês). Uma instabilidade gravitacional, seja ela espontânea ou estimulada externamente, pode gerar o colapso de um core e iniciar o processo de formação estelar.

O MI pode ser separado em fases, que são divididas de acordo com a interação entre os diferentes processos físicos - resfriamento/aquecimento - no ambiente. Dessa forma, cada fase possui temperatura e densidade distintas, como a Tabela 1.1 descreve. 


\begin{tabular}{lcccc}
\multicolumn{5}{c}{ Tabela 1.1 - Fases do MI e suas propriedades físicas. Adaptado de $\mathrm{Kwok}(2007)}$. \\
\hline \hline Componente do MI & $\begin{array}{c}\text { Temperatura } \\
(\mathrm{K})\end{array}$ & $\begin{array}{c}\text { Densidade } \\
\left(\mathrm{cm}^{-3}\right)\end{array}$ & $\begin{array}{c}\text { Estado do } \\
\text { Hidrogênio }\end{array}$ & Traçador típico \\
\hline Gás Coronal & $10^{6}$ & 0.003 & $\mathrm{H}^{+}$ & Raios-X \\
Gás Ionizado Difuso & $10^{4}$ & $>10$ & $\mathrm{H}^{+}$ & Infravermelho \\
HI internuvens & $10^{3}-10^{4}$ & 0.1 & $\mathrm{H}^{0}$ & Emissão em $21 \mathrm{~cm}$ \\
Nuvens difusas & 100 & $10-100$ & $\mathrm{H}^{0}+\mathrm{H}_{2}$ & $3.4 \mu$ m em absorção \\
Nuvens moleculares & 10 & $10^{3}-10^{5}$ & $\mathrm{H}_{2}$ & Moléculas detectadas em rádio \\
& & & & $\left(\mathrm{CO}, \mathrm{CS}, \mathrm{NH}_{3} \ldots\right)$ \\
\hline \hline
\end{tabular}

As estrelas se formam dentro de nuvens moleculares, que abrigam núcleos densos de gás e poeira. A visão clássica da formação estelar no meio interestelar consiste na fragmentação gravitacional (Shu, 1977). Nesse cenário, grandes nuvens moleculares se fragmentam em subregiões chamadas clumps que, por sua vez, se dividem em condensações menores conhecidas como cores. A seguir, descrevemos as características da estrutura hierárquica das nuvens moleculares.

\section{Nuvens}

A matéria que constitui o meio interestelar se agrupa em nuvens, que podem ter características distintas. Por isso, são classificadas como nuvens moleculares, nuvens escuras ou nuvens escuras infravermelhas.

- Nas nuvens moleculares é onde a maior parte do gás molecular da Galáxia se concentra. Abrangem desde pequenas nuvens moleculares (tamanhos de $\sim 2$ pc e massas de $\sim 10^{3} \mathrm{M}_{\odot}$ ) até nuvens moleculares gigantes $\left(\sim 15 \mathrm{pc}\right.$ e $\left.\sim 10^{4} \mathrm{M}_{\odot}\right)$. Nessas nuvens, a temperatura é da ordem de $10 \mathrm{~K}$.

- Já as nuvens escuras correspondem às ditas pequenas nuvens moleculares citadas acima, e são densas o suficiente para absorver a radiação em comprimentos de onda ópticos e ocasionar a extinção visual. Normalmente, estão associadas a regiões de formação de estrelas de baixa massa e massa intermediária.

- As nuvens escuras infravermelhas (IRDC, do inglês Infrared Dark Clouds) foram descobertas recentemente (Perault et al., 1996), e se apresentam como estruturas filamentares e escuras contra um fundo brilhante em infravermelho médio provindo do plano da Galáxia. Essas nuvens supostamente são as progenitoras de estrelas massivas em aglomerados. 
As informações acerca das nuvens moleculares são obtidas, em sua maioria, a partir de estudos da distribuição da molécula de CO na região.

\section{Clumps}

Com a fragmentação hierárquica, nuvens moleculares em menores escalas podem conter subregiões filamentárias, com sobre-densidade, conhecidas como clumps. Dados de $\mathrm{CO}$ e de ${ }^{13} \mathrm{CO}$ fornecem propriedades como massa, dimensão e densidade dessas condensações.

\section{Cores}

Condensações de gás e poeira da ordem de 0.03 - 0.2 pc de dimensão nem sempre abrigam estrelas em seu interior. Nesse caso, são chamados cores sem estrelas (do inglês starless cores). Cores pré-estelares são aqueles que ainda não hospedam estrela em formação. Porém, quando um core pré-estelar torna-se gravitacionalmente instável, uma estrela ou um sistema binário de estrelas é formado. Estes são os chamados cores protoestelares, também conhecidos como objetos Classe 0. Mais adiante, discutiremos a classificação de objetos estelares jovens.

Cores pré-aglomerados, analogamente, formam associações massivas de estrelas dos tipos $\mathrm{O}$ e B. Neste trabalho, entretanto, focaremos em condensações que formam estrelas de baixa massa e massa intermediária, que chamaremos genericamente de cores estelares.

A Tabela 1.2 descreve as características acima mencionadas.

Tabela 1.2 - Propriedades de nuvens moleculares, clumps e cores estelares. (Tabela adaptada de Bergin \& Tafalla 2007).

\begin{tabular}{lccc}
\hline \hline & $\begin{array}{c}\text { Nuvens } \\
\text { Moleculares }\end{array}$ & Clumps & $\begin{array}{c}\text { Cores } \\
\text { Estelares }\end{array}$ \\
\hline Tamanho $(\mathrm{pc})$ & $2-15$ & $0.3-3$ & $0.03-0.2$ \\
Densidade média $\left(\mathrm{cm}^{-3}\right)$ & $50-500$ & $10^{3}-10^{4}$ & $10^{4}-10^{5}$ \\
Massa $\left(\mathrm{M}_{\odot}\right)$ & $10^{3}-10^{4}$ & $50-500$ & $0.5-5$ \\
Temperatura $(\mathrm{K})$ & $\sim 10$ & $10-20$ & $8-12$ \\
\hline \hline
\end{tabular}

As nuvens moleculares são portanto frias e densas. Nessas condições, as transições rotacionais são as mais facilmente excitadas, como nas moléculas $\mathrm{H}_{2}$ e $\mathrm{CO}$, por exemplo. Embora $\mathrm{H}_{2}$ seja a mais abundante, não possui momento de dipolo elétrico permanente, portanto não apresenta linhas rotacionais. Já CO é a segunda molécula mais abundante e tem momento de dipólo 
permanente. Além disso, seu primeiro nível de rotação acima do estado fundamental é de $5 \mathrm{~K}$, que é facilmente excitado.

\subsection{Formação estelar}

A formação de estrelas de baixa massa é bem conhecida, tanto em termos observacionais quanto teóricos (e.g. McKee \& Ostriker, 2007, e suas referências). Por outro lado, a formação das estrelas de alta massa ainda não é tão bem compreendida (Zinnecker \& Yorke, 2007). Estrelas de alta massa são importantes na estrutura e na evolução das galáxias, pois são importantes fontes de calor, turbulência, raios-X e radiação ultravioleta, além de enriquecerem o MI com elementos mais pesados do que $\mathrm{H}$ e He mais rapidamente do que as estrelas de baixa massa.

A dificuldade em se estudar os processo de formação de estrelas massivas reside no fato de que elas são formadas em nuvens de alta densidade bem distantes de nós (> $1 \mathrm{kpc}$ ). Outro obstáculo é o desafio imposto aos modelos teóricos, pois estrelas massivas raramente se formam isoladamente. Além disso, estrelas de alta massa se formam em menores escalas de tempo ( $10^{5}$ anos), o que implica em poucas candidatas para um estudo estatístico na fase mais embebida de sua formação. Por isso, na presente tese, focaremos na formação de estrelas de baixa massa.

\section{Formação de estrelas de baixa massa}

As estrelas são formadas em pontos de instabilidade em nuvens interestelares, quando a pressão interna e a turbulência não podem estabilizar as condensações contra o colapso gravitacional. A descrição do processo de formação estelar se inicia em uma nuvem considerada esférica, que entra em colapso gravitacional em uma escala de tempo caracterizada como "tempo de queda livre" (do inglês free-fall time) definido por Shu et al. (1987) como:

$$
t_{f f} \simeq 2.1 \times 10^{5}\left(\frac{\rho}{10^{-19} \mathrm{~g} \mathrm{~cm}^{-3}}\right)^{-1 / 2} \text { anos }
$$

Um core entra em colapso para formar uma estrela quando sua massa ultrapassa o valor da massa de Jeans (1928), definida como sendo a massa mínima para que a atração gravitacional seja forte o suficiente para contrabalançar a pressão do gás.

$$
M_{J}(r) \simeq 1.1\left[\frac{T(r)}{10 K}\right]^{3 / 2}\left[\frac{\rho(r)}{10^{-19} \mathrm{gcm}^{-3}}\right]^{-1 / 2} M_{\odot}
$$


onde $T(r)$ é a temperatura em função do raio e $\rho(r)$ o perfil de densidade. Quando a massa do core é maior do que a massa de Jeans, ele irá colapsar.

Considerando que os cores tem densidade típica de $\leq 10^{-19} \mathrm{~g} \mathrm{~cm}^{-3}$, o gás cairá na direção do centro da estrela em formação em $t_{f f} \leq 2 \times 10^{5}$ anos.

As estrelas de baixa massa em processo de formação são definidas como objetos estelares jovens (YSO, do inglês Young Stellar Object). Eles são constituídos pelas seguintes componentes: núcleo em colapso, protoestrela, disco de acreção, envoltório e outflow/jato. Veja a Seção 1.4 de Gama, D.R.G. (2012) para maiores detalhes.

Tais objetos são, em sua grande maioria, encontrados embebidos em gás e poeira de nuvens moleculares. Por isso, usamos dados no infravermelho para reduzir os efeitos de extinção na linha de visada. A Figura 1.1 reúne as informações das principais fases da evolução dos objetos estelares jovens.

\begin{tabular}{|c|c|c|c|c|c|}
\hline PROPRIEDADES & $\begin{array}{l}\text { Protoestrela } \\
\text { com material } \\
\text { em queda }\end{array}$ & $\begin{array}{l}\text { Protoestrela } \\
\text { evoluída }\end{array}$ & $\begin{array}{l}\text { Estrela } \\
\text { T Tauri } \\
\text { Clássica }\end{array}$ & $\begin{array}{c}\text { Estrela } \\
\text { T Tauri de } \\
\text { linhas fracas }\end{array}$ & $\begin{array}{l}\text { Estrela na } \\
\text { Sequência } \\
\text { Principal }\end{array}$ \\
\hline ESBOÇO & $\frac{1}{x / 1} d$ & & 17 & $\frac{1}{i}$ & $\circ \circ$ \\
\hline $\begin{array}{l}\text { IDADE } \\
\text { (ANOS) }\end{array}$ & $10^{4}$ & $10^{5}$ & $10^{6}-10^{7}$ & $10^{6}-10^{7}$ & $>10^{7}$ \\
\hline $\begin{array}{l}\text { CLASSE } \\
\text { NO MM / IV }\end{array}$ & Classe 0 & Classe I & Classe II & Classe III & (Classe III) \\
\hline DISCO & Sim & Espesso & Espesso & $\begin{array}{l}\text { Fino ou } \\
\text { Inexistente }\end{array}$ & $\begin{array}{l}\text { Possível } \\
\text { Sistema } \\
\text { Planetário }\end{array}$ \\
\hline RAIOS-X & ? & Sim & Forte & Forte & Fraco \\
\hline $\begin{array}{l}\text { EMISSÃO } \\
\text { TÉRMICA } \\
\text { EM RÁDIO }\end{array}$ & Sim & Sim & Sim & Não & Não \\
\hline $\begin{array}{c}\text { EMISSÃO } \\
\text { NÃO-TÉRMICA } \\
\text { EM RÁDIO }\end{array}$ & Não & Sim & Não? & Sim & Sim \\
\hline
\end{tabular}

Figura 1.1: Esquema dos estágios da evolução de objetos estelares jovens, de acordo com suas denominações no milimétrico/infravermelho. (Feigelson \& Montmerle, 1999).

Portanto, torna-se necessário definir cada classe de YSO. A seguir descrevemos a definição 
desses objetos e na Seção 4.5 relatamos como identificamos os YSOs na direção da bolha N10.

\section{Definição de YSO}

Nessa tese estudamos a distribuição de três tipos de YSOs: Classe I, Classe II e Disco de Transição. De acordo com Strom et al. (1989), Feigelson \& Montmerle (1999), Koenig et al. (2012) e Koenig \& Leisawitz (2014), definimos tais objetos da seguinte forma:

- Classe I são os objetos candidatos a protoestrelas, que ainda têm tanto envoltório quanto disco. São YSOs mais avermelhados com envoltório em queda na direção central. Para esses objetos, a distribuição espectral de energia (ou SED, do inglês Spectral Energy Distribution) é crescente entre 3.3 e $22 \mu \mathrm{m}$. O tempo de vida de um objeto Classe I é de aproximadamente $10^{5}$ anos. A característica de forte absorção em $9.7 \mu$ m é atribuída aos silicatos presentes nos mantos de gelo, que crescem nos grãos de poeira localizados no envoltório (e.g. Chiar et al., 2000, e suas referências).

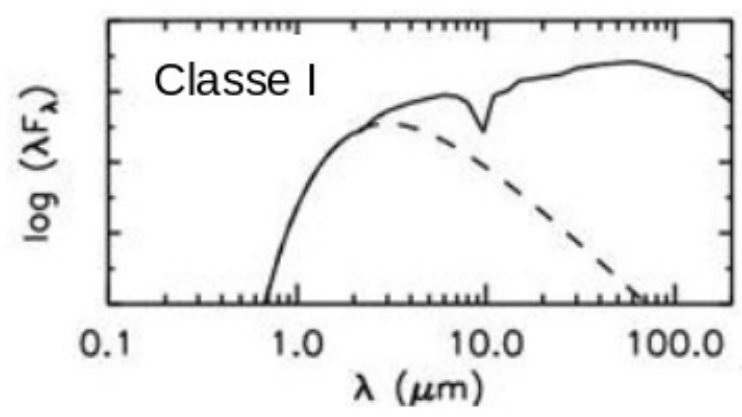

Figura 1.2: Exemplo de SED de um objeto YSO Classe I. A curva em preto representa a emissão total do YSO e a linha tracejada é a curva de corpo negro. A contribuição do envoltório é evidenciada pelo excesso de emissão no infravermelho distante. (Figura adaptada de Wilking, 1989).

- Classe II são estrelas pré-sequência principal (ou PMS, do inglês Pre-Main Sequence) com disco opticamente espesso, mas sem envoltório significativo, candidatas a estrelas do tipo T Tauri e Herbig AeBe. A SED dos YSOs Classe II tem inclinação decrescente ou plana entre o infravermelho próximo e médio. O tempo de vida de um objeto Classe II é de aproximadamente $3 \times 10^{6}$ anos, embora haja um grande intervalo de idades estimadas para esse tipo de objeto, variando de $1-10 \times 10^{6}$ anos. 


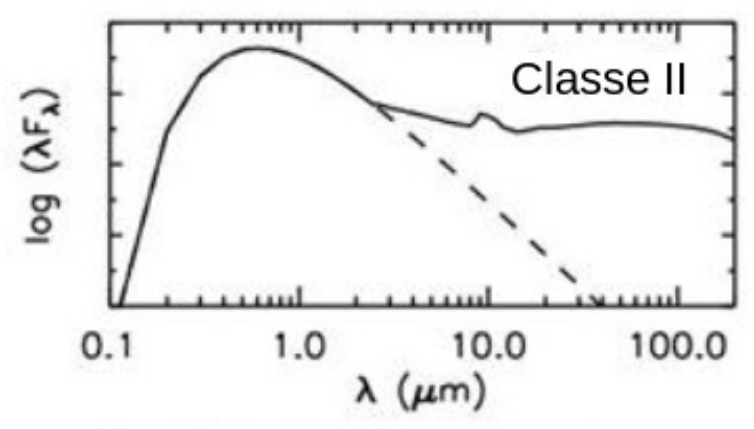

Figura 1.3: Exemplo de SED de um objeto estelar jovem Classe II. (Figura adaptada de Wilking, 1989).

- Disco de transição é uma subclasse de estrelas jovens com uma lacuna na parte interna de seus discos, emitindo níveis reduzidos de infravermelho próximo e/ou excesso no infravermelho médio (especialmente em $22 \mu \mathrm{m}$; Koenig \& Leisawitz, 2014). Embora a região interior do disco seja relativamente desprovida de matéria, a regiões mais externas contêm quantidades consideráveis de poeira (Strom et al., 1989).

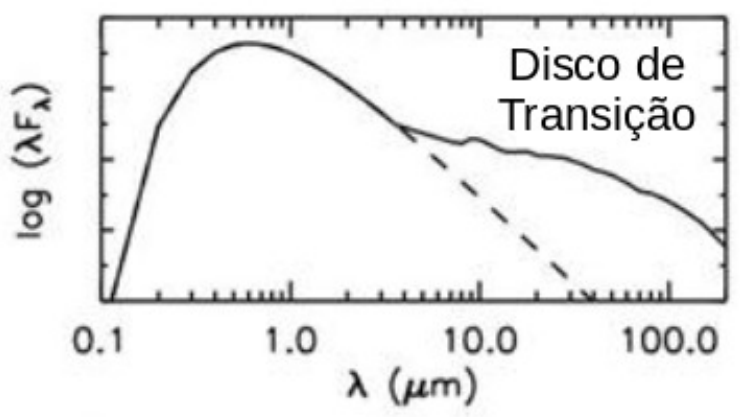

Figura 1.4: Exemplo de SED de um Disco de Transição. A distribuição se assemelha a um corpo negro estelar, atingindo seu máximo em comprimentos de onda ópticos ou infravermelhos. Excesso no infravermelho médio ainda é muitas vezes observado, possivelmente devido ao disco fino restante. (Figura adaptada de Wilking, 1989).

\subsection{PDR: Regiões Dominadas por Fótons}

A maior parte das alterações térmicas nas nuvens moleculares ocorrem perto de estrelas. As chamadas PDRs (Regiões Dominadas por Fótons, do inglês Photon-Dominated Regions ou Photodissociation Regions) são regiões ao redor de estrelas dos tipos $\mathrm{O}$ e $\mathrm{B}$ em que os fótons UV (ultravioleta), com energia $6 \mathrm{eV}<h v<13.6 \mathrm{eV}$, dominam o balanço de energia do gás. 
PDRs são associadas a regiões de alta densidade e alto fluxo FUV (ultavioleta distante, do inglês far-ultraviolet).

Os fótons FUV penetram a nuvem dissociando, ionizando e aquecendo o gás. Fótons com energia menor que $13.6 \mathrm{eV}$ dissociam o hidrogênio molecular e ionizam o carbono, formando uma região HI. Mais profundamente na nuvem, quando a extinção visual atinge um valor de $A_{V}$ $\simeq 4$ magnitudes, o fluxo cai o suficiente para que o carbono ionizado se recombine e a formação de monóxido de carbono (CO) ocorra. A transição do oxigênio de atômico para molecular ocorre nas fronteiras da PDR. A Figura 1.5 evidencia essa estratificação química.

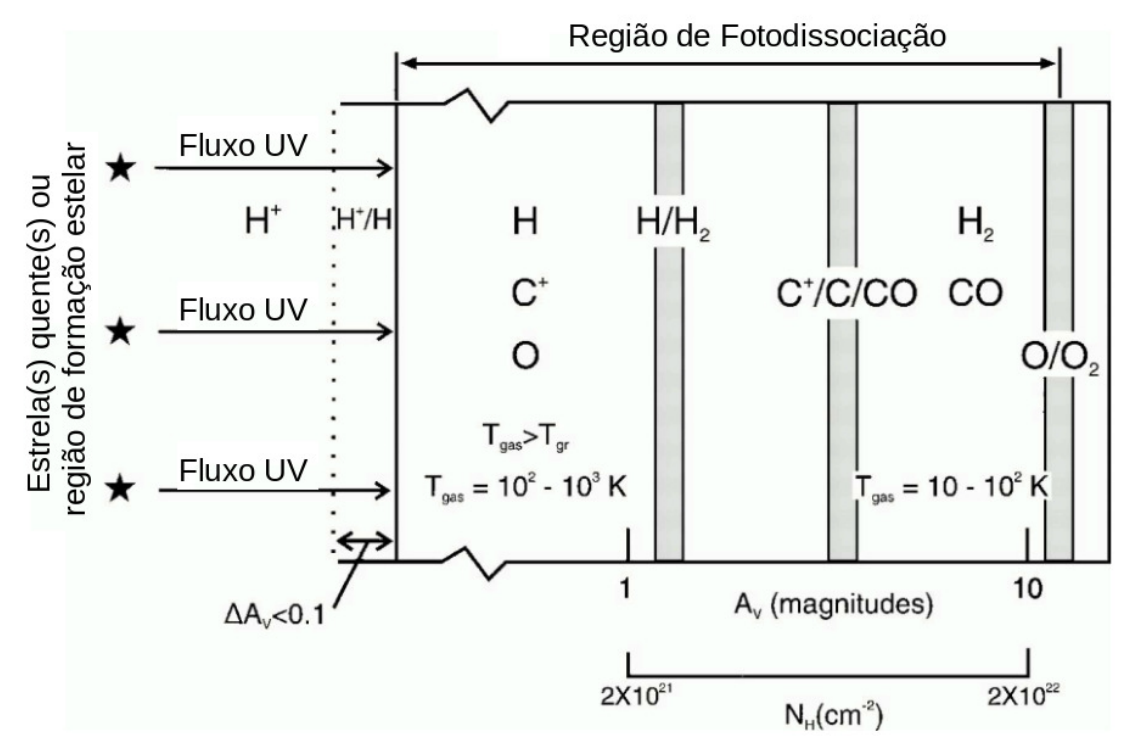

Figura 1.5: Esquema de uma PDR iluminada por um forte campo de radiação pela esquerda. A PDR se extende desde a região de transição $\mathrm{H}^{+} / \mathrm{H}$, passando pelas transições $\mathrm{H} / \mathrm{H}_{2}$ e $\mathrm{C}^{+} / \mathrm{C} / \mathrm{CO}$ até a fronteira $\mathrm{O} / \mathrm{O}_{2}$. (Imagem adaptada de Hollenbach \& Tielens (1999).)

Diversos processos são responsáveis pelo balanço de energia em uma PDR, incluindo o efeito fotoelétrico em grãos e em Hidrocarbonetos Aromáticos Policíclicos (PAHs, do inglês Polycyclic Aromatic Hydrocarbon). O fóton UV pode arrancar elétron do grão, que irá aquecer o gás por meio de colisões.

A química das regiões dominadas por fótons é similar à quimica de nuvens moleculares difusas. Entretanto, nas PDRs os fótons FUV dominam os processos de aquecimento, ionização e química da região ao redor de estrelas quentes e massivas, tornando-os laboratórios astronômicos ideais para o estudo da interação entre fótons FUV e a matéria neutra. 


\subsection{Moléculas e grãos interestelares}

A detecção de moléculas no meio interestelar tem importantes implicações. As medidas da intensidade de suas linhas são ferramentas de diagnóstico eficazes na análise das condições físicas de nuvens moleculares. Em regiões de formação estelar, as moléculas são formadas e destruídas, de acordo com o processo físico em ação. Discutiremos tais processos com maiores detalhes mais adiante.

Regiões neutras do MI podem sofrer o aquecimento devido ao efeito fotoelétrico. Este efeito age sobre grandes moléculas de PAH e sobre grãos de poeira, que absorvem fótons energéticos UV.

Além disso, os grãos atuam como uma superfície sobre a qual algumas moléculas podem se formar (Tielens \& Hagen, 1982). As espécies acretadas se encontram e cedem energia de reação em excesso. Nesse processo, as espécies: se acumulam na superfície dos grãos, se difundem pelo manto de gelo que cobre o grão de poeira, reagem formando uma molécula e evaporam quando a energia liberada pela formação é suficiente para ejetar a molécula da superfície.

\subsection{Bolhas infravermelhas}

Os estudos de formação estelar vem ganhando destaque considerável nas últimas décadas. A possibilidade de que a interação das estrelas massivas com as nuvens moleculares que as envolvem desencadeie a formação estelar é uma questão amplamente discutida. Instrumentos que operam no infravermelho têm ampliado os horizontes nesse campo (e.g. Zavagno et al., 2010). O levantamento GLIMPSE ${ }^{1}$, realizado com a utilização do Spitzer ${ }^{2}$ para obter imagens em comprimentos de onda de 3.6 a $8.0 \mu \mathrm{m}$, revelou uma nova classe de objetos: as bolhas infravermelhas, primeiramente catalogadas por Churchwell et al. (2006). Churchwell et al. (2007) completaram o levantamento de bolhas infravermelhas.

Bolhas infravermelhas são regiões ideais para investigarmos o impacto da radiação ionizante e do forte campo UV sobre as condições de formação estelar nas fronteiras de regiões HII. Esses objetos apresentam uma borda brilhante em $8.0 \mu \mathrm{m}$, causada pela emissão de PAHs excitados pela radiação UV, que envolve uma região de gás ionizado (Churchwell et al., 2006, 2007), como mostra a Figura 1.6

\footnotetext{
${ }^{1}$ Galactic Legacy Infrared Midplane Extraordinaire: http://www.astro.wisc.edu/glimpse/

${ }^{2}$ Spitzer Space Telescope: http://www.spitzer.caltech.edu/
} 


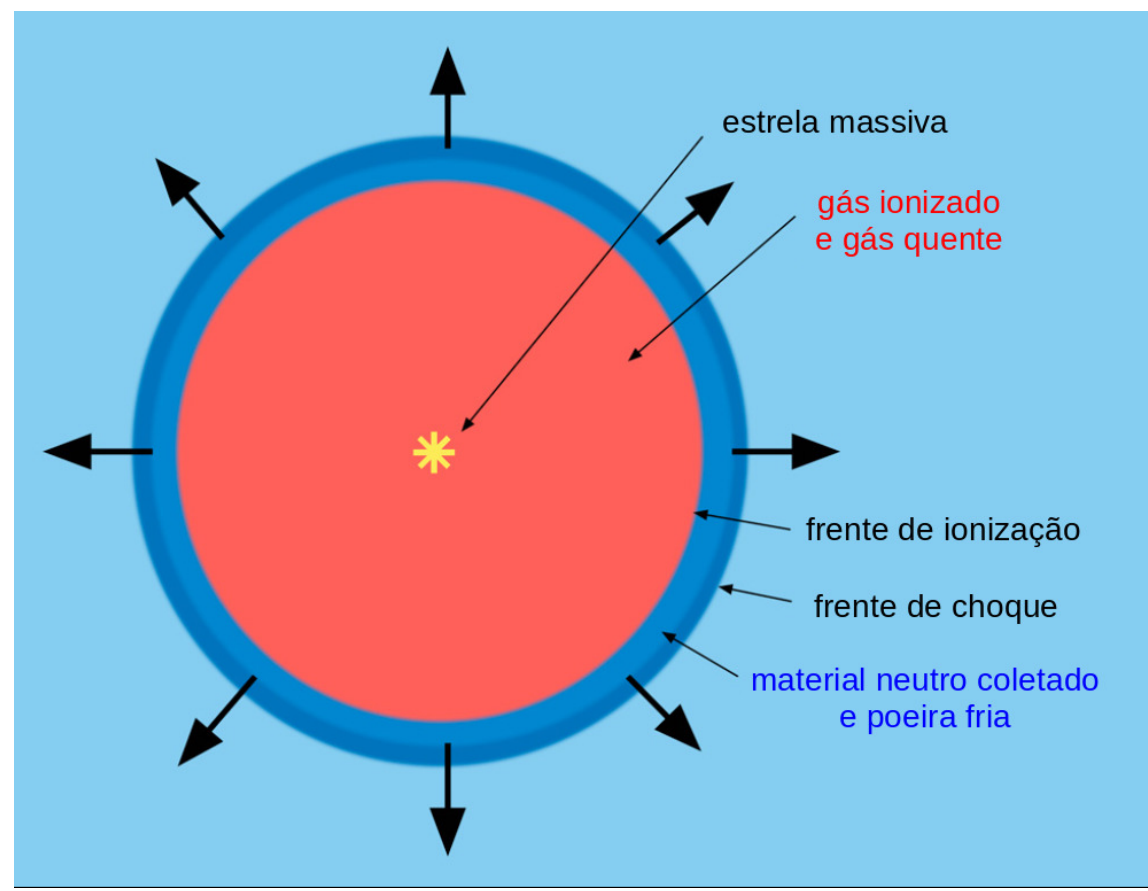

Figura 1.6: Esquema de uma região HII simétrica em expansão, inserida em um meio homogêneo. Nesse modelo, a região HII é esférica e sua porção ionizada é rodeada pelo material denso e neutro, que foi coletado durante a fase de expansão. (Figura adaptada de Deharveng et al., 2010).

Deharveng et al.(2010) identificaram e estudaram uma grande amostra de bolhas, concluindo que a casca de cada uma, detectada a $8.0 \mu \mathrm{m}$, é uma evidência de uma região HII produzida por estrelas ionizantes de alta massa. Cascas densas ao redor de muitas bolhas infravermelhas parecem favorecer a formação de novas gerações de estrelas (Cappa et al., 2016). Estudos estatísticos levaram Thompson et al. (2012) a sugerir que a expansão das bolhas desencadeia a formação de objetos estelares jovens e esse processo é considável em escalas Galácticas. Autores como Kendrew et al. (2012) também observaram esse comportamento e encontraram grande concentração de YSOs nas bordas de bolhas em expansão, que podem oferecer importantes pistas sobre o processo de formação estelar e a dinâmica de bolhas infravermelhas.

Podemos interpretar essas bolhas basicamente como gás ionizado envolvido por poeira fria. Ao redor há uma PDR que pode ser estudada também no infravermelho médio (Lefloch et al. 2005). As estrelas de alta massa nesse lugar interagem com a nuvem molecular original e, através da sua radiação UV, geram uma interface entre o gás ionizado (próximo às estrelas quentes) e o gás neutro (longe das estrelas quentes). Portanto, a densidade de gás é maior na casca destas bolhas, onde os fótons UV penetram com menos energia. 


\subsection{Formação estelar em bolhas infravermelhas}

Tem sido sugerido há tempos que a frente de choque dentro da bolha pode desencadear a formação estelar dentro do arco brilhante dessa bolha (e.g. Reipurth, 1983; Lefloch et al., 1997).

De acordo com Anderson et al. (2015) a morfologia anelar é comum para regiões HII da Galáxia, mas a geometria esférica ou elipsoidal de tais objetos permanece incerta. O estudo da estrutura desses objetos, sejam cascas esféricas ou anéis, nos permite compreender corretamente a cinemática do gás e a sequência de idades das novas estrelas formadas relacionadas com eles. A interação entre estrelas jovens com o gás molecular ao redor da nuvem progenitora e a possibilidade de formação estelar desencadeada tem estimulado um grande número de trabalhos, tanto observacionais quanto teóricos.

Diversos mecanismos de formação estelar desencadeada têm sido propostos (veja e.g. Deharveng et al., 2008; Samal et al., 2014, para uma revisão), entre os quais o cenário “Collect and Colapse" (representados nas ilustrações 1 e 2 na Figura 1.7), inicialmente proposto por Elmegreen \& Lada (1977), tem especial relevância. Nesse cenário, a radiação de estrelas de alta massa de uma região HII cria uma frente de ionização na interface com a nuvem molecular, que conduz a propagação de uma frente de choque para dentro do material molecular. Esse material acumula massa em larga escala e, eventualmente, torna-se gravitacionalmente instável com a expansão da região HII. O cenário "Collect and Colapse" tem sido revisitado desde então (e.g. Whitworth \& Francis, 2002). A região HII Sh104 mostrou ser um caso muito convincente e ilustrativo desse cenário por Deharveng et al. (2003).

Outros cenários de formação estelar desencadeada também são propostos, como por exemplo o modelo "Radiation-Driven Implosion" (RDI - ilustração 4 da Figura 1.7). Esses cenários são baseados na sobrepressão exercida pela frente de ionização, na escala de condensações moleculares na borda ou dentro da região HII (veja também Lefloch \& Lazareff, 1994).

Diversos trabalhos alegam que há evidência de formação estelar desencadeada pela expansão de regiões HII (Dewangan et al., 2012; Zavagno et al., 2010; Beuther et al., 2011). Contudo, trabalhos recentes como os de Tackenberg et al. (2012), Samal et al. (2014) and Liu et al. (2015) enfatizam que a análise de formação estelar engatilhada é complexa. Eles não encontraram evidências claras de colapso de condensações de nuvens desencadeado pela expansão da bolha, e concluíram que a situação ainda não é bem definida. Yuan et al. (2014) descobriram na bolha N6 diferenças da ordem de $30 \mathrm{~km} \mathrm{~s}^{-1}$ entre as velocidades das diferentes partes do anel proje- 
tado, o que pode indicar uma velocidade de expansão muito maior do que aquela considerada por outros autores, de poucos $\mathrm{km} \mathrm{s}^{-1}$ (e.g. Beaumont \& Williams, 2010). Portanto, é importante reunir resultados para um número de bolhas bem estudadas para estabelecer se estas tem histórias de formação semelhantes, e se podem fornecer respostas similares para as questões de sua morfologia e formação estelar desencadeada.

Observacionalmente, mais estrelas luminosas e massivas são encontradas na borda de regiões HII (Dobashi et al., 2002). O cenário "Collect and Colapse” prevê a formação de estrelas mais massivas (tipos O e B). Esta é uma questão importante, já que as estrelas são responsáveis pelo campo UV Galáctico e, consequentemente, pelo balanço de energia e a evolução do gás molecular (PDR) na Galáxia. Deharveng et al.(2010) estudaram a formação estelar na borda de algumas regiões HII envolvidas por bolhas infravermelhas. Eles discutiram a possível relação entre 102 dessas bolhas com as regiões HII que envolvem (i.e. quase todas as bolhas), procurando por pistas sobre a formação estelar desencadeada.

Estudos multi comprimentos de onda detalhados têm sido publicados para um número dessas

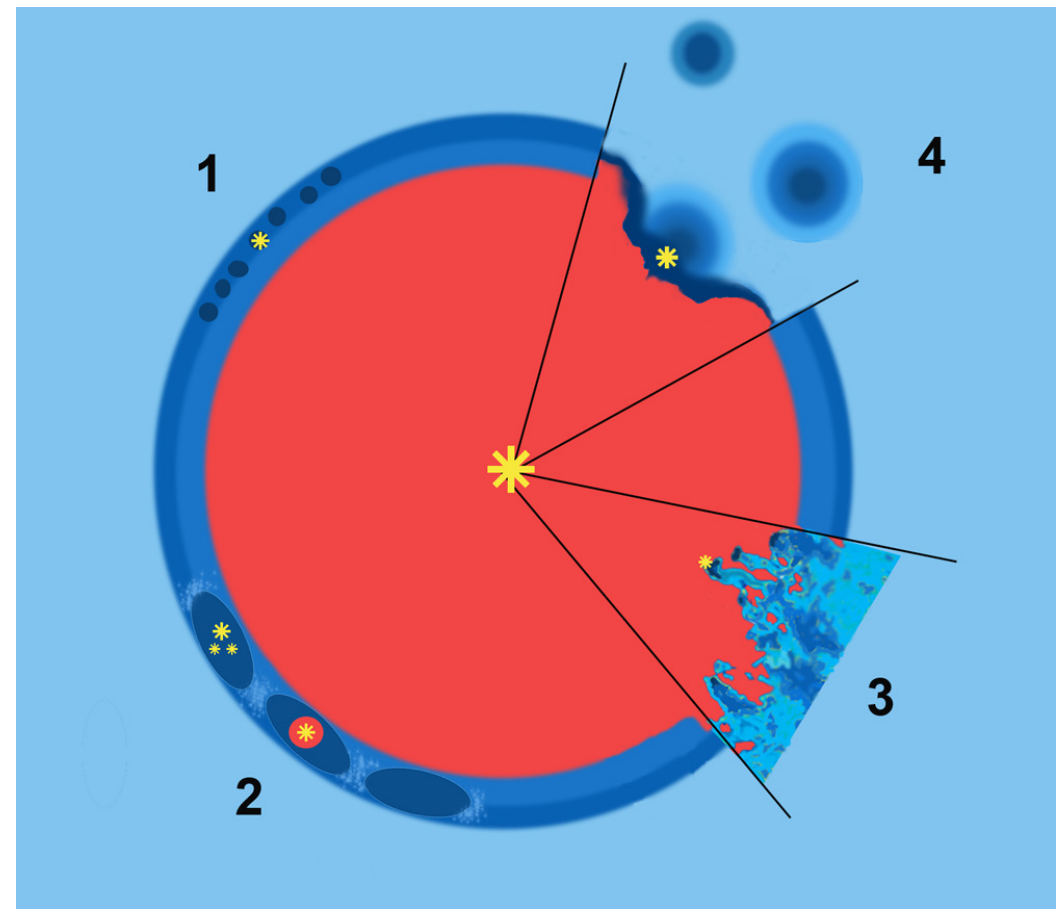

Figura 1.7: Perspectiva esquemática de uma região HII esférica em expansão e ambiente neutro ao redor. Diversos processos de formação estelar desencadeada podem ser caracterizados: 1 - instabilidades gravitacionais em pequena escala; 2 - instabilidades gravitacionais em larga escala, levando à formação de fragmentos de alta massa; 3 radiação ionizante agindo no meio turbulento; 4 - compressão de clumps densos pré-existentes, conduzida por radiação (Figura de Deharveng et al., 2010). 
bolhas, como por exemplo RCW 120 (Zavagno et al., 2010), CN 148 (Dewangan et al., 2015) e N6 (Yuan et al., 2014). Embora os trabalhos supracitados apontem para a presença de YSOs Classe I associados a clumps densos de gás molecular em contato com a região dominada por fótons $(P D R)$, a questão da existência de formação estelar desencadeada associada com a expansão da região HII ainda não está encerrada. No presente trabalho, o nosso principal objetivo é conduzir esse tipo de investigação na bolha infravermelha N10.

\subsection{Objetivos da tese}

O objetivo desta tese é conduzir um estudo detalhado de N10, uma bolha notável que possui condensações moleculares e objetos estelares associados com o ambiente ao redor. Para isso, propomos investigar as propriedades do gás molecular nas bordas da região HII em relação à atividade de formação estelar na região da bolha N10.

No Capítulo 2 apresentamos o nosso objeto de estudo. No Capítulo 3 descrevemos as observações realizadas com os telescópios PMO 13.7-m e IRAM 30-m. Os resultados dessas observações e da análise do objeto também em outros comprimentos de onda estão descritos no Capítulo 4. No Capítulo 5 discutimos os resultados obtidos. As conclusões são listadas no Capítulo 6, que também apresenta as perspectivas de nosso trabalho.

Observação: Parte deste trabalho encontra-se em processo de submissão para publicação em revista científica. Entretanto, todos os resultados (incluindo figuras e gráficos) são exclusivos dessa pesquisa e não foram publicados anteriormente. 


\section{Capítulo 2}

\section{A Bolha N10}

\subsection{O nosso objeto de estudo}

A bolha infravermelha N10 é especialmente interessante por apresentar um cenário de formação estelar sequencial - possivelmente desencadeada - na borda da região HII. Nós utilizamos observações em rádio frequência para investigar as propriedades física e a cinemática do gás, por meio do mapeamento da emissão de traçadores moleculares específicos. Assim podemos caracterizar as propriedades das condensações pré/protoestelares, bem como as propriedades da PDR na interface entre a região HII e as condensações moleculares. Colocando todos esses resultados juntos, podemos ter uma visão detalhada da interação entre a radiação da fonte ionizante e o gás molecular, sua possível função na formação estelar desencadeada e sua influência na evolução química do gás molecular.

A bolha N10, também identificada como MWP1G013189+000428 por Simpson et al. (2012), está situada na direção $1=13.188^{\circ}, \mathrm{b}=0.039^{\circ}$ em coordenadas Galácticas, ou seja, $\alpha_{2000}=$ 18:14:01.36, $\delta_{2000}=-17: 28: 23$ em coordenadas equatoriais (Churchwell et al., 2006). Essa bolha aparece nas imagens em $8.0 \mu \mathrm{m}$ do Spitzer-GLIMPSE como uma estrutura em formato anelar (Figura 2.1). A emissão a $8.0 \mu \mathrm{m}$ na borda das bolhas é atribuída ao PDR contendo PAHs; a densidade do gás na PDR pode ser muito maior (até um fator 10) do que a do meio que a rodeia (Deharveng et al., 2010; Churchwell et al., 2006). Na Figura 2.1 é mostrada uma elipse para demarcar as fronteiras da bolha, para referências futuras em imagens em diferentes comprimentos de onda.

A distância de N10 foi estimada em $4.9 \mathrm{kpc}$ por Churchwell et al. (2006), $4.1 \mathrm{kpc}$ por Beaumont \& Williams (2010), $4.6 \mathrm{kpc}$ por Pandian et al. (2008) e $4.9 \mathrm{kpc}$ por Watson et al. (2008). Essas são distâncias cinemáticas estimadas utilizando diferentes curvas de rotação, o que explica 


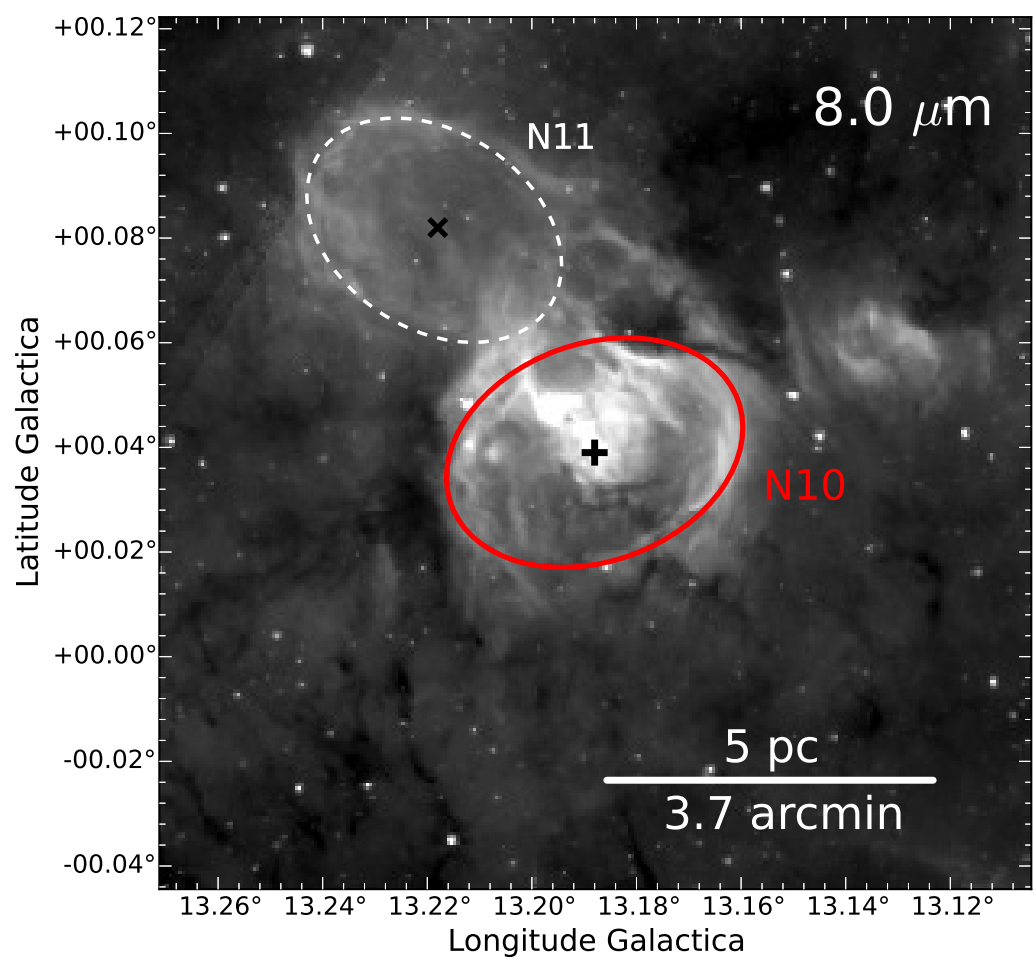

Figura 2.1: Imagem de N10 em $8.0 \mu \mathrm{m}$, localizada a uma distância de $4.7 \mathrm{kpc}$. A cruz preta indica o centro da região HII dentro da bolha infravermelha. A elipse vermelha indica a borda de N10. A escala corresponde a um campo de 5 pc ( 3.7 arcmin). A elipse branca pontilhada indica a posição de N11, cujo centro está localizado em $l=13.218^{\circ}, b=0.082^{\circ}$, aqui identificado com um "x"em preto.

a discrepância. Churchwell et al. (2006) chegaram a considerar que N10 seria uma bolha bipolar (ou dupla), uma vez que uma outra bolha (N11) parece estar conectada a N10. Entretanto, Deharveng et al. (2015) mostraram recentemente que N10/N11 não constituem uma bolha bipolar. Nessa tese lidamos apenas com o caso de N10, mas essa questão será abordada brevemente mais adiante.

A presença do maser de metanol $\left(\mathrm{CH}_{3} \mathrm{OH}\right)$ indica que há impacto de vento no gás denso. Esse processo necessita da radiação de uma estrela jovem e quente para ser energizado (Stahler \& Palla, 2005) e também está associada com estágios iniciais da formação de estrelas de alta massa (Minier \& Booth, 2002). Lee et al. (2001) sugeriram que a emissão maser do metanol a 6.67-GHz em geral está associada com cores moleculares quentes (hot cores). Um maser de metanol na região de N10 foi relatado primeiramente por Szymczak et al. (2000), detectado na direção da fonte IRAS 18111-1729. A Figura 2.2 mostra essa fonte de maser de metanol na extremidade de uma das duas condensações brilhantes a $870 \mu \mathrm{m}$ adjacentes à bolha. $\mathrm{O}$ segundo maser $\mathrm{CH}_{3} \mathrm{OH}$ foi identificado por Pandian et al. (2008), e aparece associado com uma fonte 
SVSS1

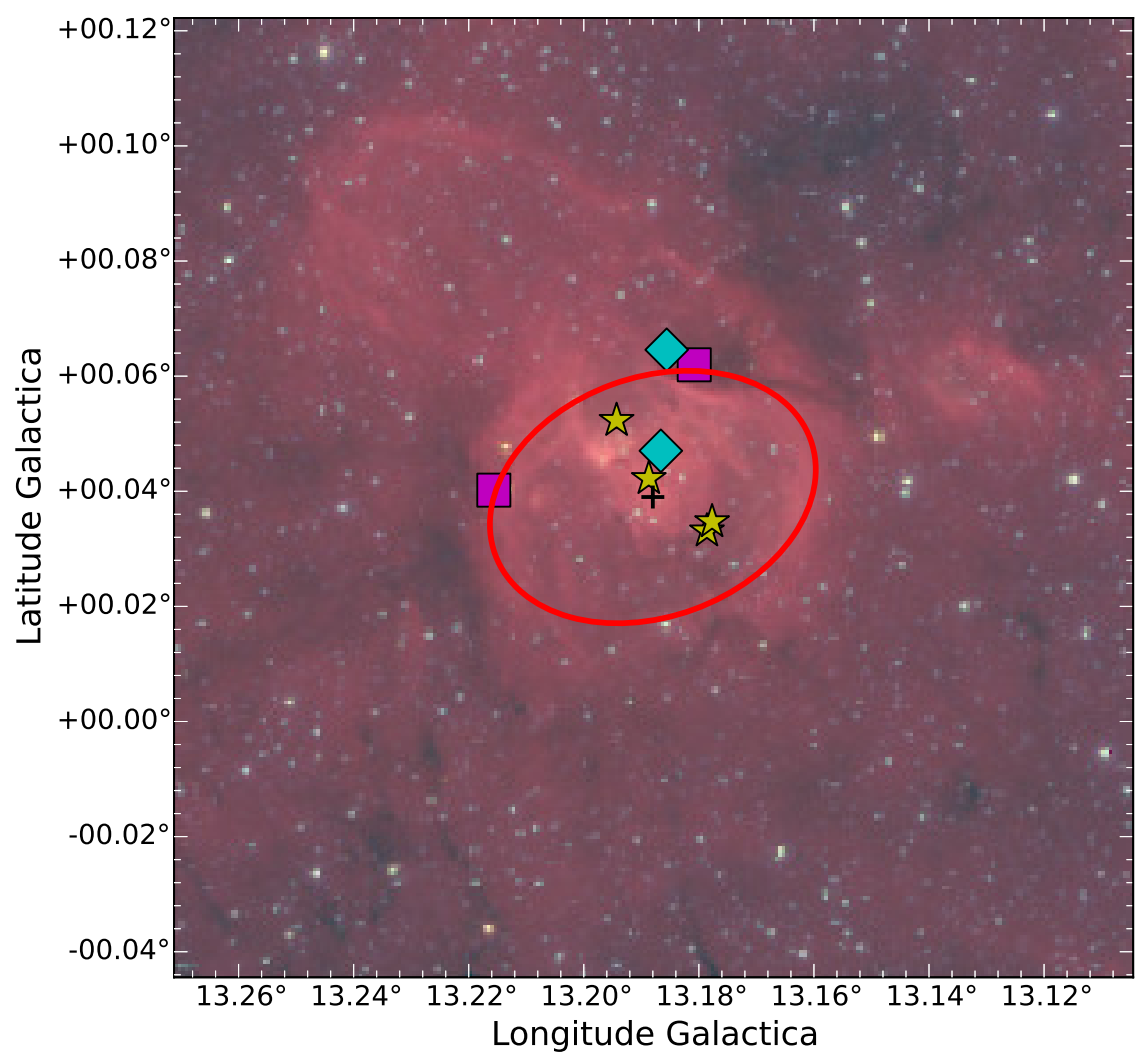

Figura 2.2: Composição em três cores da bolha N10 com imagens Spitzer-GLIMPSE $3.6 \mu \mathrm{m}$ (em azul), $4.5 \mu \mathrm{m}$ (em verde) e $8.0 \mu \mathrm{m}$ (em vermelho). A posição das estrelas identificadas por Watson et al. (2008) estão marcadas em estrelas amarelas; condensações de poeira identificadas por Deharveng et al.(2010) são identificadas por quadrados magenta (\#1 ao lado direito, \#2 ao lado esquerdo); diamantes em ciano representam a posição dos masers de metanol por Szymczak et al. (2000) acima e Pandian et al. (2008) abaixo.

A existência de uma região HII preenchendo a bolha é comprovada pela emissão VLA a $20 \mathrm{~cm}$, em vermelho na Figura 2.2. Em um estudo da região central, Watson et al. (2008) identificaram quatro estrelas como possíveis fontes ionizantes (veja a Tabela 2.1), baseada em suas SEDs, que são bem ajustadas por uma fotosfera estelar.

Um primeiro estudo de baixa resolução angular da emissão de CO associada com N10 foi obtido com o telescópio $\mathrm{PMO}^{2} 13.7-\mathrm{m}$ (Gama et al., 2016). Observamos a transição $J=1-0$ dos isótopos ${ }^{12} \mathrm{CO}(115 \mathrm{GHz}),{ }^{13} \mathrm{CO}(110 \mathrm{GHz})$ e $\mathrm{C}^{18} \mathrm{O}(109 \mathrm{GHz})$. Os resultados são apresentados no Capitulo 4 desta tese.

A resolução angular limitada nas observações realizadas no PMO 13.7-m ( 50 arcsec, ou seja, 1 pc na distância de N10), impede estudos mais detalhados da distribuição do gás molecular

\footnotetext{
${ }^{1}$ NRAO VLA Sky Survey (Condon et al. $[1998$ )

2 Purple Montain Observatory (Nanjing, China)
} 
Tabela 2.1 - Estrelas candidatas a fontes ionizantes no sistema da bolha N10.

\begin{tabular}{ccccc}
\hline \hline ID $^{a}$ & A.R. (J2000) & DEC (J2000) & Tipo Espectral & $\mathrm{A}_{V}$ \\
\hline IN10-1 & 181406.343 & -172833.86 & O7.5 V & 7 \\
IN10-2 & 181404.771 & -172758.74 & O6.5 V & 7 \\
IN10-3 & 181407.104 & -172921.27 & O6 V & 5 \\
IN10-4 & 181406.666 & -172921.34 & O7 V & 8 \\
\hline
\end{tabular}

Notas. ${ }^{a}$ Identificações de Watson et al. (2008).

e sua cinemática e das propriedades do gás na interface entre a região HII e a nuvem molecular progenitora, além da identificação de condensações densas pré/protoestelares.

Nos últimos anos estamos obtendo progresso na compreensão dessa questão, em função de instrumentos como o IRAM ${ }^{3} 30-\mathrm{m}$, que tem possibilitado um levantamento mais completo de traçadores de formação estelar em regiões similares ao nosso objeto de estudo. Dessa forma, recorremos a observações com este telescópio para obter dados mais aprofundados.

A partir daqui adotaremos a posição de N10 e da elipse na Figura 2.2 como referência: topo da bolha (latitudes mais altas com respeito ao centro), inferior (latitudes mais baixas), inferior (longitudes mais baixas) e esquerda (longitudes mais altas).

\footnotetext{
${ }^{3}$ http://www.iram-institute.org/EN/30-meter-telescope.php
} 


\subsection{Dados da literatura}

No presente trabalho, utilizamos levantamentos disponíveis desde o infravermelho até o centimétrico para analisar a bolha N10 em outros comprimentos de onda.

- Infravermelho médio:

O levantamento GLIMPSE (Benjamin et al., 2003) mapeou regiões do plano interno da Galáxia com o instrumento IRAC (Infrared Array Camera; Fazio et al., 2004), a bordo do Telescópio Espacial Spitzer. Nós obtivemos imagens nas bandas IRAC em 4.5, 5.8 and $8.0 \mu \mathrm{m}$, conforme mostra a Figura 2.3 .

Para analisar o conteúdo estelar jovem, nós fizemos uso dos dados Wide-Field Infrared Survey Explorer satellite (WISE; Wright et al., 2010) em 3.4, 4.6, 12, e $22 \mu \mathrm{m}$ (bandas w1, w2, w3, w4 respectivamente). Dessa forma, fomos capazes de identificar regiões nas quais objetos estelares jovens estão presentes.

A imagem de $\mathrm{N} 10$ em $24 \mu \mathrm{m}$ foi obtida por outro levantamento na parte interna do plano Galáctico, MIPSGAL, utilizando o instrumento MIPS (Multiband Imaging Photometer for the Spitzer; Rieke et al., 2004). No painel em $24 \mu \mathrm{m}$ da Figura 2.3 podemos ver que essa emissão preenche toda a área indicada pela elipse vermelha. Essa emissão, típica em bolhas infravermelhas, é causada pela poeira morna presente na região do gás ionizado.

- Sub-milimétrico:

A bolha N10 foi mapeada em comprimentos de onda no sub-milimétrico pelo telescópio APEX (Miettinen, 2012). A imagem em $870 \mu \mathrm{m}$ foi obtida com o ATLASGAL (APEX Telescope Large Area Survey of the Galaxy), um programa de observação que utilizou o intrumento LABOCA (Large Apex BOlometer CAmera instrument Schuller et al., 2009). A emissão da poeira fria em $870 \mu \mathrm{m}$ é útil para revelar a presença de nuvens escuras densas. Duas dessas nuvens, relatadas por Wienen et al. (2012), são vistas nas fronteiras de N10.

\section{- Centimétrico:}

A emissão no contínuo de rádio em $20 \mathrm{~cm}$ a partir dos dados MAGPIS (Helfand et al. 2006) confirma a existência de uma região HII dentro da bolha N10. Com o intuito de melhorar

\footnotetext{
4 The Multi-Array Galactic Plane Imaging Survey
} 
os dados da emissão no contínuo, o MAGPIS combinou imagens VLA com imagens de um levantamento em $1.4 \mathrm{GHz}$ realizado por Reich et al. (1990) utilizando o telescópio Effelsberg 100-m. Tal emissão, devido ao processo livre-livre, é boa traçadora de gás ionizado.

Nós usamos o website MAGPIS 5 para obter as imagens apresentadas na Figura 2.3.

\footnotetext{
${ }^{5}$ http://third.ucllnl.org/gps
} 

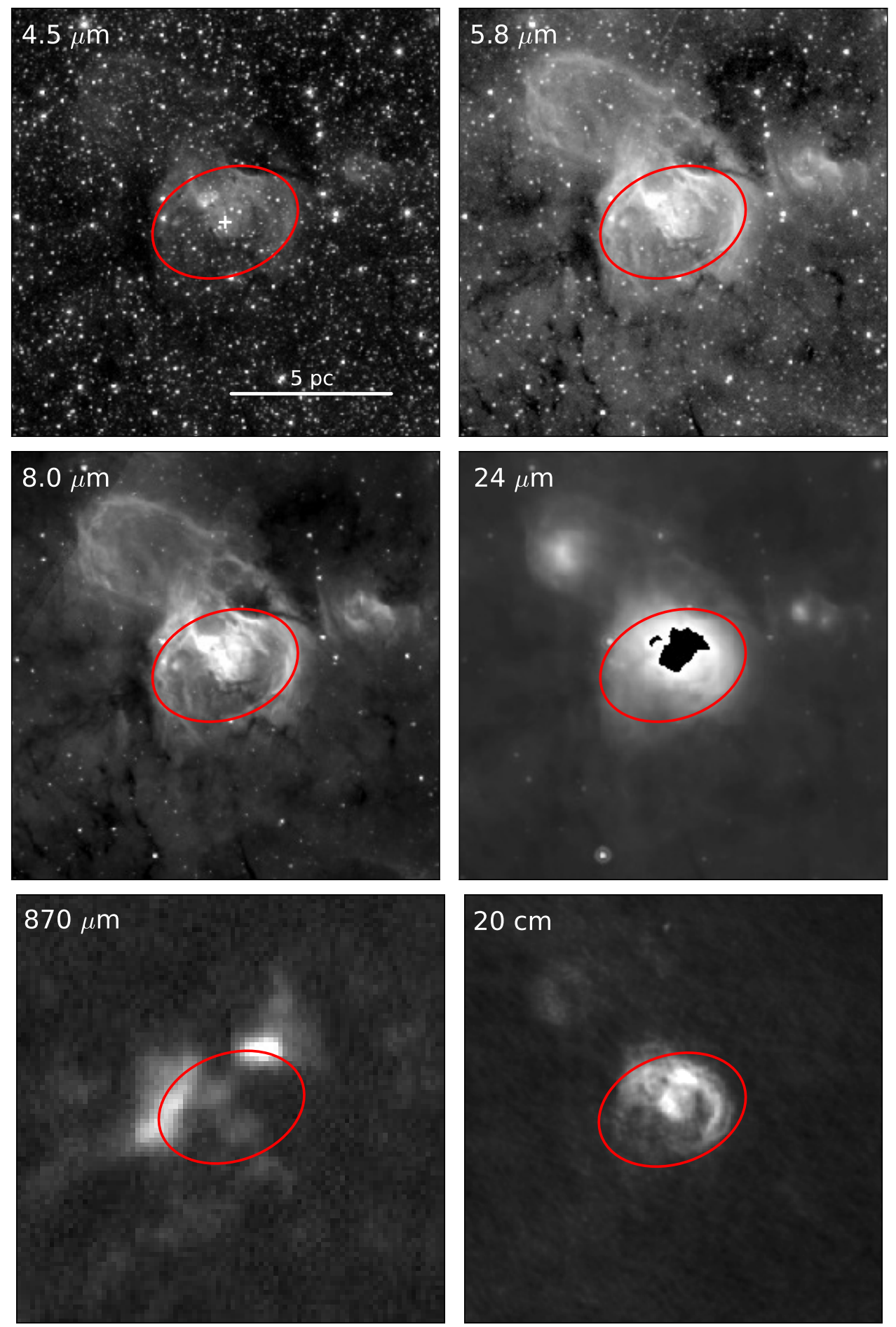

Figura 2.3: Imagens multi-comprimento de onda da região N10. Os painéis mostram a emissão em $4.5 \mu \mathrm{m}, 5.8$ $\mu \mathrm{m}, 8.0 \mu \mathrm{m}, 24 \mu \mathrm{m}, 870 \mu \mathrm{m}$ e $20 \mathrm{~cm}$ na direção da região de interesse, dos levantamentos Spitzer-GLIMPSE, Spitzer-MIPSGAL, APEX/ATLASGAL e GPS-VLA, respectivamente. Todas as imagens mostram a mesma região do céu, no primeiro painel, a cruz branca marca o centro da região HII; em todas as imagens a elipse vermelha é para referência, conforme mostrado na Figura 2.1 . 


\section{Capítulo 3}

\section{Observações}

\subsection{Observações com o PMO 13.7-m}

PMO 13.7-m é uma antena que encontra-se no Purple Montain Observatory (PMO), província de Nanjing, China. É uma instalação da Chinese Academy of Sciences localizada a 267 metros de altitude. O PMO 13.7-m tem como um de seus focos de pesquisa a formação estelar através do universo.

Foi observada a transição $J=1-0$ das espécies ${ }^{12} \mathrm{CO}(115.27 \mathrm{GHz}),{ }^{13} \mathrm{CO}(110.20 \mathrm{GHz}) \mathrm{e}$ $\mathrm{C}^{18} \mathrm{O}(109.78 \mathrm{GHz})$. As observações foram realizadas em Junho de 2012. O modo de observação On-The-Fly (OTF) foi aplicado para mapear uma região de $21^{\prime} \times 25^{\prime}$ centrada em $\alpha_{2000}=$ $18^{h} 14^{m} 01^{s} .361$ e $\delta_{2000}=-17^{\circ} 28^{\prime} 23^{\prime \prime} .14$.

Dos métodos de observação em prato único, o mapeamento OTF é uma técnica na qual o telescópio é conduzido de forma suave e rápida através de um campo, enquanto os dados e as informações de posição da antena são registrados continuamente. A precisão do resultado é melhor porque, uma vez que o campo é coberto rapidamente, as propriedades da atmosfera e do sistema (incluindo o apontamento da antena e a calibração) mudam menos em comparação com outras técnicas convencionais. Além disso, as condições meteorológicas são uniformes sobre a região mapeada. Para produzir a imagem final, os espectros individuais são colocados em uma grade e, em seguida, é realizada uma média.

A largura do feixe à meia potência (HPBW, do inglês Half Power Beam Width) pode ser bem ajustada por:

$$
H P B W=k \frac{\lambda}{D}, \quad \text { em radianos }
$$

onde $k$ é um fator que varia sutilmente conforme o telescópio, $\lambda$ é o comprimento de onda corres- 
pondente e $D$ é o diâmetro do telescópio, sendo que $\lambda$ e $D$ precisam estar na mesma unidade. Para as observações realizadas na antena PMO 13.7-m adotamos $k=1.166 \mathrm{e}$, portanto, o tamanho do beam é HPBW $=\sim 52$ arcsec.

Usamos um arranjo de 9 beams de receptores SIS 11 no front end (Shan et al., 2012). A eficiência do feixe principal (main beam efficiency) no centro do arranjo $3 \times 3$ é de aproximadamente 0.44 a $115 \mathrm{GHz}$, e 0.48 a 110 GHz. Espectrômetros de Transformada Rápida de Fourier (FFTS) foram usados para digitalizar os sinais e alocar $1 \mathrm{GHz}$ em 16.384 canais. Portanto, nossa resolução espectral é de cerca de $61 \mathrm{kHz}$, o que corresponde a resoluções em velocidade de 0.16 $\mathrm{km} \mathrm{s}^{-1}$ a $115 \mathrm{GHz}$, e $0.17 \mathrm{~km} \mathrm{~s}^{-1}$ a $110 \mathrm{GHz}$ e $109 \mathrm{GHz}$.

A condição de tempo nublado durante as observações levaram as temperaturas de sistema a atingirem $550 \mathrm{~K}$ e $350 \mathrm{~K}$ a $115 \mathrm{GHz}$ e $110 \mathrm{GHz}$, respectivamente. Isso resultou em ruídos (rms) de $1.7 \mathrm{~K}$ e $1.2 \mathrm{~K}$ na temperatura de brilho para ${ }^{12} \mathrm{CO} J=1-0$ e ${ }^{13} \mathrm{CO} J=1-0$. Tal ruído pode ter tornado sinais relativamente fracos em sinais indetectáveis.

No entanto, as regiões com forte emissão em linha podem ser validamente investigadas. A informação de velocidade fornecida por estes dados revela, de forma convincente, a cinemática da bolha e as condições moleculares em alguns sub-regiões.

\subsection{Observações com o IRAM 30-m}

IRAM 30-m é um telescópio do Instituto de Radioastronomia Milimétrica (Institut de Radioastronomie Milimétrique ${ }^{2}$ ) instalado no Pico Veleta, na Espanha. Com um diâmetro de 30 metros, é um dos maiores e mais sensíveis rádio telescópios que detecta ondas milimétricas nos dias de hoje. Trata-se de uma single dish (antena de prato único) bastante adequada para detectar fontes distantes ou com baixa intensidade. A espectroscopia em alta resolução nos permite estudar a interação, a composição química e a formação de estrelas dentro de nuvens moleculares na nossa Galáxia.

As observações com o telescópio IRAM 30-m foram realizadas em Setembro de 2015. Utilizamos duas das quatro bandas do EMIR (Eight MIxer Receiver): a banda E090, centrada aproximadamente em $90 \mathrm{GHz}$, e a banda E150, centrada aproximadamente em $150 \mathrm{GHz}$, para mapear a emissão de possíveis traçadores de formação estelar na região de N10. A Figura 3.1 mostra a

\footnotetext{
${ }^{1}$ Superconductor-Insulator-Superconductor

${ }^{2}$ http://www.iram-institute.org/
} 
transmissão atmosférica para as bandas do receptor EMIR ${ }^{3}$

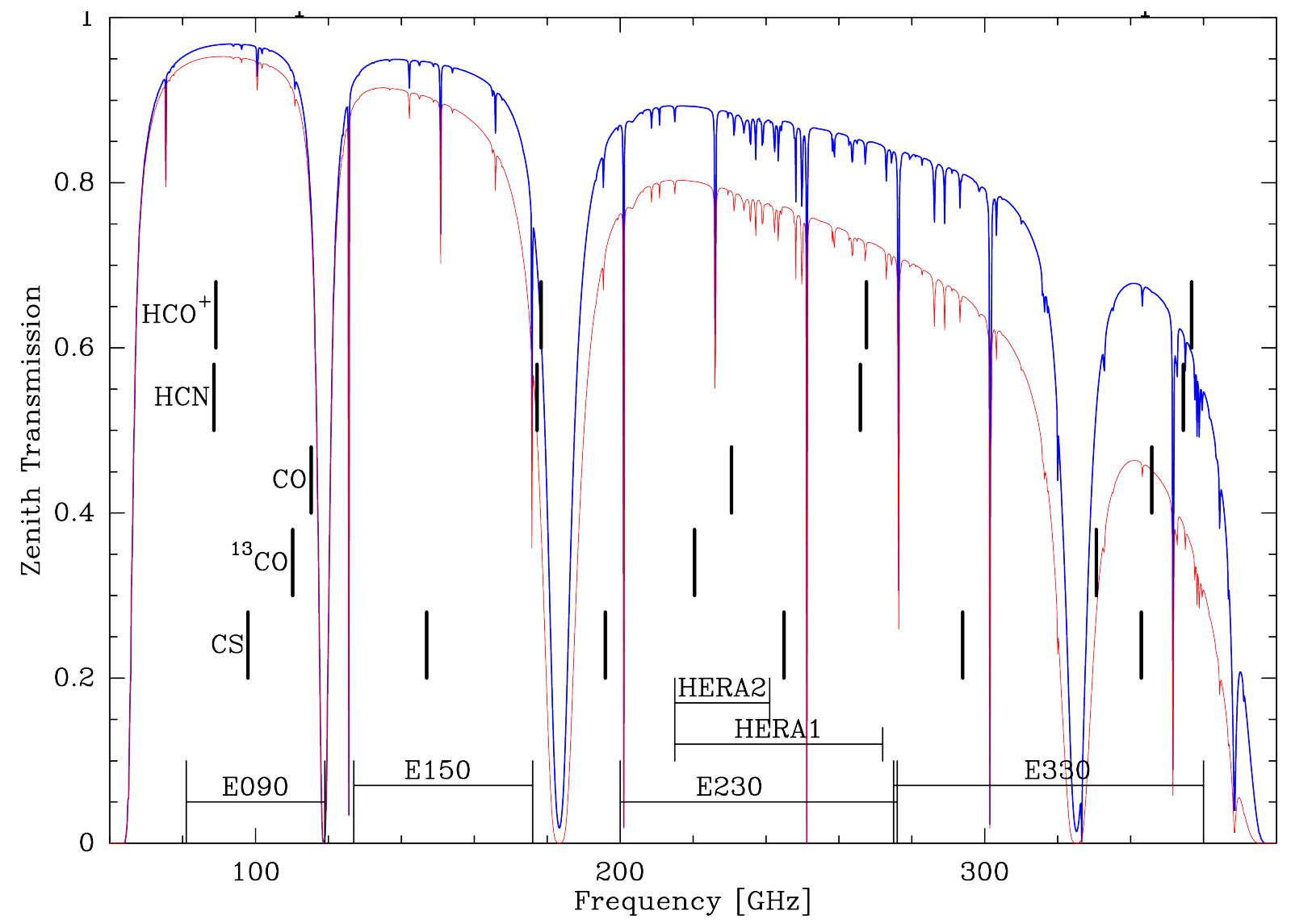

Figura 3.1: Transmissão atmosférica entre 60 e $400 \mathrm{GHz}$ para dois valores de vapor d'água precipitável (pwv, do inglês precipitable water vapor): $2 \mathrm{~mm}$ em azul e $4 \mathrm{~mm}$ em vermelho. As bandas EMIR são mostradas abaixo, com as frequências de algumas transições moleculares importantes.

Nós cobrimos um campo de $25 \operatorname{arcmin}^{2}$ no modo On-The-Fly, campo esse grande o suficiente para envolver a nebulosa ionizada e a nuvem molecular ao redor. Observamos com o receptor EMIR nas bandas E090 (83-117 GHz) e E150 (129-174 GHz), usando o modo de observação OTF/PSW (Position Switching). A calibração foi verificada com relação à Saturno e as condições meteorológicas estavam razoáveis e bastante estáveis durante as sessões de observação.

A eficiência do feixe principal $\left(B_{e f f}\right)$ e a largura do feixe à meia potência $(H P B W)$ variam em função da frequência observada. Para frequências observadas com o E090, a eficiência do feixe principal é $B_{\text {eff }}=0.81$, e com o E150 esse valor é $B_{e f f}=0.93$. A Tabela 3.1 apresenta os valores de $H P B W$ para as linhas de emissão das moléculas mapeadas no modo OTF.

\footnotetext{
${ }^{3}$ Referência: http://www.iram.es/IRAMES/mainWiki/EmirforAstronomers\#EMIR_bands
} 
Tabela 3.1 - Tamanho do feixe à meia potência (HPBW) para cada linha observada no telescópio IRAM 30-m.

\begin{tabular}{l|c|c}
\hline \hline Linha & $\begin{array}{c}\text { Frequência } \\
(\mathrm{GHz})\end{array}$ & $\begin{array}{c}\text { HPBW } \\
(\text { arscec })\end{array}$ \\
\hline $\mathrm{HCO}^{+}(1-0)$ & 89.188 & 27.58 \\
$\mathrm{HCN}(1-0)$ & 88.613 & 27.76 \\
$\mathrm{SiO}(2-1)$ & 86.847 & 28.33 \\
$\mathrm{~N}_{2} \mathrm{H}^{+}(1-0)$ & 93.173 & 26.40 \\
$\mathrm{CS}(3-2)$ & 146.969 & 13.40 \\
\hline
\end{tabular}

Por último, a fim de obter uma compreensão mais aprofundada sobre as propriedades químicas do gás molecular em núcleos pré/protoestelares, realizamos integração profunda na direção da condensação mais brilhante de N10. Integração profunda por 15 minutos, utilizando a banda E090 do receptor EMIR, nos permitiu cobrir um intervalo espectral muito interessante ao longo da banda em $3 \mathrm{~mm}$. 


\section{Capítulo 4}

\section{Resultados}

\subsection{Observações com o PMO 13.7-m}

As transições $J=1-0$ dos isótopos ${ }^{12} \mathrm{CO},{ }^{13} \mathrm{CO}$ e $\mathrm{C}^{18} \mathrm{O}$ foram observadas simultaneamente. Forte emissão de ${ }^{12} \mathrm{CO}$ e ${ }^{13} \mathrm{CO}$ foram observadas, mas a emissão de $\mathrm{C}^{18} \mathrm{O}$ é fraca e não a analisamos nesse trabalho. A Figura 4.1 apresenta as linhas espectrais observadas. Os painéis da esquerda são os espectros médios da região de $21^{\prime} \times 25^{\prime}$ centrada em $\alpha_{2000}=18^{h} 14^{m} 01^{s} .361 \mathrm{e}$ $\delta_{2000}=-17^{\circ} 28^{\prime} 23^{\prime \prime} .14\left(1=13.188^{\circ}, \mathrm{b}=0.039^{\circ}\right)$.
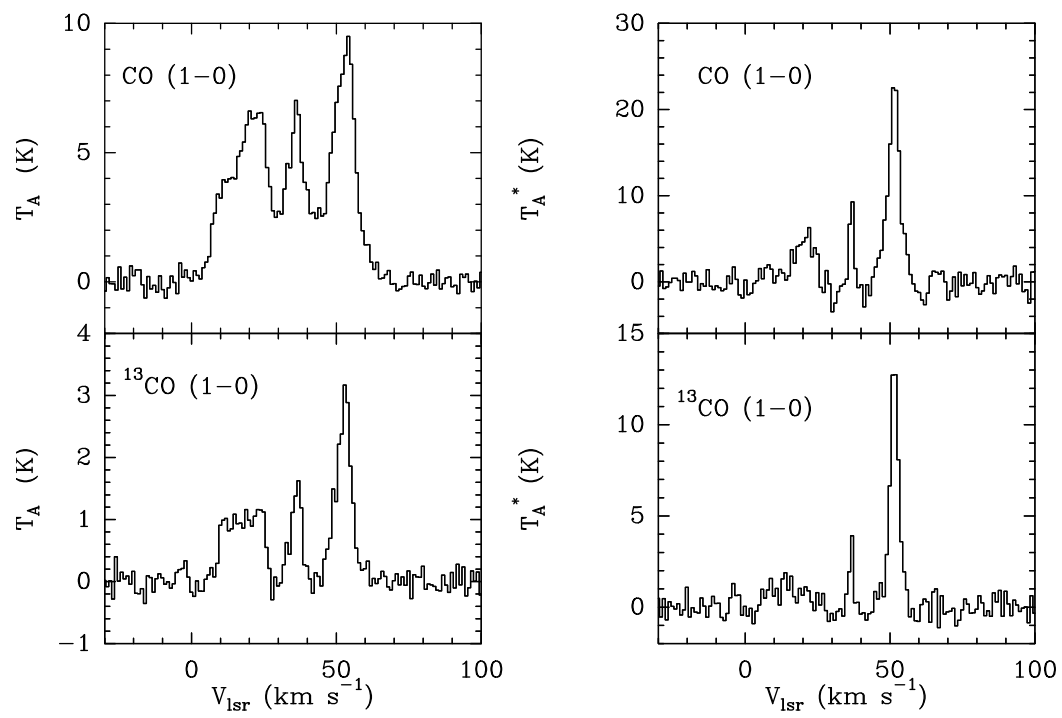

Figura 4.1: Linhas espectrais. A coluna à esquerda mostra o espectro médio da região observada, para ${ }^{12} \mathrm{CO}(1-0)$ no painel acima e para ${ }^{13} \mathrm{CO}(1-0)$ no painel abaixo. A coluna à direita apresenta o espectro dessas duas linhas na posição do pico de emissão, na posição $\alpha_{2000}=18^{h} 14^{m} 10^{s} .03$ e $\delta_{2000}=-17^{\circ} 27^{\prime} 35^{\prime \prime} .3\left(l=13.21^{\circ}, b=0.037^{\circ}\right)$. 
A emissão de ${ }^{12} \mathrm{CO}$ e ${ }^{13} \mathrm{CO}$ que detectamos nos permite identificar três picos de velocidade: a 20, a 37 e a $52 \mathrm{~km} \mathrm{~s}^{-1}$, aproximadamente. As velocidades centrais e a largura das linhas observadas foram determinados por meio do ajuste Gaussiano utilizando o software CLASS, do pacote GILDAS 1 Nesta tese vamos nos referir às velocidades sempre em termos do padrão local de repouso (LSR, do inglês Local Standard of Rest).

A Figura 4.2 dispõe o mapa de canais da emissão ${ }^{12} \mathrm{CO}$ e a Figura 4.3 mostra o mapa de canais da emissão ${ }^{13} \mathrm{CO}$. A imagem de fundo em todos os canais é a emissão contínua a $8.0 \mu \mathrm{m}$. Ajustamos os canais incrementando a velocidade de 1 em $1 \mathrm{~km} \mathrm{~s}^{-1}$, em um intervalo de 45 a 62 $\mathrm{km} \mathrm{s}^{-1}$. Existe uma forte correlação entre a emissão de ${ }^{12} \mathrm{CO}$ e ${ }^{13} \mathrm{CO}$, especialmente no intervalo $49-53 \mathrm{~km} \mathrm{~s}^{-1}$.

\footnotetext{
${ }^{1}$ http://www.iram.fr/IRAMFR/GILDAS
} 


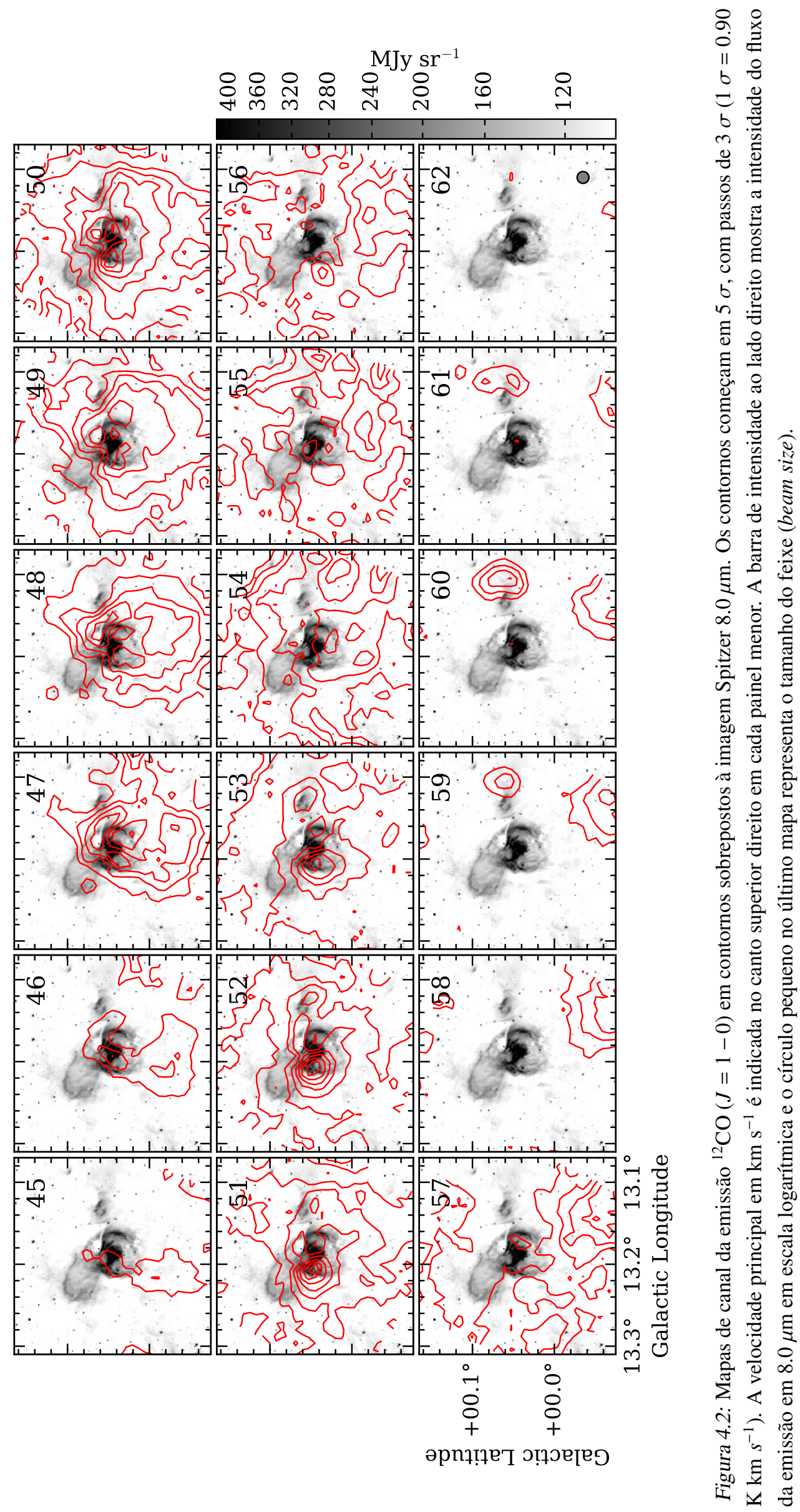




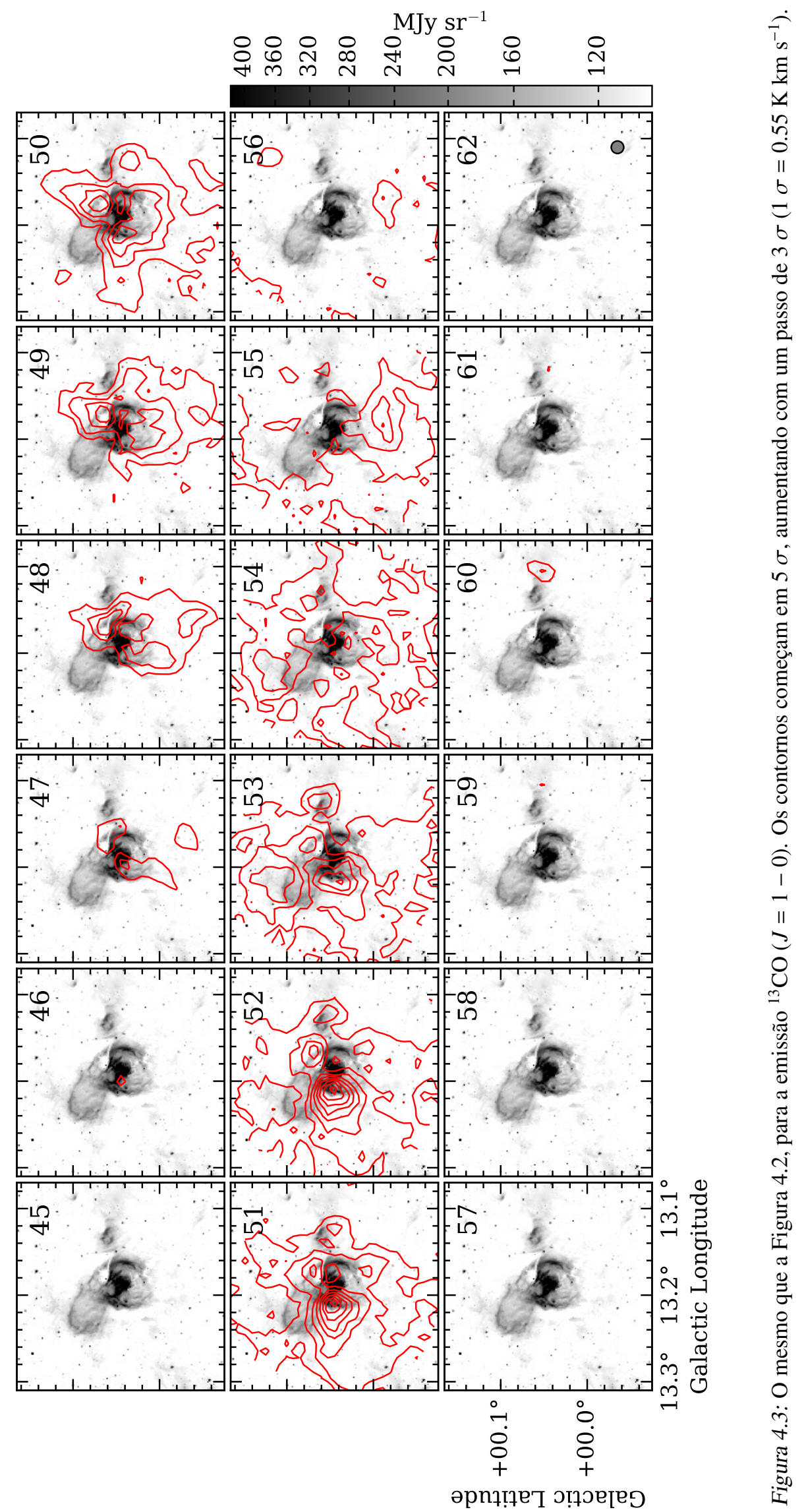


Devemos ser cuidadosos ao associar uma condensação de poeira com N10, pois uma condensação pode estar sendo observada na direção da bolha e não estar fisicamente associada a ela, apenas posicionada ao longo da mesma linha de visada. Esta questão pode ser resolvida com a informação da velocidade.

$\mathrm{Na}$ Figura 4.1 podemos perceber uma componente larga centrada em $\sim 20 \mathrm{~km} \mathrm{~s}^{-1}$, que tem a menor intensidade entre os três picos. A componente centrada em $\sim 37 \mathrm{~km} \mathrm{~s}^{-1}$ apresenta um perfil mais estreito que a componente anterior, e intensidade menor se comparada com a componente centrada em $\sim 52 \mathrm{~km} \mathrm{~s}^{-1}$. As velocidades encontradas na literatura para diferentes linhas de emissão associadas a N10 variam de 48.5 a $54.1 \mathrm{~km} \mathrm{~s}^{-1}$, como mostra a Tabela 4.1 . Isso nos leva a adotar a componente com pico em $52 \mathrm{~km} \mathrm{~s}^{-1}$ como a componente fisicamente relacionada a nossa fonte.

Tabela 4.1 - Velocidades obtidas na direção da bolha N10, através de diferentes métodos.

\begin{tabular}{ccc}
\hline \hline $\begin{array}{c}\text { Velocidade } \\
\left(\mathrm{km} \mathrm{s}^{-1}\right)\end{array}$ & Método & Referência \\
\hline 54.1 & Região HII, linha de recombinação em rádio & Lockman (1989) \\
48.5 & Emissão a 6.7 GHz de maser de metanol & Szymczak et al. (2000) \\
54.1 & Linha de absorção HI & Pandian et al. (2008) \\
50.2 & Linha de emissão de CO & Beaumont \& Williams (2010) \\
48.5 & Linha de inversão de NH & Wienen et al. (2012) \\
54.1 & Dados WISE da região HII & Anderson et al. (2014) \\
\hline
\end{tabular}

As velocidades em torno do pico de emissão em $52 \mathrm{~km} \mathrm{~s}^{-1}$ em nossas observações variam de 48 a $53 \mathrm{~km} \mathrm{~s}^{-1}$. Com o intuito de verificar a correspondência entre a distribuição física do conteúdo molecular e a bolha vista em infravermelho, sobrepusemos contornos da emissão de ${ }^{12} \mathrm{CO}$ e ${ }^{13} \mathrm{CO}$ da componente estreita sobre a imagem Spitzer $8.0 \mu \mathrm{m}$ na Figura 4.4 . A distribuição espacial de ${ }^{12} \mathrm{CO}$ mostra duas estruturas principais que parecem estar relacionadas com a emissão a $8.0 \mu \mathrm{m}$ emission, e ${ }^{13} \mathrm{CO}$ apresenta dois clumps na borda da morfologia anelar de $\mathrm{N} 10$, nas mesmas posições que as estruturas em ${ }^{12} \mathrm{CO}$. 

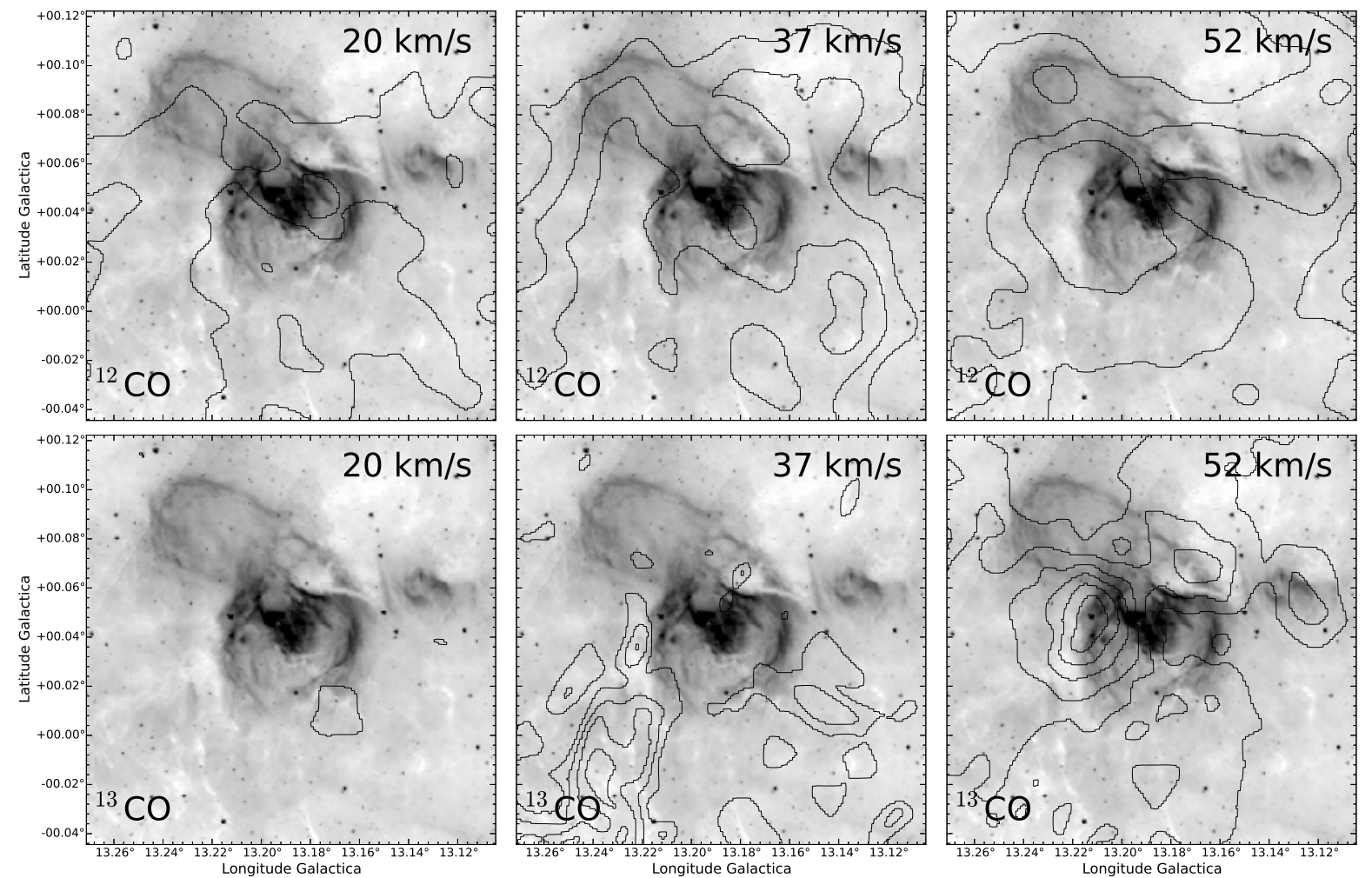

Figura 4.4: Os painéis acima mostram os contornos da emissão ${ }^{12} \mathrm{CO}$ centrada em $20 \mathrm{~km} \mathrm{~s}^{-1}$ (à esquerda), $37 \mathrm{~km}$ $\mathrm{s}^{-1}$ (no centro) e $52 \mathrm{~km} \mathrm{~s}^{-1}$ (à direita), de 4.5 a $12.6 \mathrm{~K}$ com passos de $2.7 \mathrm{~K}$. Os painéis abaixo apresentam contornos da distribuição da emissão ${ }^{13} \mathrm{CO}$ em $20 \mathrm{~km} \mathrm{~s}^{-1}$ (à esquerda), $37 \mathrm{~km} \mathrm{~s}^{-1}$ (no centro) e $52 \mathrm{~km} \mathrm{~s}^{-1}$ (à direita), de 2.75 a $11 \mathrm{~K}$ com passos de $1.65 \mathrm{~K}$. Todos os painéis exibem a imagem Spitzer $8.0 \mu \mathrm{m}$ como plano de fundo. 


\section{- Parâmetros observados}

$\mathrm{Na}$ discussão a seguir adotaremos a identificação para os clumps de acordo com a Figura 4.5 para nos referirmos às condensações identificadas. Devido à fraca resolução angular do PMO 13.7-m (nas nossas observações, o tamanho do feixe equivale a 0.9 pc), o tamanho dessas condensações podem ser menor do que 0.9 pc. Classificamos como clumps aquelas condensações nas quais a formação de aglomerados estelares podem ocorrer (Bergin \& Tafalla, 2007).

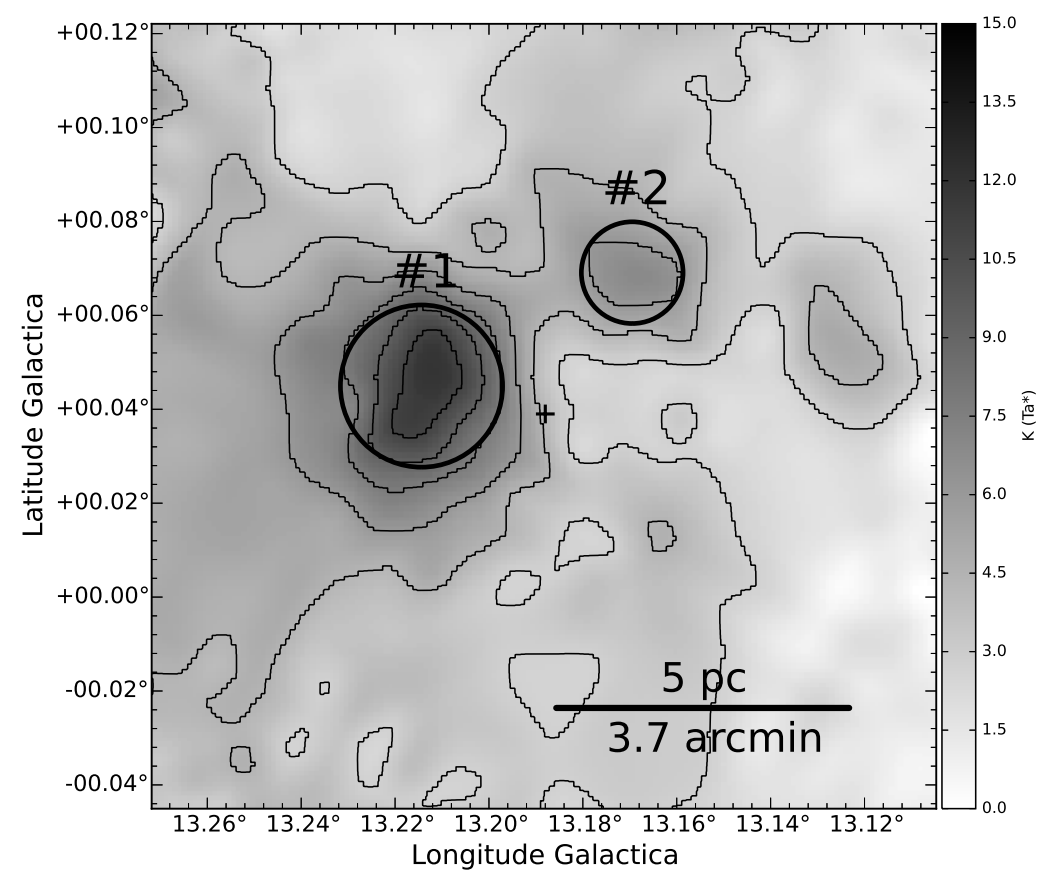

Figura 4.5: Região dos clumps identificados no mapa de intensidade de ${ }^{13} \mathrm{CO}$ centrado em $52 \mathrm{~km} \mathrm{~s}^{-1}$, em escala de cinza. O Clump \#1 e o Clump \#2 parecem estar fisicamente associados à bolha N10. Contornos em preto mostram também a emissão em ${ }^{13} \mathrm{CO}$ de 2.75 a $11 \mathrm{~K}$, em passos de $1.65 \mathrm{~K}$. A cruz preta indica o centro da região HII na bolha N10.

Utilizamos o CLASS/GILDAS para estimar parâmetros por meio de ajustes Gaussianos e obtivemos a velocidade central $\left(\mathrm{V}_{L S R}\right)$, a temperatura de antena $\left(\mathrm{T}_{A}^{*}\right)$ e a largura à meia altura $\left(\triangle \mathrm{V}_{F W H M}\right.$, FWHM do inglês Full Width at Half Maximum) das linhas observadas. Os valores são mostrados na Tabela 4.2.

\section{- Parâmetros físicos calculados}

Para melhor compreender o status evolutivo dos clumps, calculamos as propriedades dos dois clumps identificados em nossas observações de CO. A massa dos clumps foi estimada com o auxílio do software Miriad (Sault et al., 1995). 
A massa do gás molecular em uma condensação é calculada através da intensidade da linha de emissão sob a hipótese de equilíbrio termodinâmico local (LTE, do inglês Local Thermodynamic Equilibrium). Com a equação de transferência radiativa de Garden et al. (1991), calculamos a massa dos clumps sob a condição de LTE, $M_{L T E}$, seguindo a relação:

$$
M_{L T E}=\frac{4}{3} \pi R^{3} m_{H_{2}} \mu_{g} n_{H_{2}}
$$

onde $M_{L T E}$ é dada em massas solares $M_{\odot}, m_{H_{2}}$ é a massa da molécula de hidrogênio, $\mu_{g}=1.36$ é o peso atômico médio do gás e $n_{H_{2}}$ é a densidade em número de moléculas por unidade de volume. $\mathrm{R}$ é o raio médio da condensação é obtido pela relação $R=\sqrt{b_{m a j} \times b_{\min }} / 2$, sendo $b_{\text {maj }}$ e $b_{\text {min }}$ os semi-eixos maior e menor da elipse, respectivamente, obtidos com a utilização do software Miriad.

Considerando $n_{\mathrm{H}_{2}}=N_{\mathrm{H}_{2}} / 2 R$, devemos primeiramente estimar o valor da densidade colunar $N_{H_{2}}$ por meio da densidade colunar de CO. De acordo com Garden et al. (1991), a densidade colunar $N$ de uma molécula linear rígida, sob a condição de LTE, pode ser expressa por:

$$
N=\frac{3 k}{8 \pi^{3} B \mu^{2}} \times \frac{\exp \left[h B J(J+1) / k T_{e x}\right]}{(J+1)} \times \frac{\left(T_{e x}+h B / 3 k\right)}{\left[1-\exp \left(-h v / k T_{e x}\right]\right.} \times \int \tau \mathrm{d} v
$$

onde B é a constante rotacional, J é o número quântico rotacional do estado inferior da transição observada e $\mu$ é o dipólo elétrico da molécula. $T_{e x}$ é a temperatura de excitação e $\tau$ é a profundidade óptica de 48 a $53 \mathrm{~km} \mathrm{~s}^{-1}$.

A temperatura de brilho $\left(T_{r}\right)$ pode ser obtida diretamente da observação, uma vez que esta é a medida da intensidade da fonte. Sendo a temperatura de excitação $\left(T_{e x}\right)$ medida em função de $T_{r}$, estimamos (Garden et al., 1991):

$$
T_{r}=\frac{h v}{k} \times\left[\frac{1}{\exp \left(h v / k T_{e x}\right)-1}-\frac{1}{\exp \left(h v / k T_{b g}\right)-1}\right] \times[1-\exp (-\tau)] f
$$

Tabela 4.2 - Parâmetros das linhas dos espectros médios da região observada, ilustrados na coluna esquerda na Figura 4.1

\begin{tabular}{cccc}
\hline \hline Linha & $\begin{array}{c}\mathrm{V}_{L S R} \\
\left(\mathrm{~km} \mathrm{~s}^{-1}\right)\end{array}$ & $\begin{array}{c}\mathrm{T}_{A}^{*} \\
(\mathrm{~K})\end{array}$ & $\begin{array}{c}\Delta \mathrm{V}_{F W H M} \\
\left(\mathrm{~km} \mathrm{~s}^{-1}\right)\end{array}$ \\
\hline${ }^{12} \mathrm{CO}$ & 52.6 & 8.3 & 9.2 \\
${ }^{13} \mathrm{CO}$ & 52.6 & 2.9 & 6.1 \\
\hline
\end{tabular}


onde $T_{r}, T_{e x}$ e $T_{b g}$ são expressas em K e $T_{b g}=2.73 \mathrm{~K}$ é a temperatura da radiação cósmica de fundo. Para as observações no PMO 13.7-m nesses comprimeitos de onda, assumimos um "fator de preenchimento" (ou seja, a fração do feixe preenchida pela emissão, filling factor) $f=1$.

Assumindo que $T_{e x}$ é a mesma para ${ }^{12} \mathrm{CO}$ e para ${ }^{13} \mathrm{CO}$, as profundidades óptica de ambas as linhas podem ser obtidas comparando diretamente a medida das suas temperaturas de brilho $T_{r}$ (Garden et al., 1991):

$$
\frac{T_{r}\left({ }^{12} C O\right)}{T_{r}\left({ }^{13} C O\right)} \approx \frac{1-\exp \left(-\tau_{12}\right)}{1-\exp \left(-\tau_{13}\right)}
$$

Adotamos uma razão de isótopo de $\left[{ }^{12} \mathrm{CO}\right] /\left[{ }^{13} \mathrm{CO}\right]=60$ (Deharveng et al., 2008), o que implica $\tau_{12} / \tau_{13}=60$. Assim, podemos obter a temperatura de excitação $T_{e x}$ aplicando a relação 4.4 na equação 4.3 . Utilizando o valor de $T_{e x}$ estimado para a linha ${ }^{13} \mathrm{CO}$, adotando a razão de isótopo de $\left[{ }^{12} \mathrm{CO}\right] /\left[{ }^{13} \mathrm{CO}\right]=60$ e assumindo um valor canônico de $10^{-4}$ para a razão de abundância $[\mathrm{CO}] /\left[\mathrm{H}_{2}\right]$, por fim obtemos obtemos a densidade colunar para o hidrogênio utilizando a equação 4.2 . Dessa forma, a linha ${ }^{13} \mathrm{CO}$ traça a densidade colunar dos clumps \#1 e \#2, conforme listamos na Tabela 4.3 ,

Com a dispersão de velocidade obtida e a massa sob LTE, podemos estimar a condição virial comparando a massa do gás com a massa virial $M_{\text {viral }}$. Em uma nuvem na qual a média temporal da energia cinética é igual à metade da média temporal da energia potencial, o sistema estará em equilíbrio virial. A hipótese de que um sistema gravitacionalmente ligado se encontra em equilíbrio virial é bastante usada em Astrofísica para estimar sua massa (Huang, 1954). A massa virial $M_{\text {viral }}$ é dada por Ungerechts et al. (2000) através da expressão:

$$
\frac{M_{\text {virial }}}{M_{\odot}}=2.10 \times 10^{2}\left(\frac{R}{p c}\right)\left(\frac{\Delta V_{F W H M}}{k m s^{-1}}\right)^{2}
$$

onde $R$ é o raio médio do clump.

A massa de Jeans $M_{J}$ é a massa acima da qual uma nuvem de gás irá colapsar, para uma dada densidade e temperatura, quando a atração gravitacional supera a pressão do gás. Pode ser calculada de acordo com Stahler \& Palla (2005):

$$
\frac{M_{\text {Jeans }}}{M_{\odot}}=\left(\frac{T}{10 K}\right)^{3 / 2}\left(\frac{n_{H_{2}}}{10^{4} c m^{-3}}\right)^{-1 / 2}
$$


Os resultados são apresentados na Tabela 4.3, onde as colunas de 2 a 7 listam, respectivamente, os seguintes parâmetros dos clumps identificados em ${ }^{13} \mathrm{CO}$ : raio médio $(\mathrm{R})$, temperatura de excitação $\left(T_{e x}\right)$, densidade colunar de hidrogênio $\left(N_{H_{2}}\right)$, densidade $\left(n_{H_{2}}\right)$, massa calculada sob a hipótese de LTE $\left(M_{L T E}\right)$, massa virial $\left(M_{\text {virial }}\right)$ e massa de Jeans $\left(M_{\text {Jeans }}\right)$.

Tabela 4.3 - Parâmetros calculados para os clumps observados em ${ }^{13} \mathrm{CO}$.

\begin{tabular}{cccccccc}
\hline \hline clump & $\begin{array}{c}\mathrm{R} \\
(\mathrm{pc})\end{array}$ & $\begin{array}{c}\mathrm{T}_{e x} \\
(\mathrm{~K})\end{array}$ & $\begin{array}{c}\mathrm{N}_{H_{2}} \\
\left(10^{22} \mathrm{~cm}^{-2}\right)\end{array}$ & $\begin{array}{c}\mathrm{n}_{H_{2}} \\
\left(10^{3} \mathrm{~cm}^{-3}\right)\end{array}$ & $\begin{array}{c}\mathrm{M}_{L T E} \\
\left(10^{3} \mathrm{M}_{\odot}\right)\end{array}$ & $\begin{array}{c}\mathrm{M}_{\text {virial }} \\
\left(10^{3} \mathrm{M}_{\odot}\right)\end{array}$ & $\begin{array}{c}\mathrm{M}_{\text {Jeans }} \\
\left(10^{3} \mathrm{M}_{\odot}\right)\end{array}$ \\
\hline$\# 1$ & 1.1 & 16.8 & 4.1 & 4.2 & 2.6 & 9.3 & 7.0 \\
$\# 2$ & 1.2 & 12.9 & 3.3 & 3.9 & 1.5 & 7.8 & 4.7 \\
\hline
\end{tabular}




\subsection{Distribuição de poeira fria}

A emissão térmica da poeira fria é a principal responsável pela distribuição do contínuo em $870 \mu \mathrm{m}$ na direção de $\mathrm{N} 10$, enquanto a emissão em $8.0 \mu \mathrm{m}$ tem origem nos hidrocarbonetos policíclicos aromáticos (PAHs) excitados pelos fótons UV. A Figura 4.6 mostra a emissão em $870 \mu \mathrm{m}$ em escala de cinza e contornos (à esquerda), e os mesmos contornos da emissão de poeira fria são sobrepostos na imagem Spitzer $8.0 \mu$ m (à direita).

Os dois clumps $870 \mu \mathrm{m}$ coicidem com as condensações moleculares detectadas no pico de velocidade de $52 \mathrm{~km} \mathrm{~s}^{-1}$ mostradas nos painéis a direita da Figura 4.4. Nesta seção, calculamos os parâmetros físicos para a condensação mais densa, em destaque na Figura 4.7 .

Se considerarmos a emissão em $870 \mu \mathrm{m}$ como um resultado dos grãos de poeira térmicos, podemos calcular a massa total $\left(M_{t o t}\right)$ dessa condensação seguindo a relação citada por Deharveng et al. (2010):

$$
M_{t o t}=100 \frac{\mathrm{S}_{870 \mu m} D^{2}}{\kappa_{870 \mu m} B_{870 \mu m}\left(\mathrm{~T}_{\text {dust }}\right)},
$$

onde $M_{t o t}$ é dada em gramas, $\mathrm{S}_{870 \mu m}$ é a densidade de fluxo total da emissão $870 \mu \mathrm{m}$ em Jy, $D$ em kpc, $\kappa_{870 \mu m}$ é a opacidade da poeira por unidade de massa $\mathrm{em} \mathrm{cm}^{2} \mathrm{~g}^{-1}$ e $B_{870 \mu m}$ é a função de Planck (para uma dada temperatura de poeira $\mathrm{T}_{d u s t}$ ) em Jy. Aqui assumimos que $T_{d u s t}=20 \mathrm{~K}$, $\kappa_{870 \mu m}=1.8 \mathrm{~cm}^{2} \mathrm{~g}^{-1}$ e a razão gás-poeira em massa é $\sim 100: 1$ (Kerr \& Knapp, 1974).

Calculamos a densidade colunar de $\mathrm{H}_{2}, \mathrm{~N}\left(\mathrm{H}_{2}\right)$, usando a relação fornecida por Deharveng et al. (2010):

$$
\mathrm{N}\left(H_{2}\right)=\frac{100 F_{870 \mu m}}{\kappa_{870 \mu m} B_{870 \mu m}\left(\mathrm{~T}_{\text {dust }}\right) 2.8 m_{H} \Omega_{\text {beam }}},
$$

onde $N\left(H_{2}\right)$ está em $\mathrm{cm}^{-2}$, o brilho superficial $F_{870 \mu m}$ está expresso em Jy beam ${ }^{-1}$, o ângulo sólido do feixe principal $\Omega_{\text {beam }}$ está em esteradianos e a massa do hidrogênio $\mathrm{m}_{H}$ está em gramas.

Para estimar a dimens]ao física da condensação de poeirra fria, calculamos o raio efetivo decolvoluído de acordo com a relação:

$$
\mathrm{R}_{D}=\left(R_{m a j, D} \times R_{\min , D}\right)^{0.5}=\left(\frac{\theta_{m a j, D}}{2} \times \frac{\theta_{\min , D}}{2}\right)^{0.5}
$$



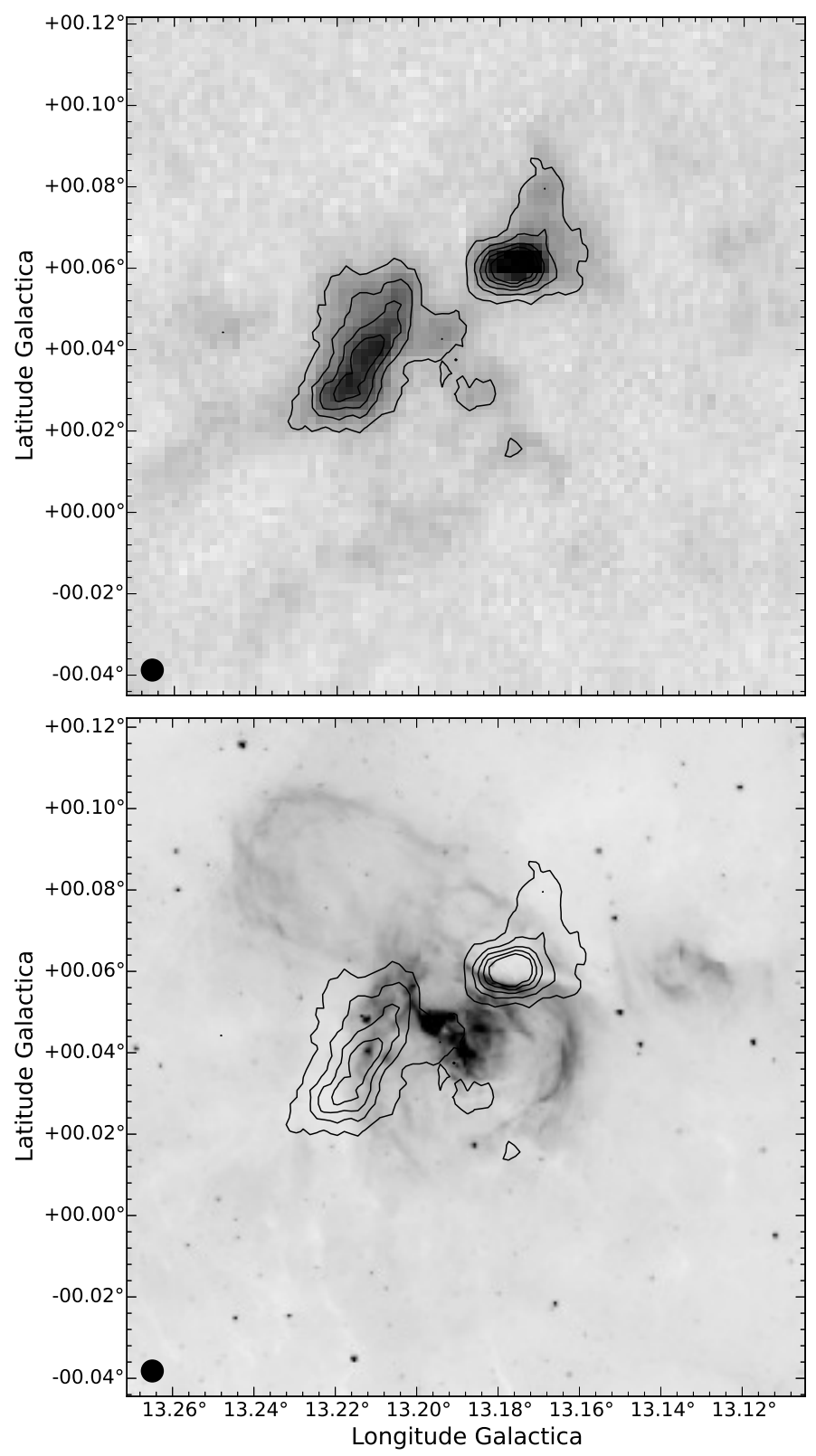

Figura 4.6: Panel superior: emissão $870 \mu \mathrm{m}$ mostrando a distribuição de poeira fria em escala de cinza, de 0.2 a $4.2 \mathrm{Jy}_{\text {beam }}{ }^{-1}$, com contornos de 2 a $5 \sigma$, em passos de $1 \sigma\left(1 \sigma=0.5 \mathrm{Jy} \mathrm{beam}^{-1}\right)$. Painel inferior: imagem Spitzer $8.0 \mu \mathrm{m}$ ao fundo, com os mesmos contornos em $870 \mu \mathrm{m}$ da figura à esquerda. Nas duas imagens, o ponto em preto representa o beam size.

onde a FWHM convoluídas mínima $\left(\theta_{\text {maj,D }}\right)$ e máxima $\left(\theta_{\min , D}\right)$ da condensação foi calculada de acordo:

$$
\theta_{m a j, D}=\sqrt{\theta_{m a j}^{2}-\theta_{H P B W}^{2}} \quad e \quad \theta_{\min , D}=\sqrt{\theta_{m i n}^{2}-\theta_{H P B W}^{2}},
$$

onde $\theta_{\text {maj }}$ e $\theta_{\text {min }}$ são os semi-eixos maior e menor na FWHM, respectivamente, obtidos através 


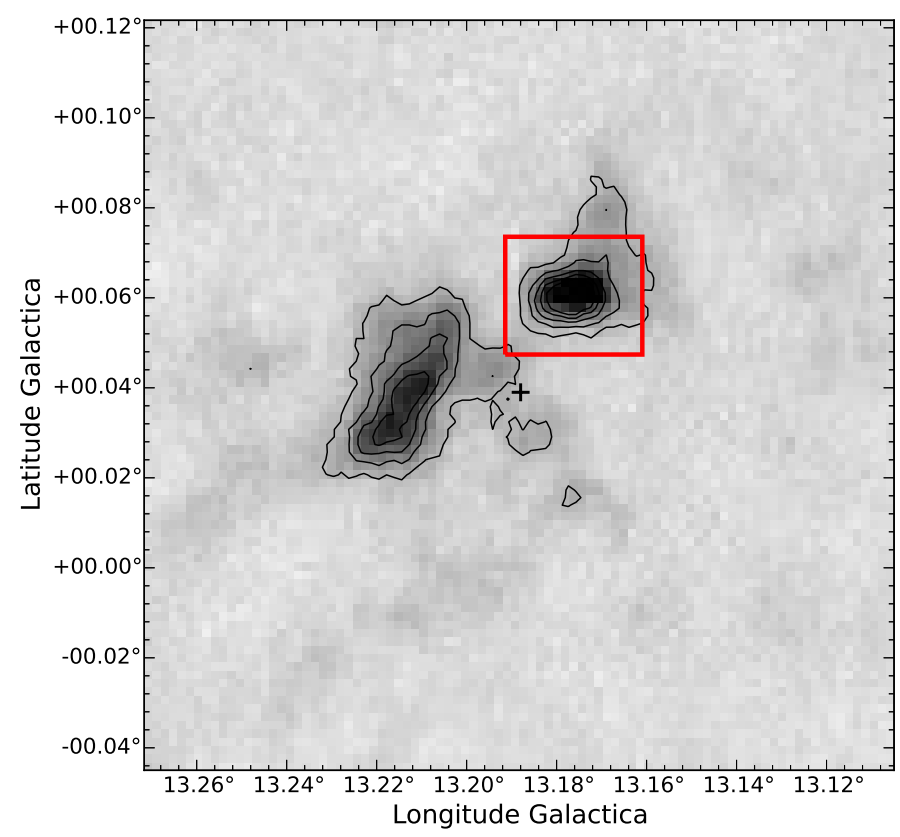

Figura 4.7: Mapa em escala de cinza da emissão $870 \mu \mathrm{m}$, com os mesmos contornos da Figura 4.6 . O retângulo em vermelho destaca a região do clump de poeira fria mais denso, para o qual os parâmetros físicos foram obtidos.

de ajustes realizados com o GreG/GILDAS. O tamanho do feixe à meia altura para $870 \mu m$ é $\theta_{H P B W}=19.2$ arcsec. Encontramos, portanto, que $\theta_{\text {maj }}=44 \operatorname{arcsec}$ e $\theta_{\min }=32 \operatorname{arcsec}$. Adotando uma distância de $4.7 \mathrm{kpc}$ até N10, $R_{m a j, D}=0.90 \mathrm{pc}$ e $R_{m i n, D}=0.58 \mathrm{pc}$, resultando em um raio médio $R_{D}=0.7 \mathrm{pc} \sim 2.2 \times 10^{18} \mathrm{~cm}$.

A densidade média para essa condensação, assumindo uma geometria esférica, foi calculada de acordo com a relação:

$$
n\left(H_{2}\right)=\frac{\mathrm{M}_{t o t}}{4 / 3 \pi R_{D}^{3} \mu_{H} m_{H}},
$$

onde $\mu$ é o peso molecular médio e $m_{H}$ é a massa do hidrogênio atômico. O raio médio deconvoluído é dado em cm. Nós assumimos $\mu_{H}=2.33 \mathrm{~g} \mathrm{e} m_{H}=1.67 \times 10^{-24} \mathrm{~g}$. Estimamos uma densidade média $n\left(H_{2}\right)=1.17 \times 10^{4} \mathrm{~cm}^{-3}$.

\subsection{Gás ionizado}

O gás ionizado associado com N10 pode ser traçado pela emissão em $20 \mathrm{~cm}$. A presença da emissão a $v=1.4 \mathrm{GHz}$ implica que fótons UV criaram uma região HII na parte interna da bolha. Usando o software Greg/GILDAS, nós estimamos um fluxo total em $20 \mathrm{~cm}$ de $F_{20} \mathrm{~cm}=1.17 \mathrm{Jy}$ 
no interior da bolha e calculamos a densidade eletrônica, $n_{e}$, de acordo com Panagia \& Walmsley (1978):

$$
\frac{\mathrm{n}_{e}}{\mathrm{c}^{-3}}=3.113 \times 10^{2}\left(\frac{\mathrm{T}_{e}}{10^{4} K}\right)^{0.25}\left(\frac{\mathrm{S}_{v}}{\mathrm{~J} y}\right)^{0.5}\left(\frac{\mathrm{D}}{\mathrm{k} p c}\right)^{-0.5} \times b(v, \mathrm{~T})^{-0.5} \times \theta_{\mathrm{R}}^{-1.5}
$$

onde $T_{e}$ em K é a temperatura eletrônica, $S_{v}$ é a densidade de fluxo total medida em Jy, $D$ é a distância em kpc e $\theta_{R}$ é o raio angular em arcmin. A função $b(\nu, T)$ é definida como:

$$
b(v, \mathrm{~T})=1+0.3195 \log \left(\frac{\mathrm{T}_{e}}{10^{4} K}\right)-0.2130 \log \left(\frac{v}{1 G H z}\right) .
$$

Assumindo uma temperatura eletrônica $T_{e} \sim 10^{4} \mathrm{~K}$ para a região de emissão livre-livre, a densidade eletrônica é $n_{e} \sim 130 \mathrm{~cm}^{-3}$.

A Figura 4.8 mostra dois picos em contínuo de rádio tanto em escala de cinza quanto em contornos pretos no painel acima. Na mesma figura, o painel abaixo apresenta um desses picos coincidindo com uma estrela do tipo O. O gás ionizado parece estar confinado pela casca da bolha exibida em $8.0 \mu \mathrm{m}$ na cor vermelha.

O número de fótons no contínuo de Lyman que são absorvidos pelo gás na região HII foi estimado por meio do mapa seguindo a relação de Matsakis et al. (1976):

$$
\mathrm{N}_{L y}=7.5 \times 10^{46}\left(\frac{v}{\mathrm{GHz}}\right)^{0.1}\left(\frac{\mathrm{T}_{e}}{10^{4} K}\right)^{-0.45}\left(\frac{\mathrm{S}_{v}}{\mathrm{~J} y}\right)\left(\frac{\mathrm{D}}{\mathrm{k} p c}\right)^{2} .
$$

Estimamos $N_{L y}=1.86 \times 10^{49}$ fótons ionizantes $\mathrm{s}^{-1}$ no contínuo de Lyman, o equivalente a uma única estrela do tipo O, suficiente para manter a frente de ionização (Watson et al., 2008). Considerando um modelo de região HII em expansão no qual o material neutro se acumula entre a frente de ionização e a frente de choque (Deharveng et al., 2010), o gás ionizado está cercado por uma casca de material denso que abriga PAHs, o principal responsável pela emissão em 8.0 $\mu \mathrm{m}$ no infravermelho médio.

Considerando um fluxo no contínuo de Lyman da ordem de $10^{49}$ fótons ionizantes s $^{-1}$, Ma et al. (2013) estimaram uma idade dinâmica $t_{d y n}=9.17 \times 10^{4}$ anos para a bolha. Ainda assim, os autores argumentam que esse valor pode ser maior, uma vez que a densidade do ambiente inicial onde as estrelas teriam se formado originalmente pode ser maior do que a considerada. 

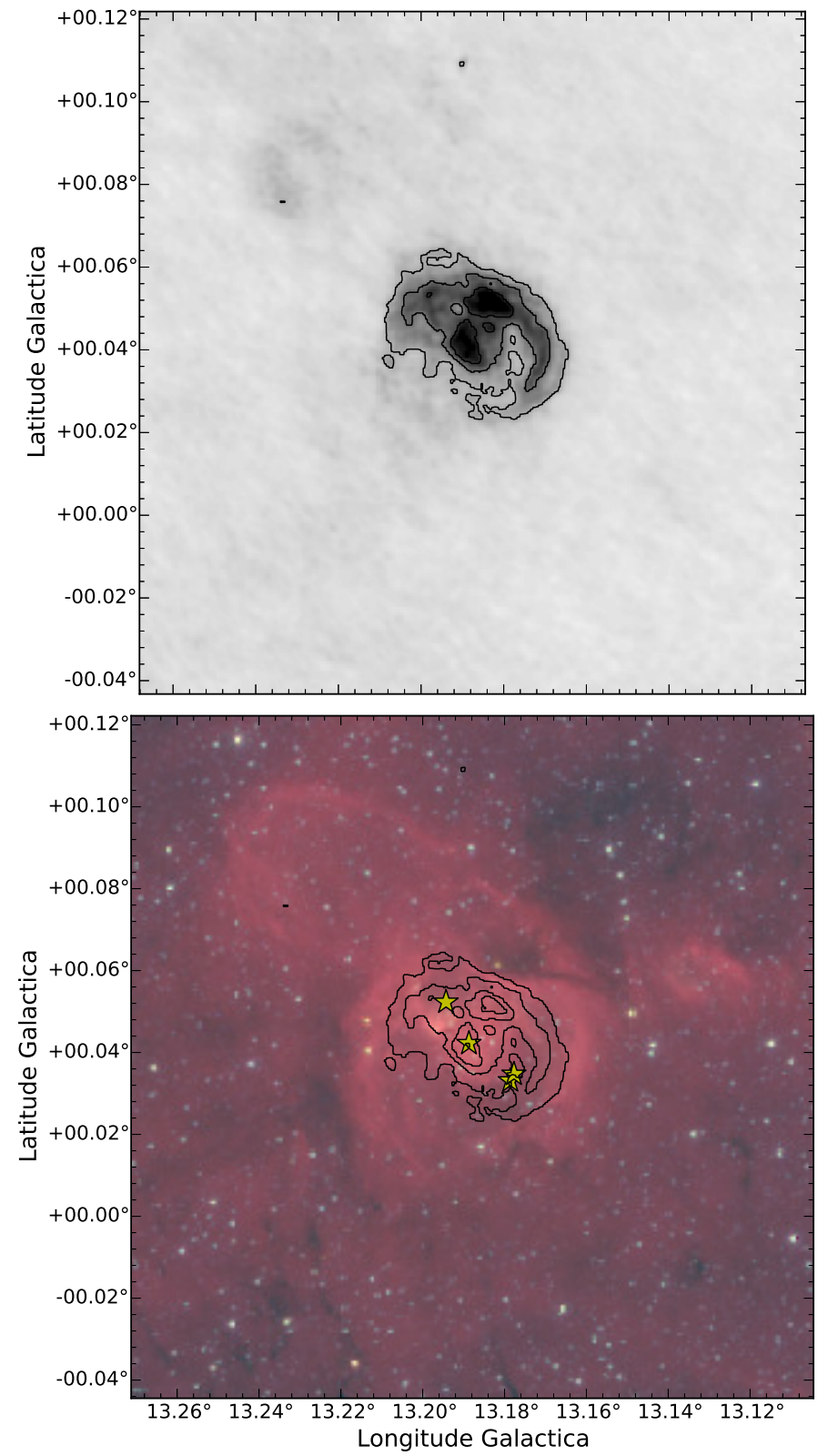

Figura 4.8: Painel superior: intensidade da emissão em $20 \mathrm{~cm}$ em escala de cinza, de 0 a $22 \mathrm{mJy} \mathrm{beam}^{-1}$, com contornos começando em $2 \sigma$ em passos de $2 \sigma\left(1 \sigma=2.8 \mathrm{mJy}_{\text {beam }}{ }^{-1}\right)$. Painel inferior: imagem RGB Spitzer em plano de fundo ( $3.6 \mu \mathrm{m}$ em azul, $4.5 \mu \mathrm{m}$ em verde e $8.0 \mu \mathrm{m}$ em vermelho) com mesmos contornos que o painel superior. Estrelas amarelas marcam a posição das candidatas a estrelas ionizantes listadas na Tabela 2.1. 


\subsection{Presença de poeira morna na região}

Poeira morna também pode ser traçada na bolha N10 por meio da distrubuição da emissão em $24 \mu \mathrm{m}$. A Figura 4.9 mostra a emissão $24 \mu \mathrm{m}$ em escala de cinza. A emissão em $20 \mathrm{~cm}$ é mostrada em contornos.

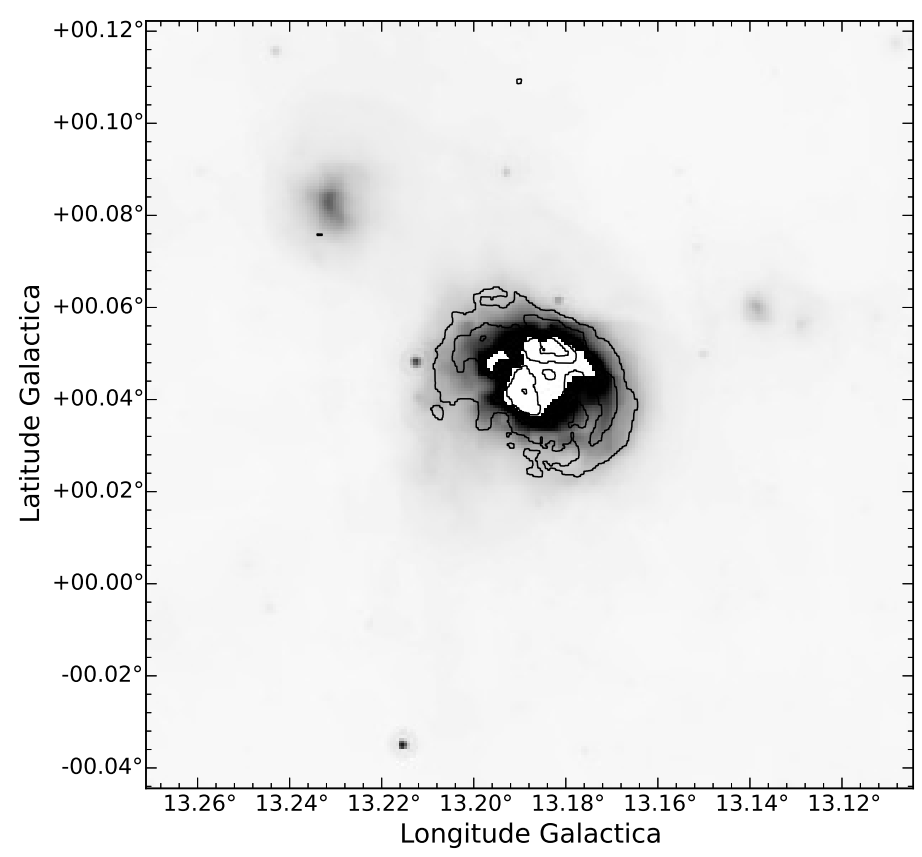

Figura 4.9: Distribuição da emissão em $24 \mu \mathrm{m}$ em escala de cinza de 0 a $1500 \mathrm{MJy} \mathrm{sr}^{-1}$ com os mesmos contornos em $20 \mathrm{~cm}$ da Figura 4.8

Poeira morna e gás ionizado parecem estar bem correlacionados, como o esperado para regiões HII (e.g. Paladini et al., 2012). Podemos perceber que a emissão em $24 \mu \mathrm{m}$ aparece saturada próxima à emissão em $20 \mathrm{~cm}$ devido à sua intensidade.

\section{Distância}

Para esse trabalho, utilizando os modelos de rotação Galáctica circular (e.g. Brand \& Blitz, 1993) é possível estimar as distâncias cinemáticas próxima e distante da fonte. Churchwell et al. (2006) argumentam que bolhas infravermelhas são mais propensas a situarem-se a distâncias cinemáticas próximas, uma vez que objetos do lado mais distante do disco Galáctico podem ser obscurecidos pela extinção interestelar e sofrer contaminação por parte de outras estruturas.

Baseados nas nossas observações de CO e utilizando a velocidade de $52.6 \mathrm{~km} \mathrm{~s}^{-1}$, obtivemos distâncias cinemáticas próxima e distante de $4.7 \mathrm{kpc}$ e $11.3 \mathrm{kpc}$, respectivamente. Este valor é 
compatível com a distância próxima estimada por Szymczak et al. (2000), no valor de 4.4 kpc, usando a emissão do maser de metanol (ver Tabela 4.1).

Uma vez que N10 está localizada no interior do disco da Galáxia, adotamos uma incerteza de $10 \%$ para a distância cinemática (Yuan et al., 2014), resultando na distância de $4.7 \pm 0.5 \mathrm{kpc}$ para N10.

\subsection{Identificação de YSOs no campo de N10}

A distribuição de YSOs desempenha um papel importante na interpretação da dinâmica da região de formação estelar. Para identificar os YSOs presentes na região de N10, adotamos o método descrito por Koenig \& Leisawitz (2014) (daqui em diante KL), baseado nos dados do WISE citep[veja][]wright2010. Em particular, usamos a divulgação AllWISE (Cutri et al. 2011 Cutri et al., 2013).

O catálogo foi acessado por meio do dispositivo VIZIER do Strasbourg Data Center. O catálogo contém dados fotométricos infravermelhos nos comprimentos de onda 3.6, 4.9, 5.8 e $22 \mu \mathrm{m}$, daqui em diante designados como bandas w1, w2, w3 e w4, respectivamente. Em um primeiro passo, selecionamos todos os objetos situados na área que exploramos ao redor de N10, em um intervalo de coordenadas Galácticas $13.11^{\circ}<1<13.27^{\circ}$ e $-0.04^{\circ}<\mathrm{b}<0.12^{\circ}$.

Encontramos 565 fontes WISE nessa área. Posteriormente filtramos essa lista de fontes aplicando uma série de critérios de qualidade definidos por KL, chamado de critério "incerteza/sinalruído/qui-quadrado"(uncertainty/signal-to-noise/chi-squared). O objetivo desse critério é evitar regiões no espaço de um desses parâmetros, com uma probabilidade relativamente elevada de falsa entrada de catálogo. Para w3 mantivemos apenas a condição de $S / R>5$. Considera-se que, se uma fonte satisfaz os critérios para ser uma verdadeira fonte em qualquer uma das bandas, existe uma pequena probabilidade de ser falsa e será incluída na lista final. Após esta filtragem, a lista de fontes foi reduzida a 234 entradas, apresentada na Tabela A.1.

O próximo passo foi separar as fontes em Classe I, Classe II, Disco de Transição e objetos restantes. Seguindo o critério de KL, classificamos os objetos estelares jovens de Classe I, Classe II e Discos de Transição, baseando-nos em diagramas cor-cor. As fronteiras dos loci foram determinadas a partir de amostras conhecidas por KL e nos permitiram classificar os YSOs encontrados na direção de N10.

\footnotetext{
${ }^{2}$ Cutri \& IPAC/WISE Science Data Center Team (2011)
} 
De acordo com KL, os objetos Classe I são assim classificados se suas cores corresponderem aos seguintes critérios:

$$
\begin{array}{r}
w 1-w 3>2.0 \\
e \\
w 1-w 2>-0.42 \times(w 2-w 3)+2.2 \\
e \\
e \\
e \\
w 2-w 3<4.5
\end{array}
$$

Os YSOs Classe II foram classificados de acordo com os seguintes critérios de KL:

$$
\begin{array}{r}
w 1-w 2>0.25 \\
e \\
w 1-w 2<-0.9 \times(w 2-w 3)+0.25 \\
e \\
w 1-w 2>0.46 \times(w 2-w 3)-0.9 \\
e \\
w 2-w 3<4.5
\end{array}
$$

Os Discos de Transição foram, por sua vez, classificados da seguinte forma:

$$
\begin{array}{r}
w 3-w 4>1.5 \\
e \\
0.15<w 1-w 2<0.8 \\
e \\
w 1-w 2>0.46 \times(w 2-w 3)-0.9 \\
e \\
w 1 \leq 13.0
\end{array}
$$


As regiões do diagrama que são usadas para classificar os YSOs são definidas pelos loci limitados pelas linhas nas Figuras $4.10 \mathrm{e} 4.11$.

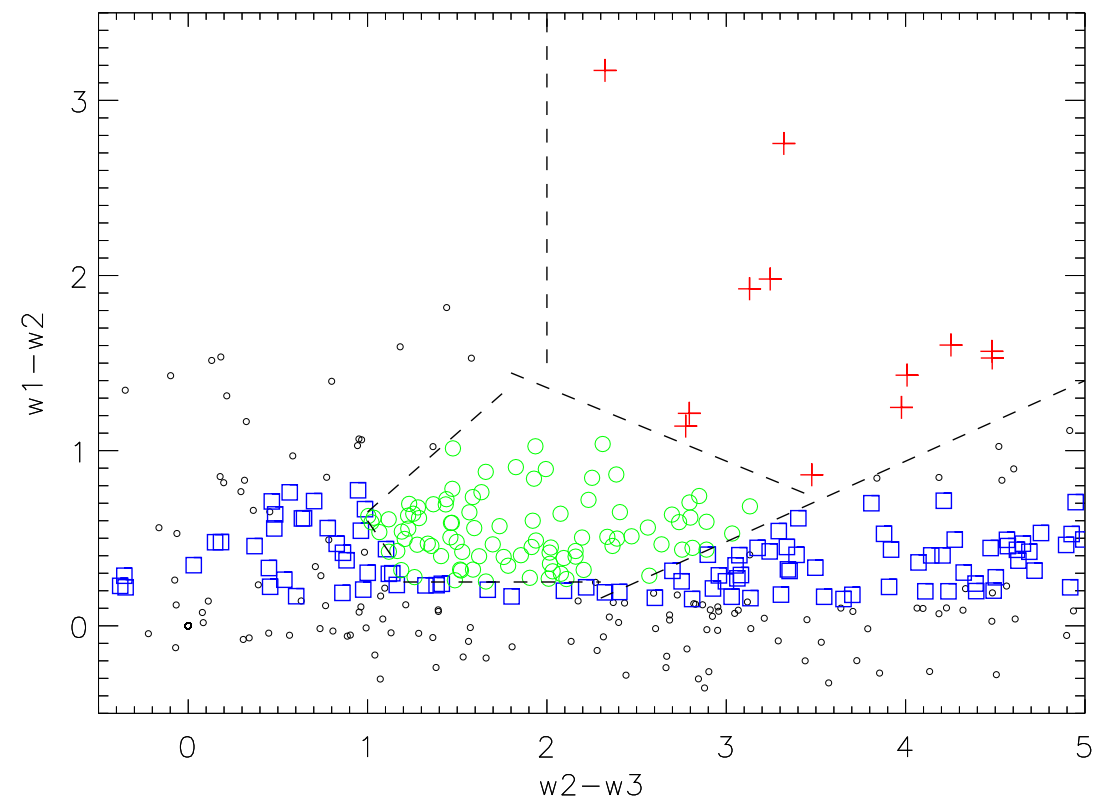

Figura 4.10: Diagrama cor-cor w1-w2 versus w2-w3, de candidatos a YSOs ao redor de N10. Cruzes vermelhas: objetos Classe I; círculos verdes: fontes Classe II; quadrados azuis: Discos de Transição. As linhas tracejadas indicam os limites das regiões de acordo com KL. Os Discos de Transição não são classificados definitivamente neste diagrama. Os objetos restantes são indicados por pontos pretos.

Encontramos 12 objetos Classe I e 91 fontes Classe II. Os Discos de Transição são selecionados separadamente por meio do diagrama cor-cor w1-w2 versus w2-w3 ilustrado na Figura 4.11. Nós encontramos 131 Discos de Transição. Perceba que o critério de seleção anterior segue uma ordem de prioridade: um YSO Classe I continuará sendo Classe I mesmo que este também satisfaça os critérios de Classe II, e depois a seleção de fontes Classe II prevalece sobre a seguinte seleção. Isso explica porque encontramos tantos objetos Classe II no locus definido para os Discos de Transição da Figura 4.11: eles foram classificados como Classe II no passo anterior, com base em um diferente diagrama cor-cor.

A seleção de fontes Classe I é bastante robusta, uma vez que esses objetos são bem separados de outras classes nos diagramas cor-cor. Além disso, nós fizemos experimentos com outros esquemas de classificação disponíveis na literatura (usando dados Spitzer, e.g. Gutermuth et al., 2009) e os mesmos objetos Classe I foram recuperados. Por outro lado, a distinção entre objetos Classe II e Discos de Transição é um pouco arbitrário, assim como podemos ver em algumas sobreposições nas Figuras $4.10 \mathrm{e} 4.11$. 


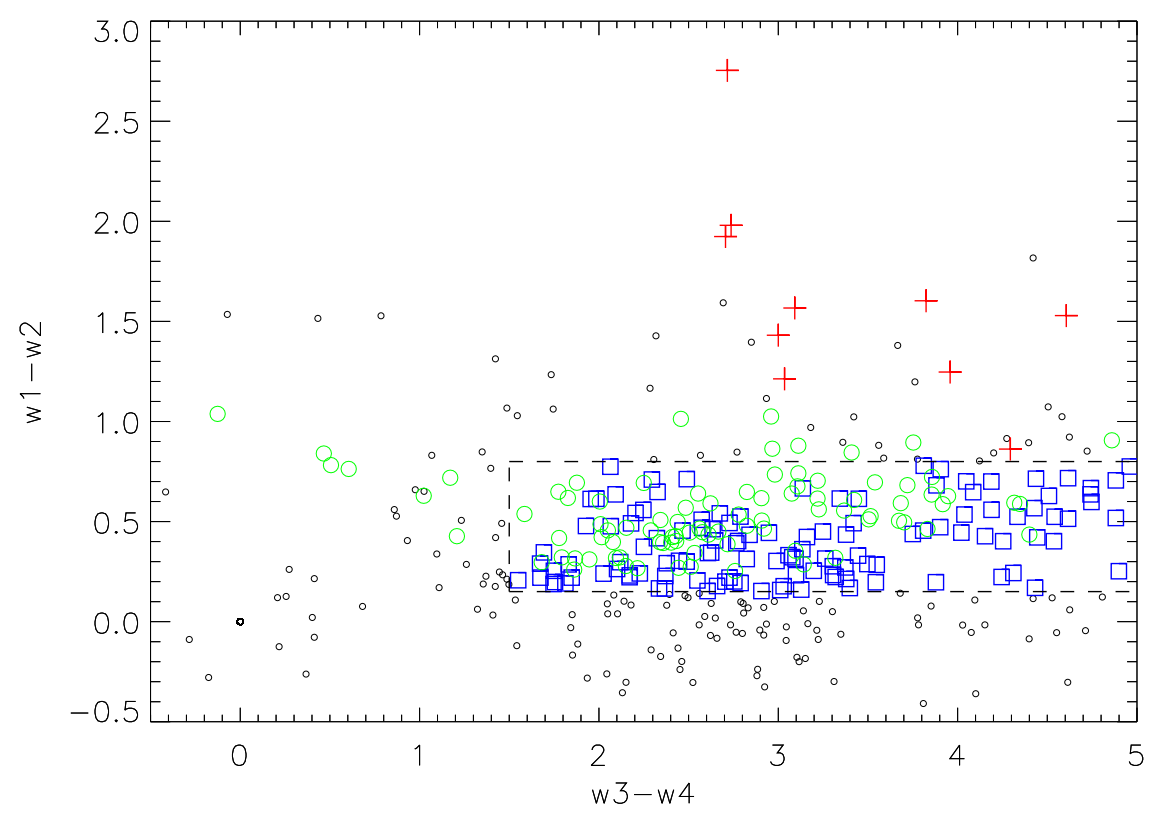

Figura 4.11: Diagrama cor-cor w1-w2 versus w3-w4, parecido com a Figura 4.10 O locus usado para definir os Discos de Transição (quadrados azuis) também contém muitos objetos Classe II (círculos verdes), pois esses objetos foram classificados como Classe II no passo anterior que utiliza outro diagrama cor-cor (Fig. 4.10).

Em amostras de objetos previamente conhecidos como pertencentes a essas dadas classes, usadas por KL para decidir as linhas de fronteira dos loci nas Figuras 4.10 e 4.11 , pode-se ver uma série de Discos de Transição no locus de Classe II e vice-versa. Portanto, devemos considerar que a decisão de classificar as fontes como sendo de uma ou outra classe só é válida no sentido estatístico, sendo correta em cerca de $70 \%$ dos casos. 


\subsection{Ajustes de SED}

Os estágios evolutivos dos YSOs podem ser caracterizados pela distribuição da energia de sua poeira circunstelar. A radiação da estrela central é absorvida por essa poeira e reemitida em outros comprimentos de onda. As diferentes contribuições da emissão da poeira resulta em diferentes SEDs.

Nós comparamos a posição dos YSOs Classe I, os objetos estelares jovens mais embebidos, com a distribuição de gás molecular e poeira fria, como pode ser visto na Figura 4.12. Concluímos que as fontes classificadas de \#1 a \#9 na Tabela A.1 são as mais prováveis de estar fisicamente relacionadas com N10.

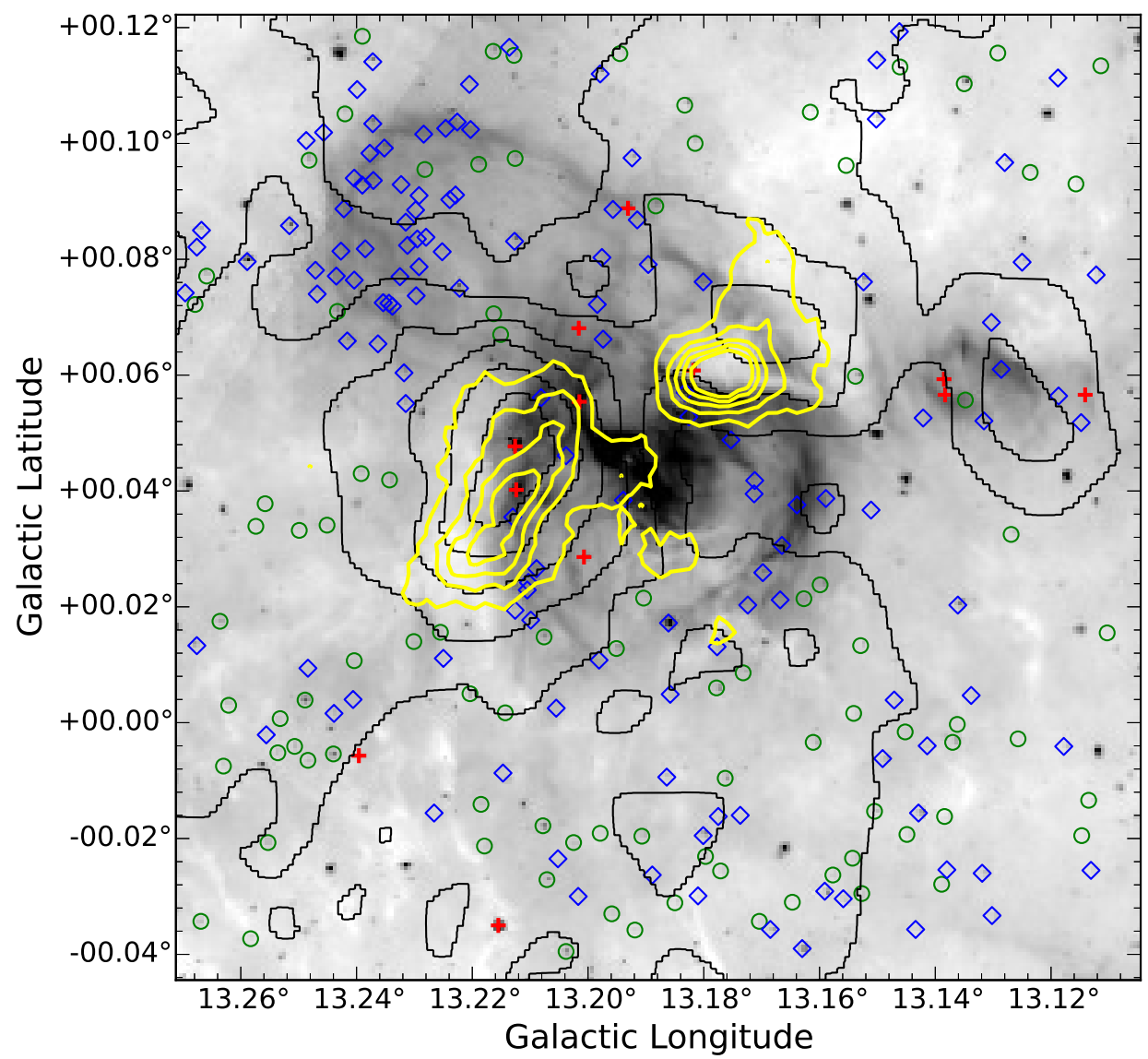

Figura 4.12: Distribuição dos YSOs Classe I (cruzes vermelhas), Classe II (círculos verdes) e Discos de Transição (diamantes azuis). Os contornos em preto mostram a distribuição da componente em $52 \mathrm{~km} \mathrm{~s}^{-1}$ do ${ }^{13} \mathrm{CO}$ e os contornos em amarelo representam a emissão em $870 \mu \mathrm{m}$.

Nós ajustamos as SEDs dos YSOs usando a ferramenta online desenvolvida por Robitaille et al. (2007). Modelos de transferência radiativa são ajustados aos dados observacionais extraídos do catálogo WISE, baseado em teste $\chi^{2}$. Nós selecionamos modelos para os quais: 


$$
\chi^{2}-\chi_{\min }^{2}<3 n
$$

onde $\chi_{\min }^{2}$ é o valor mínimo e $n$ é o número de dados de entrada.

Os ajustes foram feitos utilizando os valores de fluxo nas quatro bandas WISE, com a distância da fonte variando de 4.23 a 5.17 kpc. A extinção interestelar na direção de N10 foi prevista para ser de aproximadamente 10.7 mag de acordo com o modelo S de Amôres \& Lépine (2005), e os valores adotados para a extinção visual variam de 9.7 a 11.7 mag. Os melhores ajustes são mostrados na Figura 4.13. Os valores resultantes para os parâmetros do modelo são fornecidos na Tabela 4.4

De acordo com o melhor modelo ajustado, esses YSOs Classe I tem massa estelar variando de $\sim 1 \mathrm{a} \sim 13 \mathrm{M}_{\odot}$, temperatura estelar $\sim 4000-20000 \mathrm{~K}$, luminosidade total $\sim 3 \times 10^{1}-1 \times 10^{3}$ $\mathrm{L}_{\odot}$, taxa de acreção do envoltório $\sim 9 \times 10^{-8}-3 \times 10^{-3} \mathrm{M}_{\odot} \operatorname{anos}^{-1}$, massa do disco $\sim 7 \times 10^{-3}$ $6 \times 10^{-1} \mathrm{M}_{\odot}$ e idades estelares de $\sim 2 \times 10^{3} \mathrm{a} \sim 1 \times 10^{6}$ anos.
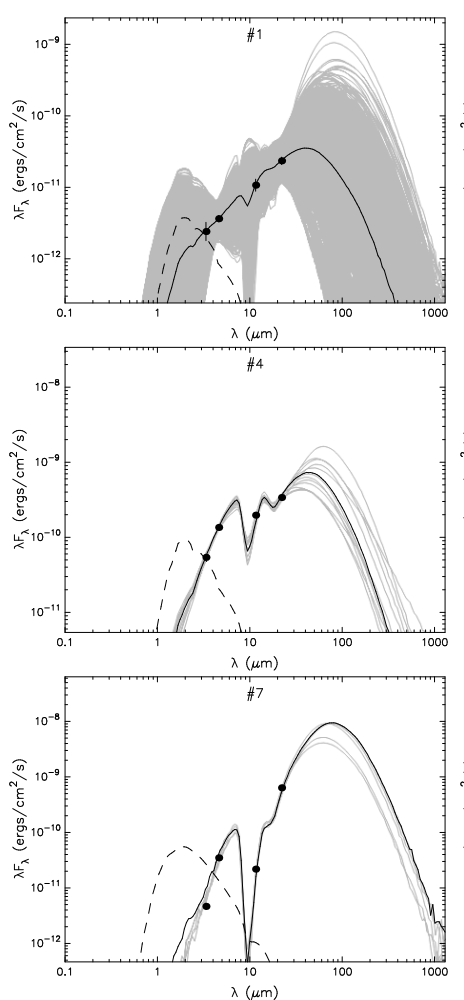
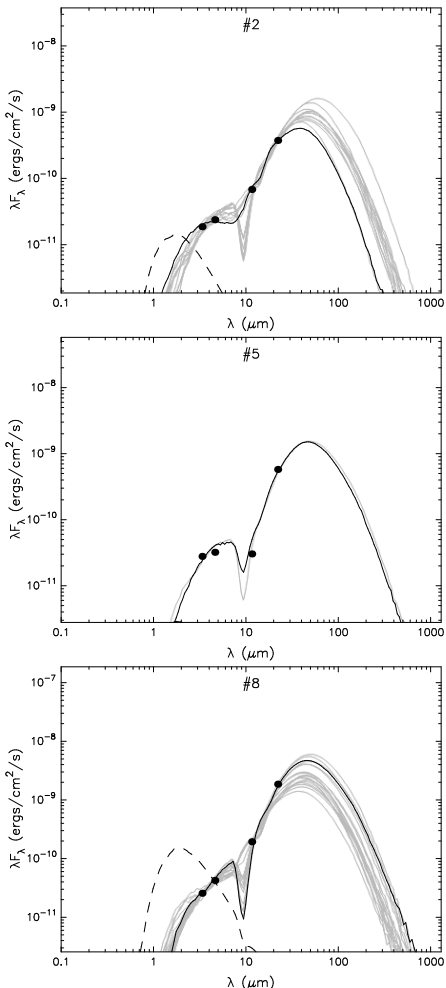
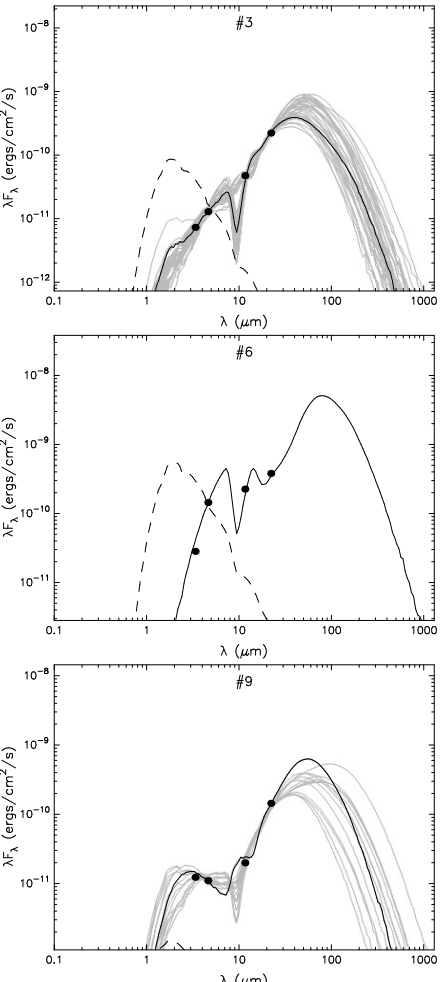

Figura 4.13: SEDs ajustadas para YSOs Classe I na região de N10. Os círculos preenchidos mostram os fluxos de entrada. A linha preta mostra o melhor ajuste. A linha pontilhada mostra a fotosfera estelar correspondente à fonte central de acordo com o modelo de melhor ajuste, como seria na ausência de poeira circunstelar (mas incluindo a extinção interestelar). 


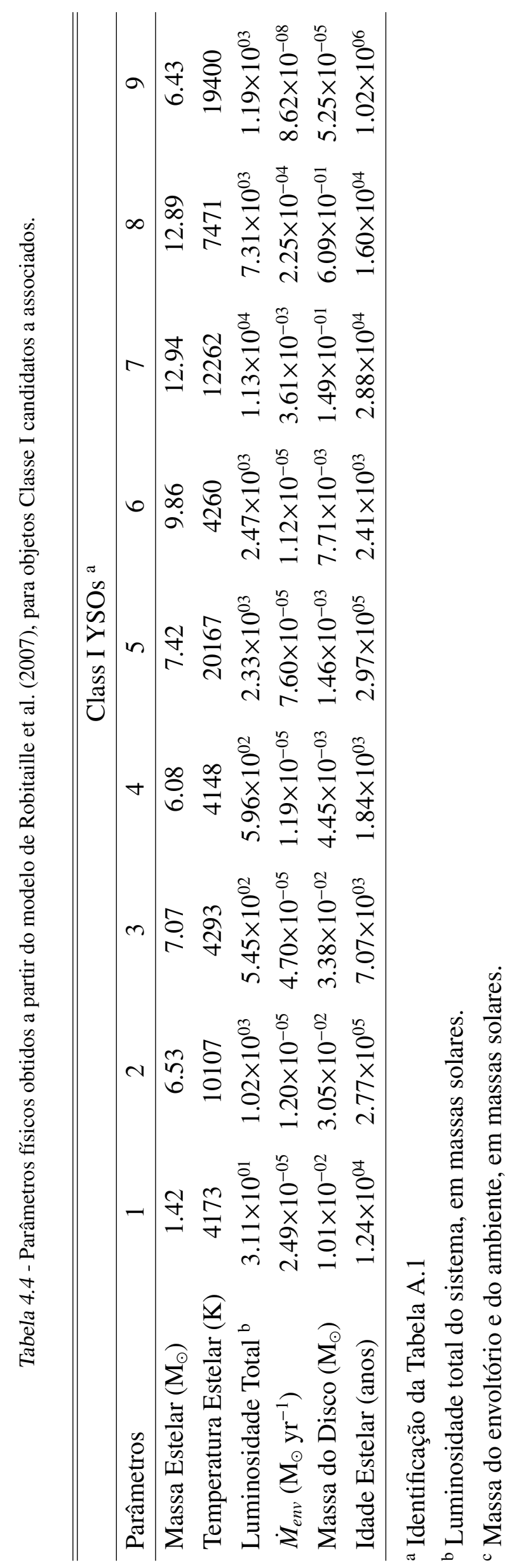




\subsection{Resultados para observações no IRAM 30-m}

As observações realizadas no telescópio IRAM 30-m nos permitiram obter mapas de intensidade integrada e, consequentemente, avaliar a distribuição de determinadas moléculas traçadoras de formação estelar na direção da bolha N10. Realizamos também um levantamento de linha espectral cobrindo a banda de $3 \mathrm{~mm}$ e identificamos diversas moléculas. A seguir apresentamos os resultados obtidos a partir dessas observações.

\subsubsection{Mapas On-The-Fly}

A partir dos nossos dados escolhemos espécies moleculares traçadoras de formação estelar que caracterizam a região. A Tabela 4.5 lista as espécies moleculares, as transições, as frequências e as condições físicas que queremos explorar nessa seção.

Tabela 4.5 - Espécies moleculares detectadas na direção da bolha N10.

\begin{tabular}{c|c|c|c}
\hline \hline Espécie & Transição & Frequência & Condições Físicas \\
\hline $\mathrm{HCO}^{+}$ & $(1-0)$ & $89.188 \mathrm{GHz}$ & ionização \\
$\mathrm{HCN}$ & $(1-0)$ & $88.631 \mathrm{GHz}$ & PDR \\
$\mathrm{SiO}$ & $(2-1)$ & $86.846 \mathrm{GHz}$ & região de choque \\
$\mathrm{N}_{2} \mathrm{H}^{+}$ & $(1-0)$ & $93.173 \mathrm{GHz}$ & cores pré-estelares \\
$\mathrm{CS}$ & $(3-2)$ & $149.969 \mathrm{GHz}$ & gás denso \\
\hline
\end{tabular}

A ferramenta VIEW, do software GreG/GILDAS, nos permitiu checar a qualidade da redução de dados, que apresentamos nas Figuras de 4.14 a 4.18 . 


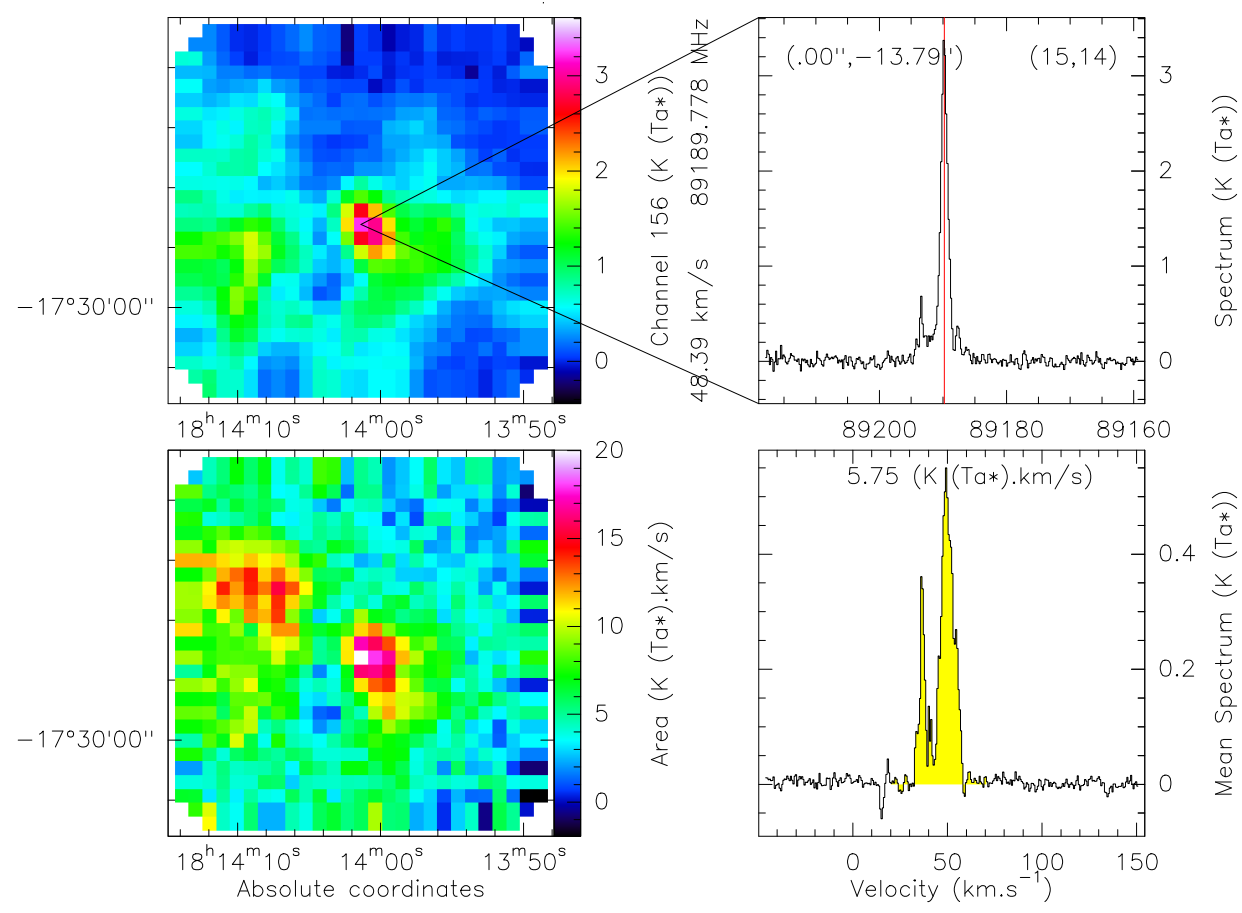

Figura 4.14: Análise preliminar da detecção de $\mathrm{HCO}^{+}(1-0)$ em coordenadas equatoriais. O painel inferior esquerdo mostra o mapa de intensidade integrada de 30 a $70 \mathrm{~km} \mathrm{~s}^{-1}$; o espectro médio da região correspondente à intensidade integrada de $30 \mathrm{a} 70 \mathrm{~km} \mathrm{~s}^{-1}$ encontra-se no painel inferior direito. $\mathrm{O}$ painel superior direito mostra o espectro no pico de emissão identificado no mapa de intensidade integrada; o painel superior esquerdo mostra o mapa de intensidade que corresponde apenas àquele canal, de acordo com a velocidade do pico de emissão.

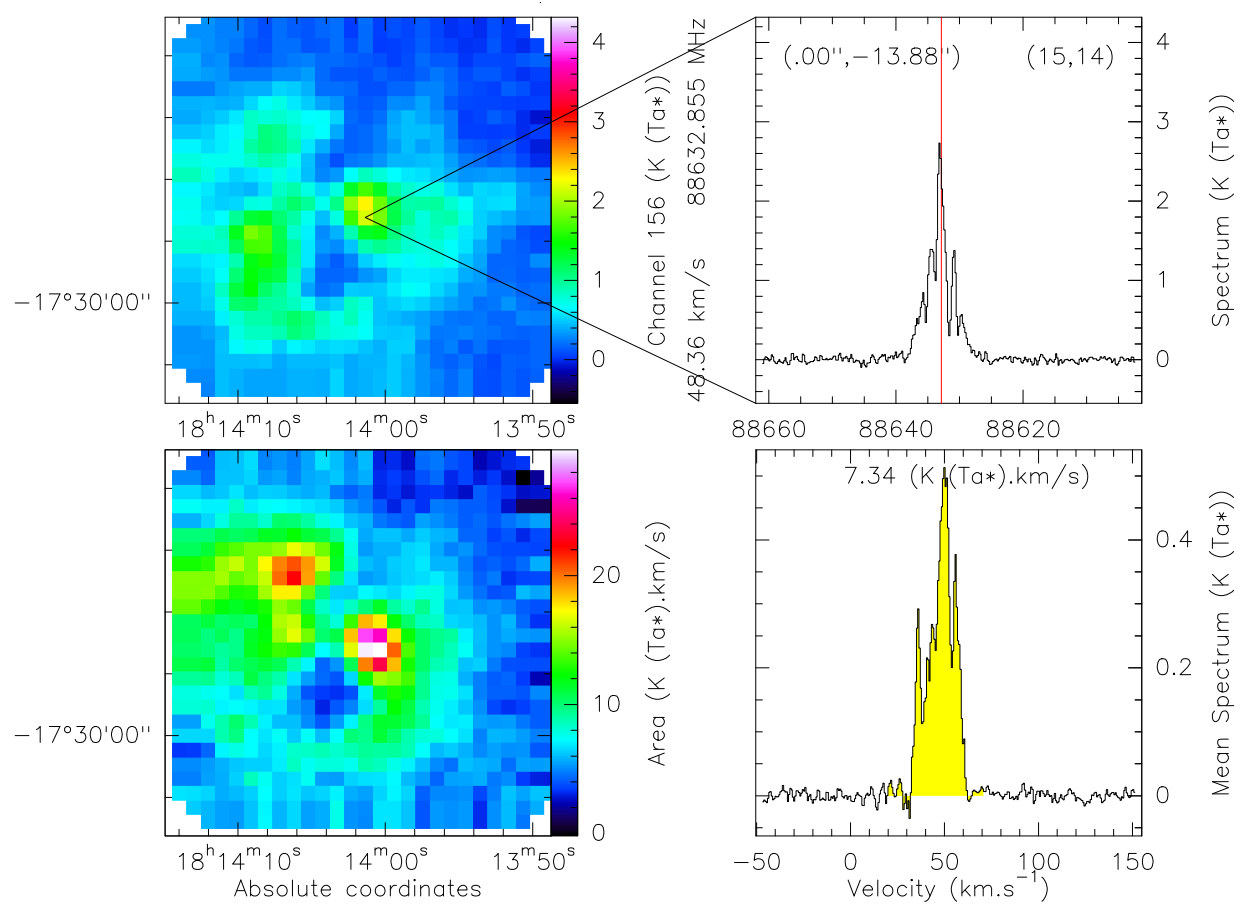

Figura 4.15: O mesmo que a Figura 4.14 para a transição HCN (1 - 0). 


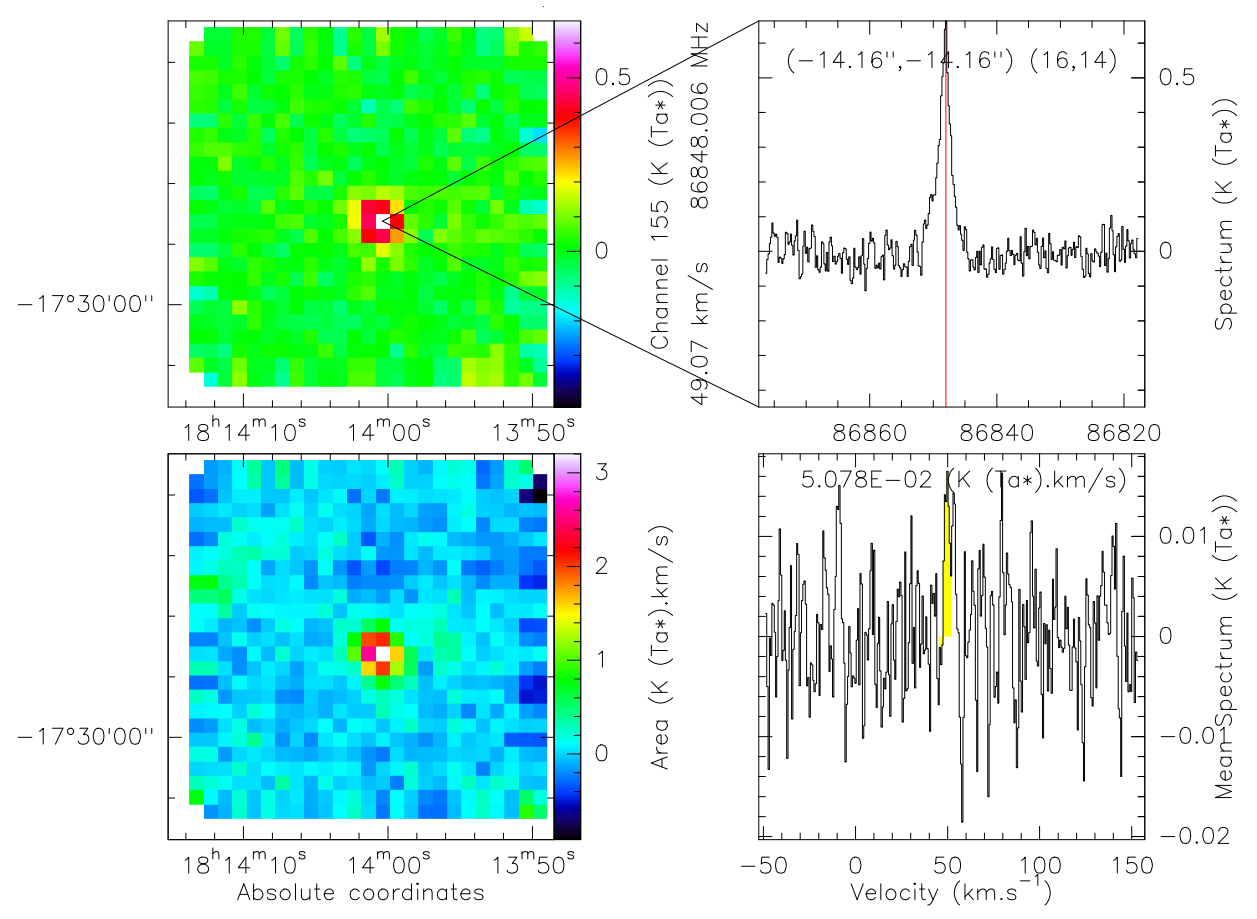

Figura 4.16: O mesmo que a Figura 4.14 para a transição SiO (2 - 1).

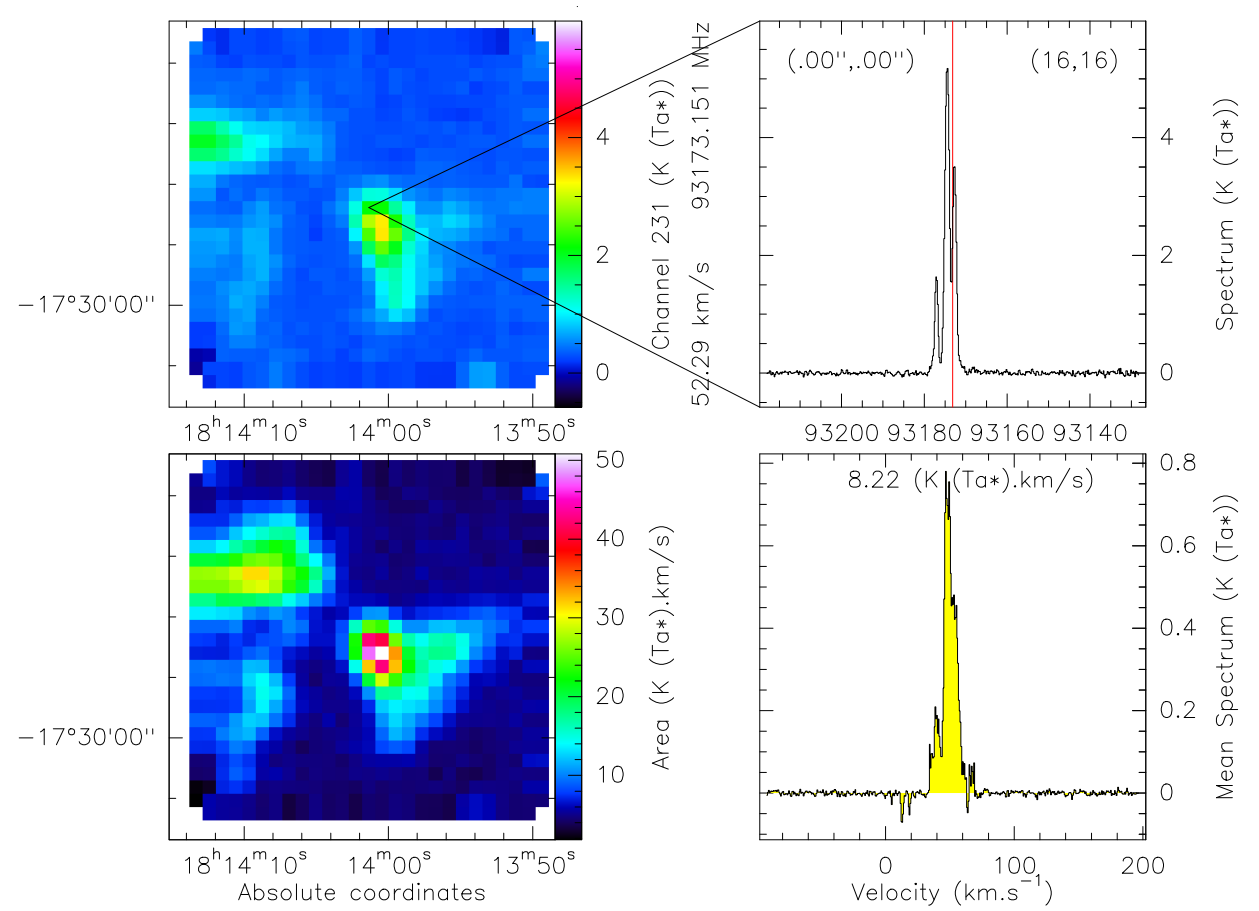

Figura 4.17: O mesmo que a Figura 4.14 para a transição $\mathrm{N}_{2} \mathrm{H}+(1-0)$. 


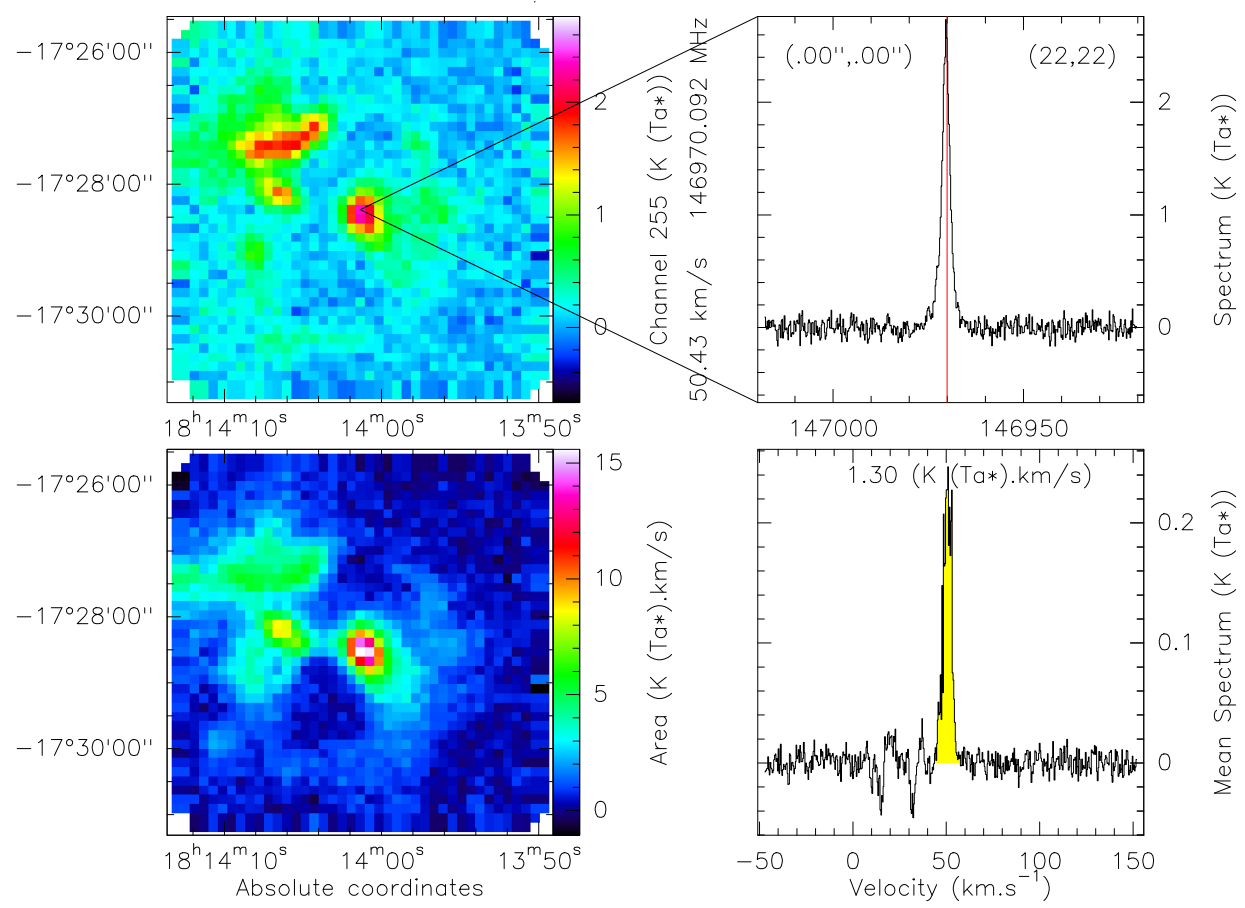

Figura 4.18: O mesmo que a Figura 4.14 para a transição CS (3 - 2). 
Obtivemos mapas de intensidade integrada das transições $\mathrm{HCO}^{+}(1-0), \mathrm{HCN}(1-0), \mathrm{SiO}$ $(2-1), \mathrm{N}_{2} \mathrm{H}+(1-0)$ e CS $(3-2)$, apresentados respectivamente nas Figuras de $4.19 \mathrm{a} 4.23$. Para cada linha molecular, a emissão foi integrada de 30 a $70 \mathrm{~km} \mathrm{~s}^{-1}$ e as coordenadas dos mapas foram convertidas de J2000 para coordenadas Galácticas. A barra de cor indica a temperatura de antena, em Kelvin. O círculo em preto representa o tamanho do feixe para cada frequência observada.

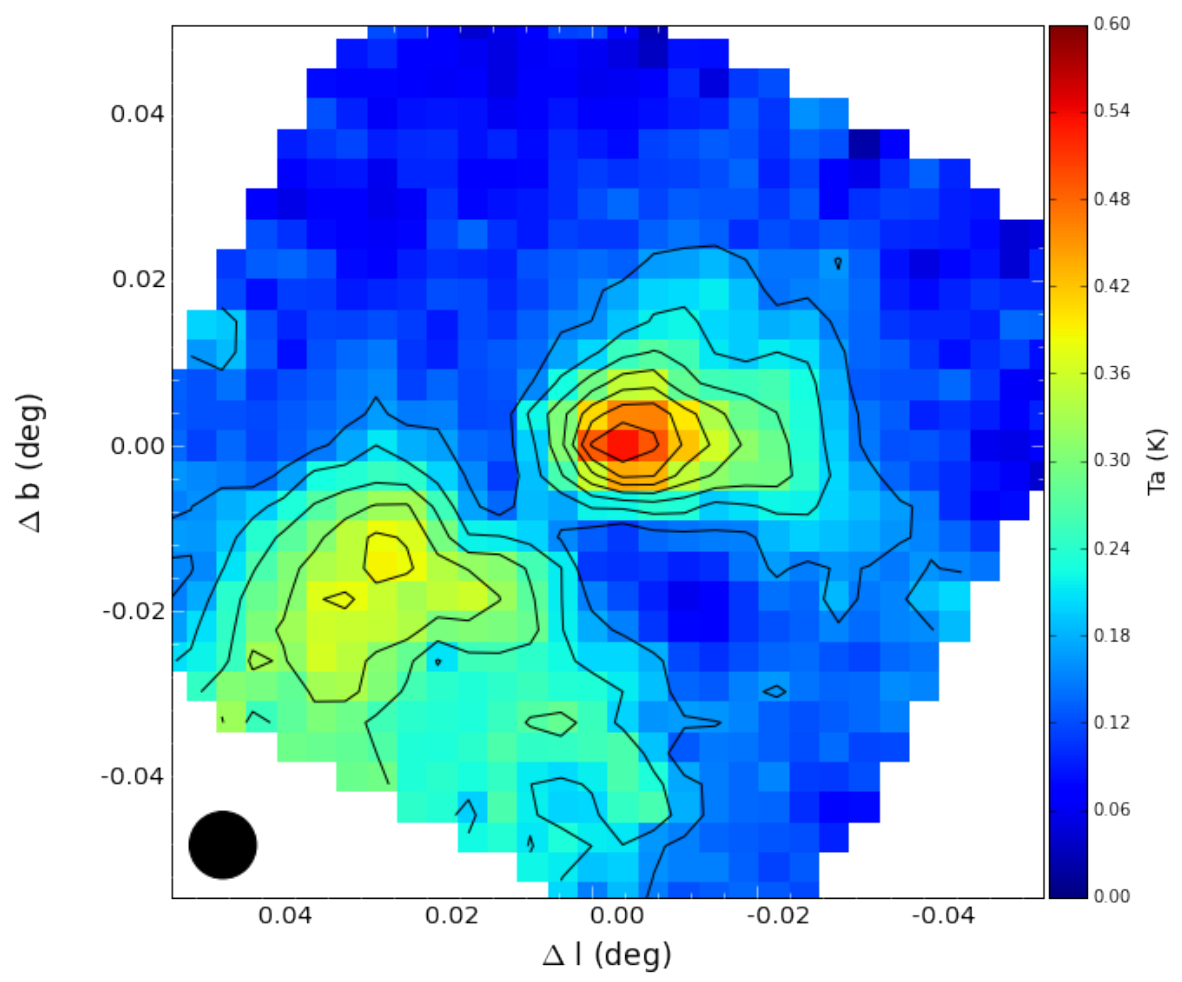

Figura 4.19: Distribuição espacial de $\mathrm{HCO}^{+}(1-0)$ na direção da bolha N10 com contornos de $30 \%$ a $90 \%$ do pico de intensidade $\left(T_{\text {peak }}=1.14 \mathrm{~K}\right)$ em passos de $10 \%$ de $T_{\text {peak }}$. 


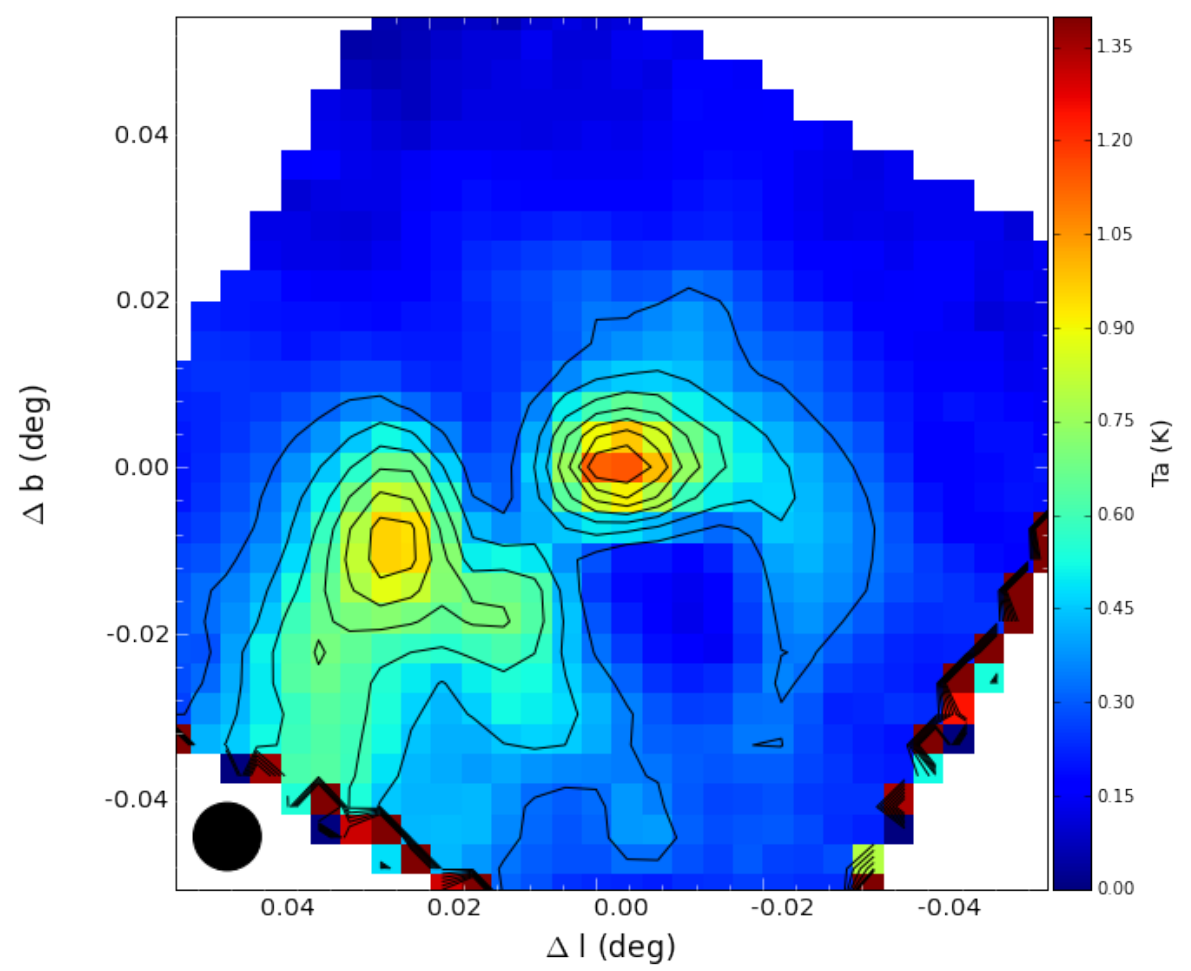

Figura 4.20: Distribuição espacial de HCN (1 - 0) na direção da bolha N10 com contornos de $30 \%$ a $90 \%$ do pico de intensidade $\left(T_{\text {peak }}=0.53 \mathrm{~K}\right)$ em passos de $10 \%$ de $T_{\text {peak }}$.

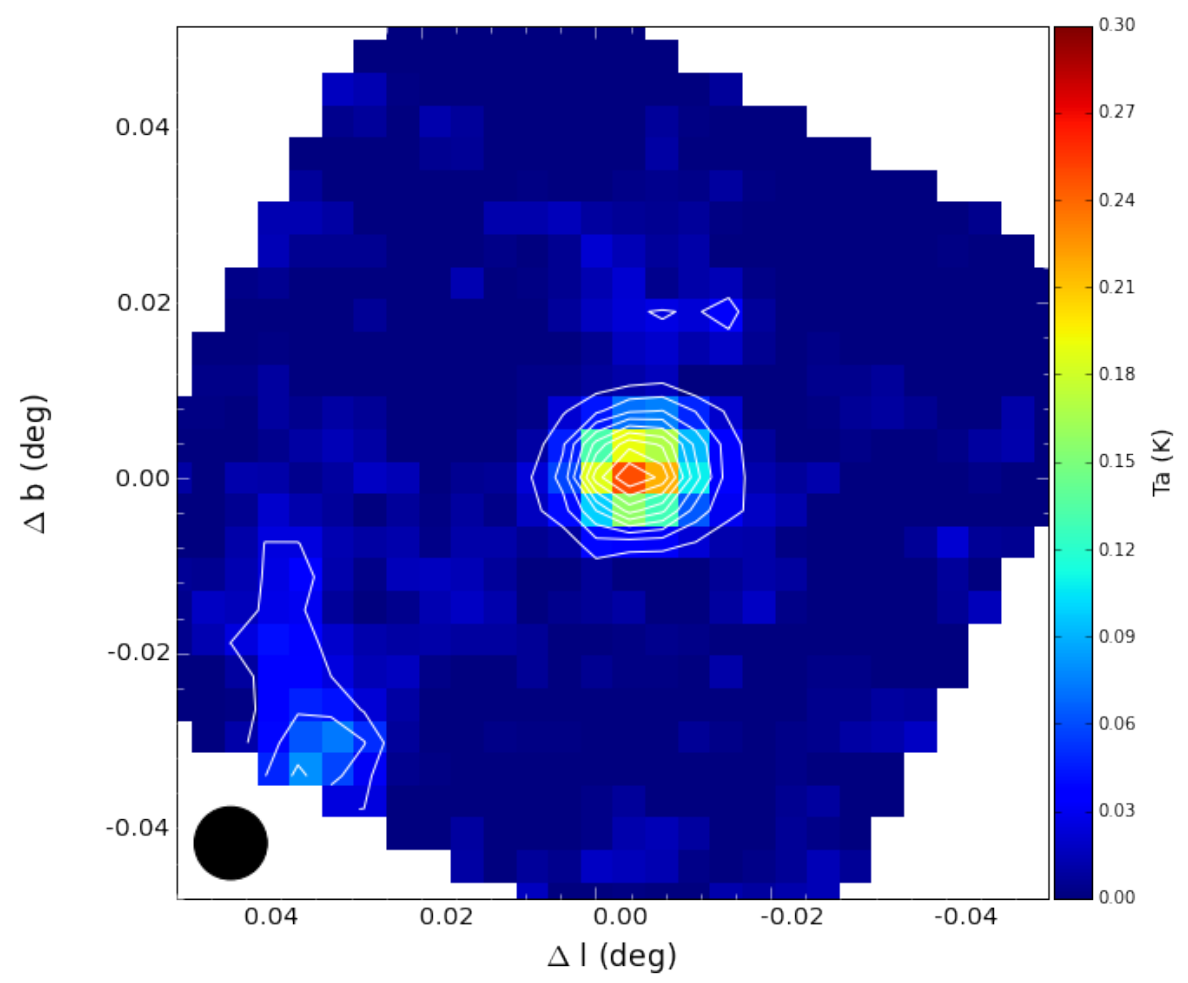

Figura 4.21: Distribuição espacial de $\mathrm{SiO}(2$ - 1) na direção da bolha N10 com contornos de $10 \%$ a $90 \%$ do pico de intensidade $\left(T_{\text {peak }}=0.25 \mathrm{~K}\right)$ em passos de $10 \%$ de $T_{\text {peak }}$. 


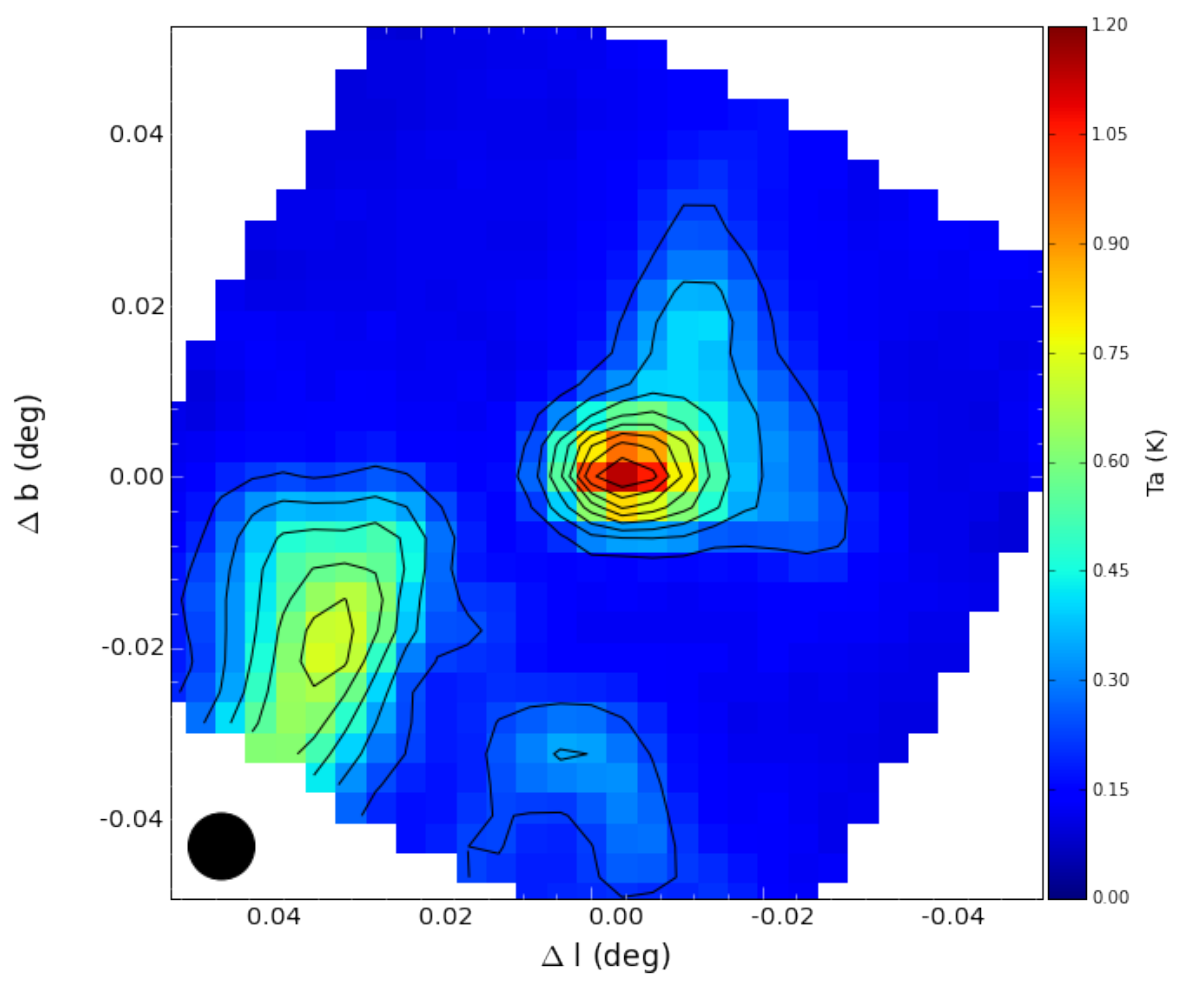

Figura 4.22: Distribuição espacial de $\mathrm{N}_{2} \mathrm{H}+(1-0)$ na direção da bolha $\mathrm{N} 10$ com contornos de $20 \%$ a $90 \%$ do pico de intensidade $\left(T_{\text {peak }}=1.14 \mathrm{~K}\right) \mathrm{em}$ passos de $10 \%$ de $T_{\text {peak }}$.

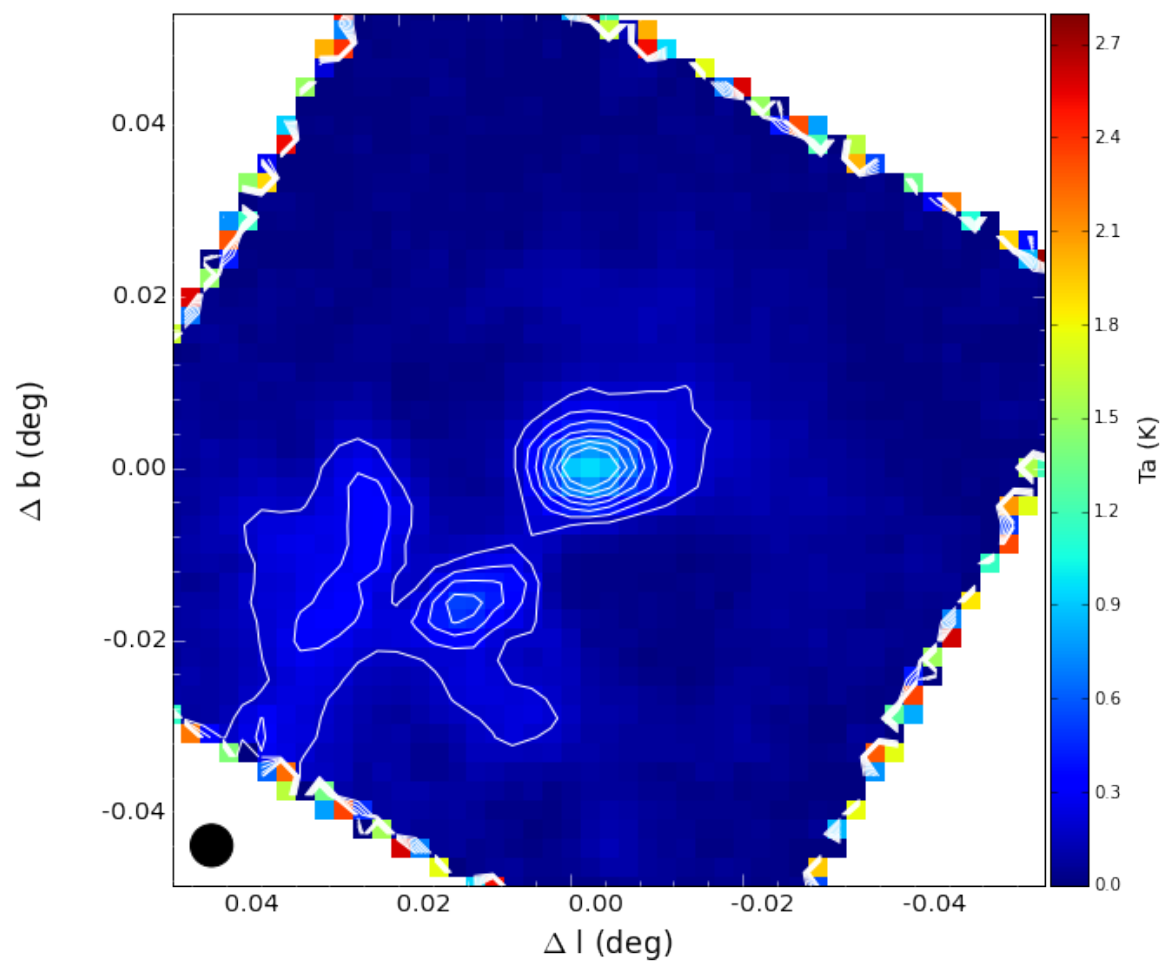

Figura 4.23: Distribuição espacial de CS (3 - 2) na direção da bolha N10 com contornos de $20 \%$ a $90 \%$ do pico de intensidade $\left(T_{\text {peak }}=0.96 \mathrm{~K}\right)$ em passos de $10 \%$ de $T_{\text {peak }}$. 


\subsubsection{Outflow}

A presença de emissão $\mathrm{SiO}$ e asas extendidas de $\mathrm{HCO}^{+}$é uma evidência de atividade de outflow na nuvem (Vasyunina et al., 2011). A Figura 4.24 mostra a distribuição de $\mathrm{HCO}^{+}(1-0)$ e $\mathrm{SiO}(2-1)$ com relação à bolha infravermelha N10.

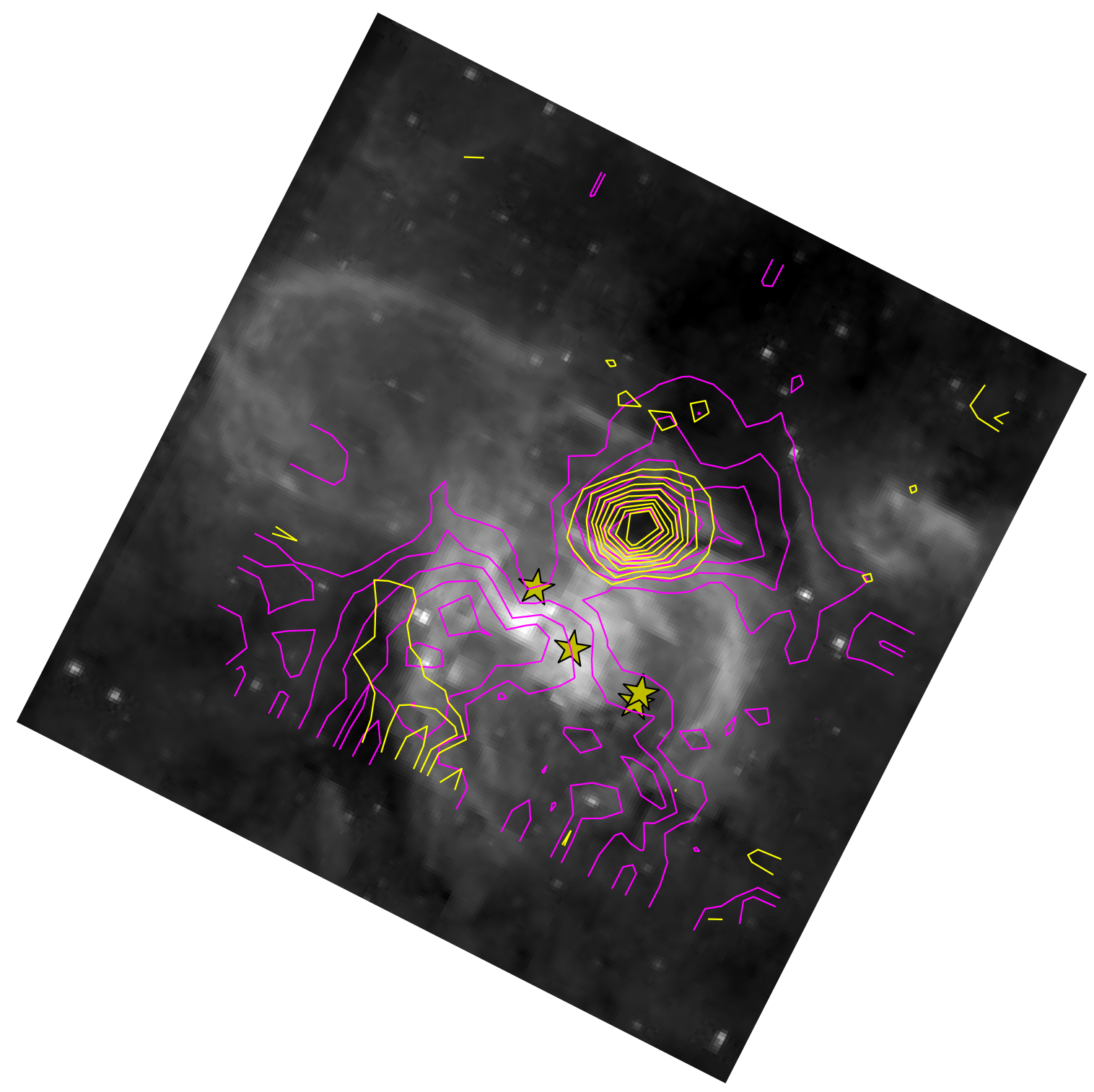

Figura 4.24: Mapa da distribuição de $8.0 \mu \mathrm{m}$, em escala de cinza. Os contornos em magenta, indicando a distribuição de $\mathrm{HCO}^{+}(1-0)$, são os mesmos da Figura 4.19 Os contornos em amarelo mostram a emissão de $\mathrm{SiO}(2-1)$, em passos iguais aos da Figura 4.23 . As estrelas são as candidatas a fontes ionizantes citadas na Tabela 2.1

A emissão de $\mathrm{HCO}^{+}$é mais extendida, enquanto $\mathrm{SiO}$ é compacta. Entretanto, o pico de emissão das duas moléculas coincidem na posição de maior densidade, também traçada pela emissão da poeira fria em $870 \mu \mathrm{m}$ (veja painel inferior da Figura 4.6 e Figura 4.7). A emissão de $\mathrm{HCO}^{+}(1-0)$ indica a presença de radiação UV ionizante, provinda da(s) estrela(s) no centro de 
N10. O pico das emissões de $\mathrm{SiO}$ e $\mathrm{HCO}+$ parecem localizar-se preferencialmente entre a casca molecular e a região HII, como mostra a Figura 4.24

A Figura 4.25 mostra os espectros de $\mathrm{HCO}^{+}(1-0)$ e $\mathrm{SiO}(2-1)$ na direção do pico de emissão.
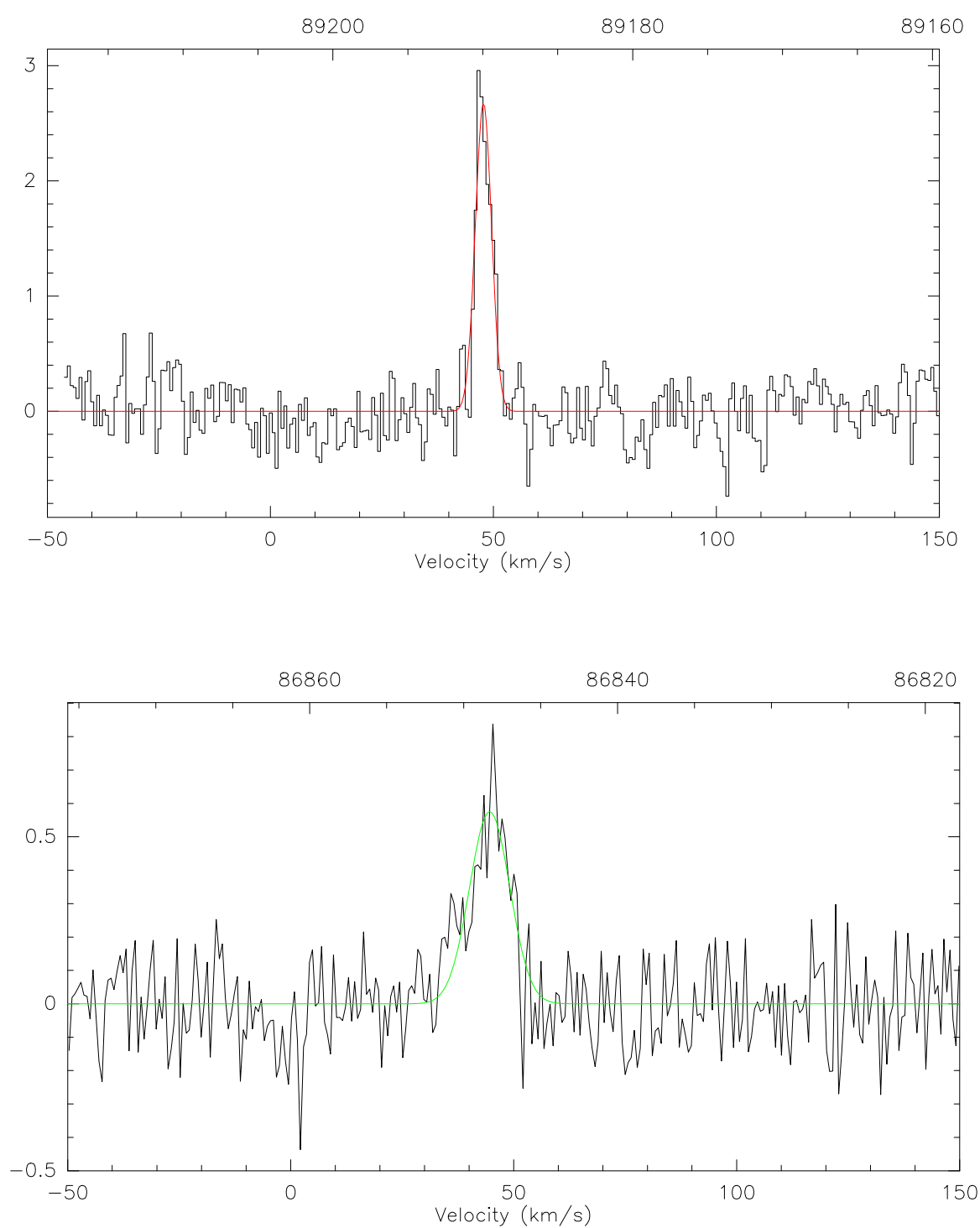

Figura 4.25: Painel superior: perfil de linha de $\mathrm{HCO}^{+}(1-0)$. Painel inferior: perfil de linha de $\mathrm{SiO}(2-1)$. Ambos na direção do pico de emissão, com intensidade em Ta (K).

$\mathrm{HCO}^{+}(1-0)$ exibe um espectro com uma componente centrada em $V_{l s r} \sim 48 \mathrm{~km} \mathrm{~s}^{-1}$ com um pico de intensidade de $2.7 \mathrm{~K}$ e largura de linha $\Delta V \sim 4.3 \mathrm{~km} \mathrm{~s}^{-1}$. O espectro de $\mathrm{SiO}(2-1)$ exibe uma única componente centrada em $V_{l s r} \sim 45 \mathrm{~km} \mathrm{~s}^{-1}$, com um pico de intensidade de 0.6 $\mathrm{K}$ e $\Delta V \sim 10.9 \mathrm{~km} \mathrm{~s}^{-1}$, ou seja, mais alargada que a componente $\mathrm{HCO}^{+}(1-0)$. Um perfil que mostra evidências de alargamento é esperado em um gás perturbado pela frente de choque na região. Os dois perfis apresentam um pico deslocado para o azul com respeito à velocidade do 
sistema, $54.1 \mathrm{~km} \mathrm{~s}^{-1}$ (vide Tabela 4.1), indicando que essas componentes se encontram entre nós e a região HII.

\subsubsection{Integração profunda}

A riqueza química de uma das nuvens moleculares em N10 foi confirmada pelo levantamento de linhas espectrais realizada na direção da condensação molecular mais brilhantes de N10. A Figura 4.26 mostra o levantamento espectral sensível e as espécies moleculares identificadas são listadas na Tabela 4.6. A Tabela 4.7 classifica as moléculas que identificamos, de acordo com suas propriedades.

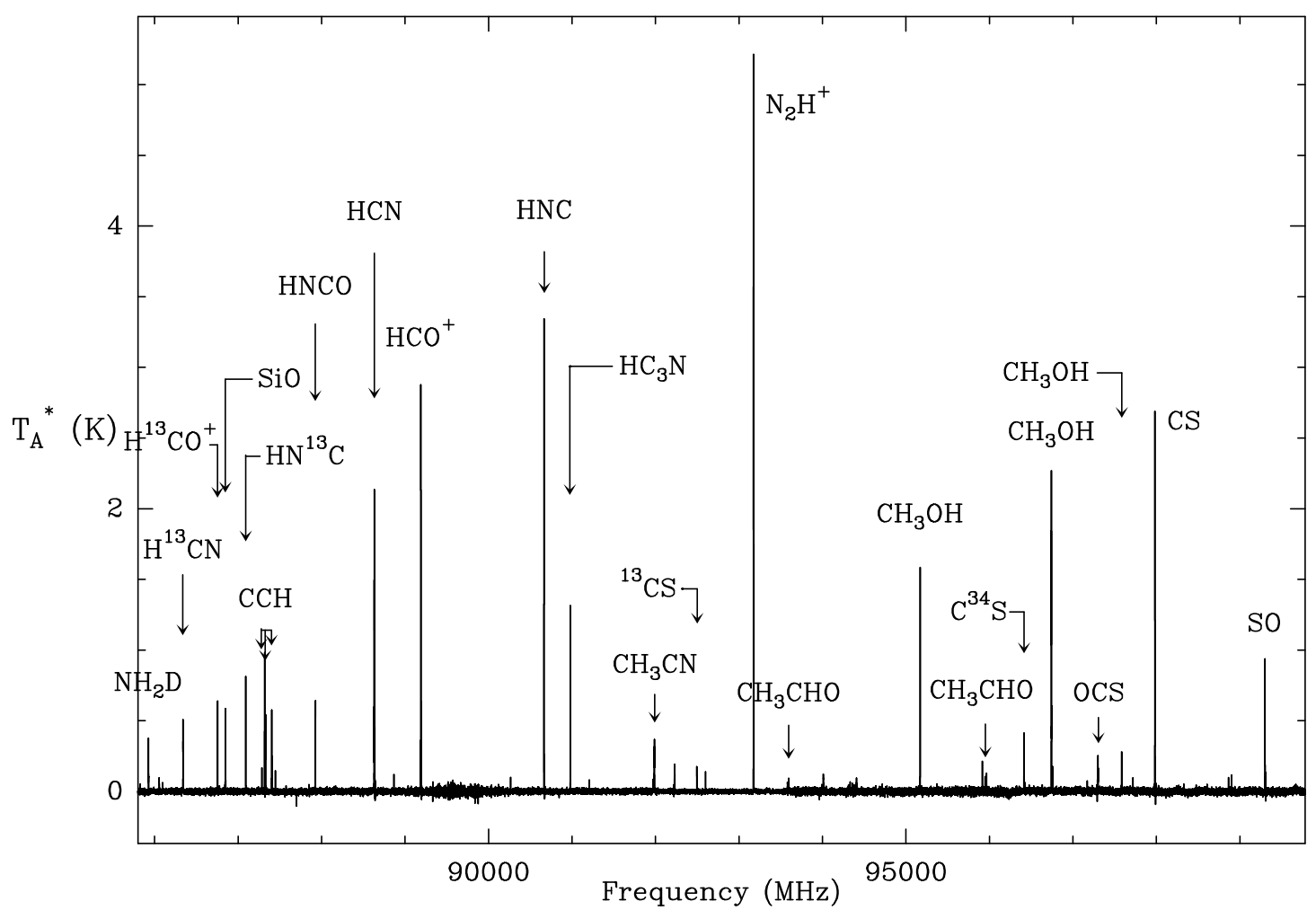

Figura 4.26: Espectro de integração profunda na direção da condensação molecular mais densa de N10. 
Tabela 4.6 - Conteúdo molecular detectado no levantamento de linhas espectrais.

\begin{tabular}{|c|c|c|}
\hline Espécie & Número de linhas detectadas & Observações \\
\hline $\mathrm{NH}_{2} \mathrm{D}$ & 4 & estrutura hiperfina \\
\hline $\mathrm{HC}^{15} \mathrm{~N}$ & 1 & \\
\hline SO & 1 & \\
\hline $\mathrm{H}^{13} \mathrm{CN}$ & 1 & \\
\hline $\mathrm{HCO}$ & 3 & estrutura hiperfina \\
\hline $\mathrm{H}^{13} \mathrm{CO}^{+}$ & 1 & \\
\hline $\mathrm{SiO}$ & 1 & \\
\hline $\mathrm{HN}^{13} \mathrm{C}$ & 1 & \\
\hline $\mathrm{CCH}$ & 6 & \\
\hline $\mathrm{NH}_{2} \mathrm{CHO}$ & 3 & \\
\hline $\mathrm{HNCO}$ & 2 & \\
\hline $\mathrm{HCN}$ & 1 & \\
\hline $\mathrm{H}^{15} \mathrm{NC}$ & 1 & \\
\hline $\mathrm{HCO}^{+}$ & 1 & \\
\hline${ }^{15} \mathrm{NNH}^{+}$ & 3 & \\
\hline $\mathrm{HNC}$ & 1 & \\
\hline $\mathrm{CH}_{3} \mathrm{CN}$ & 10 & \\
\hline${ }^{13} \mathrm{CS}$ & 1 & \\
\hline $\mathrm{NNH}^{+}$ & 3 & estrutura hiperfina \\
\hline $\mathrm{CH}_{3} \mathrm{CHO}$ & 14 & \\
\hline${ }^{13} \mathrm{CH}_{3} \mathrm{OH}$ & 5 & \\
\hline $\mathrm{CH}_{3} \mathrm{OH}$ & 16 & \\
\hline $\mathrm{C}^{34} \mathrm{~S}$ & 2 & \\
\hline $\mathrm{HC}_{3} \mathrm{~N}$ & 3 & \\
\hline OCS & 3 & \\
\hline $\mathrm{CS}$ & 2 & \\
\hline SO & 2 & \\
\hline${ }^{13} \mathrm{CN}$ & 8 & estrutura hiperfina \\
\hline $\mathrm{C}^{18} \mathrm{O}$ & 1 & \\
\hline
\end{tabular}


Tabela 4.6 - continua

\begin{tabular}{lcc}
\hline \hline Espécie & Número de linhas detectadas & Observações \\
\hline${ }^{13} \mathrm{CO}$ & 1 \\
$\mathrm{C}^{17} \mathrm{O}$ & 1 \\
$\mathrm{CN}$ & 9 \\
$\mathrm{NS}$ & 1 \\
$\mathrm{CO}$ & 1 \\
$\mathrm{H}_{2} \mathrm{CS}$ & 1 \\
$\mathrm{H}_{2} \mathrm{CCO}$ & 2 \\
$\mathrm{H}_{2} \mathrm{CO}$ & 1 \\
$\mathrm{H}_{2} 2^{13} \mathrm{CO}$ & 2 \\
$\mathrm{DCO}^{+}$ & 1 \\
$\mathrm{DCN}^{\mathrm{c}-\mathrm{C}_{3} \mathrm{H}_{2}}$ & 1 \\
$\mathrm{C}^{33} \mathrm{~S}$ & 1 \\
\hline
\end{tabular}


Tabela 4.7 - Classificação das moléculas detectadas de acordo com suas propriedades.

Moléculas orgânicas complexas

$\begin{array}{llll}\mathrm{NH}_{2} \mathrm{CHO} & \mathrm{CH}_{3} \mathrm{CHO} & { }^{13} \mathrm{CH}_{3} \mathrm{OH} \quad \mathrm{CH}_{3} \mathrm{OH}\end{array}$

Moléculas que contêm nitrogênio

\begin{tabular}{lllll}
$\mathrm{HC}^{15} \mathrm{~N}$ & $\mathrm{~N} 2 \mathrm{HD}$ & $\mathrm{H}^{13} \mathrm{CN}$ & $\mathrm{HN}^{13} \mathrm{C}$ & $\mathrm{HNCO}$ \\
$\mathrm{HCN}$ & $\mathrm{H}^{15} \mathrm{CN}$ & ${ }^{15} \mathrm{NNH}$ & $\mathrm{HCN}$ & $\mathrm{CH}_{3} \mathrm{CN}$ \\
$\mathrm{HC}_{3} \mathrm{~N}$ & ${ }^{13} \mathrm{CN}$ & $\mathrm{CN}$ & $\mathrm{DCN}$ & \\
\hline
\end{tabular}

Moléculas que contêm enxofre

\begin{tabular}{|c|c|c|c|c|}
\hline SO & ${ }^{13} \mathrm{CS}$ & $C^{34} S$ & OCS & $\mathrm{CS}$ \\
\hline NS & $\mathrm{H}_{2} \mathrm{CS}$ & $\mathrm{C}^{33} \mathrm{~S}$ & & \\
\hline \multicolumn{5}{|c|}{ Íons moleculares } \\
\hline $\mathrm{HCO}^{+}$ & $\mathrm{NNH}^{+}$ & $\mathrm{DCO}^{+}$ & & \\
\hline \multicolumn{5}{|c|}{ Outras moléculas } \\
\hline $\mathrm{HCO}$ & $\mathrm{H}^{13} \mathrm{CO}$ & $\mathrm{SiO}$ & $\mathrm{CCH}$ & $\mathrm{C}^{18} \mathrm{O}$ \\
\hline${ }^{13} \mathrm{CO}$ & $\mathrm{C}^{17} \mathrm{O}$ & $\mathrm{CO}$ & $\mathrm{H}_{2} \mathrm{CCO}$ & $\mathrm{H}_{2} \mathrm{CO}$ \\
\hline $\mathrm{H}_{2}^{13} \mathrm{CO}$ & $\mathrm{c}-\mathrm{C}_{3} \mathrm{H}_{2}$ & & & \\
\hline
\end{tabular}




\section{Capítulo 5}

\section{Discussão}

\subsection{Distribuição de $\mathrm{CO}$ ao redor de N10}

As nuvens moleculares são frias $(\sim 10 \mathrm{~K})$ e densas $\left(10^{3} \mathrm{~cm}^{-3}\right)$. Nessas condições as transições rotacionais são as mais facilmente excitadas, como na molécula $\mathrm{CO}$, por exemplo. Embora a espécie $\mathrm{H}_{2}$ seja a mais abundante, ela não possui momento de dipolo elétrico permanente e portanto não apresenta linhas rotacionais.

Já CO é a segunda molécula mais abundante e tem momento de dipólo permanente. Além disso, seu primeiro nível de rotação acima do estado fundamental é de $5 \mathrm{~K}$, facilmente excitado. Nesta seção discutiremos a distribuição do material molecular ao redor da bolha N10, traçado pela molécula $\mathrm{CO}$, e sua conexão com a história de formação estelar na região.

Os mapas de canal são apresentados nas Figuras 4.2 e 4.3 . A transição de ${ }^{12} \mathrm{CO}$ é opticamente espessa, logo sua emissão parece se espalhar por uma área maior, enquanto a molécula ${ }^{13} \mathrm{CO}$ traça regiões mais densas, uma vez que é opticamente mais fina. A Figura 4.5 mostra dois picos de emissão molecular, isso é, duas condensações de ${ }^{13} \mathrm{CO}$. O Clump \#1 está centrado em $l=13.218^{\circ}, b=0.043^{\circ}$ e o Clump \#2 em $l=13.169^{\circ}, b=0.072^{\circ}$. Os dois clumps estão localizados precisamente nas fronteiras da estrutura anelar revelada pela emissão em $8.0 \mu \mathrm{m}$, evidenciada por uma elipse na Figura 2.1 .

A distância por nós estimada está compatível com aquelas encontradas na literatura (e.g. Pandian et al., 2008; Deharveng et al., 2010). Considera-se que N10 está perto da extremidade próxima da barra Galáctica, uma região de formação estelar intensa, como mostraremos na Seção 5.1 .1 


\subsubsection{Outras componentes da emissão em CO}

Como foi destacado na Seção 4.1, as componentes de $\mathrm{CO}$ em 20 e $37 \mathrm{~km} \mathrm{~s}^{-1}$ não parecem estar fisicamente relacionadas com a bolha N10. Qual seria, então, a origem dessa contribuição?

Sabe-se que a emissão de CO

em galáxias espirais está concentrada em seus braços (Nieten et al., 2006; Schinnerer et al. 2013). A linha de visada na direção de N10 atravessa dois braços espirais de nossa Galáxia antes de atingir a distância de 4.7 kpc, como mostra a Figura 5.1.

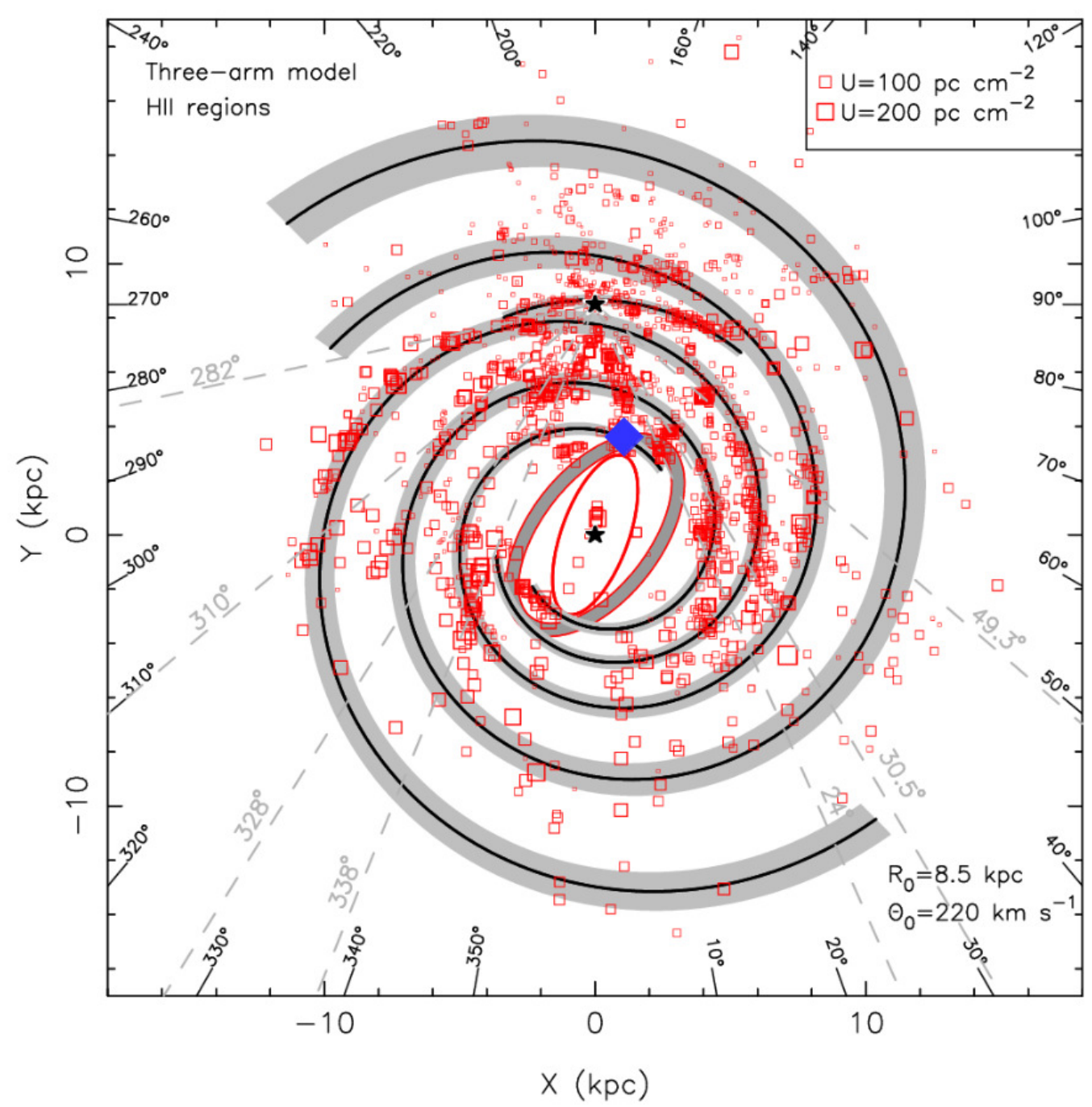

Figura 5.1: Melhor ajuste para um modelo de três braços espirais da Galáxia para a distribuição de regiões HII, representadas pelos quadrados vermelhos. A estrela em preto acima indica a posição do Sol e a outra estrela preta indica o centro da Galáxia. A elipse vermelha aberta indica a barra Galáctica e a elipse preenchida em cinza mostra o melhor ajuste para o Braço 3 kpc Próximo e Braço 3 kpc Distante. As linhas tracejadas indicam as direções tangenciais observadas. O diamante em azul mostra a posição aproximada da bolha N10 no plano da Galáxia. Figura adaptada de Hou \& Han (2014). 


\subsubsection{Situação da formação estelar}

O clump de poeira fria, observado em $870 \mu \mathrm{m}$ e detalhado na Seção 4.2, parece ser um bom candidato a região de formação de aglomerados estelares (clusters). Isso pelo fato dessa condensação apresentar massa total $M_{t o t}=240 \mathrm{M}_{\odot}$ e raio médio $R=0.37$ pc. De acordo com Motte et al. (2003), fragmentos moleculares de 0.09 a 0.56 pc com massas cobrindo um intervalo de 20 até $3600 \mathrm{M}_{\odot}$ têm características de protoaglomerados (protoclusters).

Embora tenhamos encontrado evidências de formação estelar na região de N10, não temos certeza se a formação dos YSOs foi desencadeada pelo mecanismo "Collect and Collapse" ao redor da bolha. A fim de verificar esse processo, podemos aplicar o modelo analítico proposto por Whitworth et al. (1994) e comparamos a escala de tempo de fragmentação $\left(t_{\text {frag }}\right)$ com a idade dinâmica da bolha $\left(t_{d y n}\right)$.

O modelo de Whitworth et al. (1994) descreve o tempo de fragmentação, em anos, como Liu et al. (2016) mostram em sua formulação:

$$
\mathrm{t}_{\text {frag }}=1.56 c_{s}^{7 / 11} N_{u v}^{-1 / 11} n_{o}^{-5 / 11},
$$

onde $c_{s}$ é a velocidade do som isotérmica no gás ionizado em unidades de $0.2 \mathrm{~km} \cdot \mathrm{s}^{-1}, N_{u v}$ é o fluxo de fótons ionizantes em unidades de $10^{49}$ fótons $\mathrm{s}^{-1}$ e $n_{o}$ é a densidade inicial de partículas no ambiente nêutro em unidades de $10^{3} \mathrm{~cm}^{-3}$.

O fluxo de fótons pode ser calculado por meio da relação abaixo (Liu et al., 2015):

$$
N_{u v}=0.76 \times 10^{47} T_{4}^{-0.45} v_{G H z}^{0.1} S_{v} d_{k p c}^{2}
$$

onde $T_{4}$ é a temperatura eletrônica em unidades de $10 \mathrm{~K}, S_{v}$ é a medida da densidade de fluxo em unidades de Jansky, $v_{G H z}$ é a frequência em unidades de Gigahertz e $d_{k p c}$ é a distância em kiloparsec.

Considerando $c_{s}=0.2 \mathrm{~km} \mathrm{~s}^{-1}$ (Liu et al., 2015), $N_{u v}=1.86 \times 10^{49}$ fótons s${ }^{-1}$ e $n_{o}$ da ordem de $\sim 10^{3} \mathrm{~cm}^{-3}$ (Ma et al. 2013), estimamos um valor $t_{\text {frag }} \sim 1.5 \times 10^{6}$ anos para a bolha. Da Seção 4.3 temos que $t_{d y n}=9.17 \times 10^{4}$ anos, ou seja, a idade dinâmica é menor que a escala de tempo de fragmentação, o que sugere que a região não suporta o mecanismo "Collect and Collapse". Nesse caso, o cenário "Radiation-Driven Implosion” deve ser considerado e investigado. 
A Figura 4.12 mostra a distribuição espacial dos YSOs identificados listados na Tabela A.1. A posição dos YSOs Classe I comparada com a distribuição de gás traçado pelo CO e poeira fria traçada por $870 \mu \mathrm{m}$ indica a presença de protoestrelas associadas aos clumps densos. De fato, os YSOs Classe I candidatos a estar associados a N10 apresentados na Tabela 4.4 têm idades menores que a escala de tempo de fragmentação, sugerindo a possibilidade de formação de estrela desencadeada em condensações pré-existentes comprimidas pela pressão do gás ionizado, como o mecanismo "Radiation-Driven Implosion" propõe.

\subsubsection{A bolha N11}

As imagens no infravermelho da Figura 2.1 mostram N11, uma bolha que parece estar fisicamente conectada a N10 e estende-se por cerca de 3 pc na direção superior à direita. Inversamente, a distribuição molecular de N11 não sugere nenhuma ligação física com N10, uma vez que a emissão de ${ }^{13} \mathrm{CO}(1-0)$ entre 47 e $53 \mathrm{~km} \mathrm{~s}^{-1}$ não coincide com a emissão em $8.0 \mu \mathrm{m}$ de N11.

YSOs Classe I não aparecem sobre a casca da bolha N11 e poucos YSOs Classe II podem ser encontrados na direção de N11, como podemos ver na Figura 4.12. Entretanto, há uma notável concentração Discos de Transição em torno da parte superior da casca de N11. Especulamos que esse objeto seja um remanescente de uma região HII, na qual a falta de emissão em $20 \mathrm{~cm}$ nos leva a considerar que não há mais gás ionizado ali dentro. É provável que outra fonte de energia tenha provocado a formação destes YSOs, como a explosão de uma supernova tipo II, por exemplo.

\subsubsection{Uma pequena bolha à direita de N10}

A pequena bolha MWP1G013134+000580, de coordenadas centrais $l=13.134^{\circ}$ e $b=0.058^{\circ}$, tem dimensão menos que 2 pc. Interessantemente, essa pequena bolha deve ter a mesma distância de N10, visto que sua emissão em CO está contida no mesmo pico de velocidade principal, claramente observado nos mapas de canal com velocidades entre 51 e $53 \mathrm{~km} \mathrm{~s}^{-1}$ nas Figuras 4.2 e 4.3 .

Encontramos três objetos Classe I na região de MWP1G013134+000580. A idade da pequena bolha parece ser da mesma ordem de N10, conforme podemos inferir do estágio evolutivo dos YSOs encontrados nessa região. 


\subsection{Traçadores moleculares de formação estelar}

Enquanto CO traça o gás em larga escala na região, outras moléculas são indicadoras de formação estelar e nos permitem investigar a evolução química na região. Nessa seção discutiremos a caracterização de diferentes fases do gás na bolha N10.

\subsubsection{Ionização}

Íons moleculares normalmente são observados em regiões pré/protoestelares densas (e.g. Caselli et al., 2002). $\mathrm{HCO}^{+}$(íon formil) tem sido detectada na direção de outflows e em alguns casos, como mostra a Figura 4.19, apresenta uma morfologia de "borboleta", sugerindo excitação em cavidades produzidas por outflows (Podio et al., 2014, e suas referências). A emissão dessa espécie tem sido observada na direção de regiões de formação estelar (e.g. Goicoechea et al., 2012).

Savage \& Ziurys (2004) sugerem que, em nuvens escuras, as espécies reativas $H_{3}^{+}$proporcionam a reação química íon-neutro, e a maior parte da produção do íon formil é devida à reação:

$$
\mathrm{H}_{3}^{+}+\mathrm{CO} \rightarrow \mathrm{HCO}^{+}+\mathrm{H}_{2}
$$

De acordo com Graciá-Carpio et al. (2006), espera-se que a emissão dessa molécula provenha de gás molecular denso, em que a densidade seja $\geq 10^{4} \mathrm{~cm}^{-3}$. Assim, a distribuição de $\mathrm{HCO}^{+}$ em N10 caracteriza o gás molecular mais diretamente ligado à formação estelar.

O perfil de linha no pico de emissão de $\mathrm{HCO}^{+}(1-0)$ mostra asas com desvio tanto para o vermelho quanto para o azul, o que implica a presença de gás de alta velocidade na região. Essa componente em alta velocidade é traçada pela interação entre a região HII e o clump de alta densidade, o que é confirmado pela Figura 4.24.

\subsubsection{PDR}

Espera-se que as estrelas tipo O no interior da bolha N10 criem PDRs. O HCN (cianeto de nitrogênio) traça as camadas do gás denso que está sob a influência da radiação UV na região (Fuente et al., 1993).

$\mathrm{HCN}$ forma-se eficientemente nessa região, onde $\mathrm{C}^{+}$e $\mathrm{H}_{2}$ coexistem e podem reagir; ao reagir com $\mathrm{H}_{2}, \mathrm{C}^{+}$forma $\mathrm{CH}^{+}$. Nitrogênio atômico forma similarmente $\mathrm{NH}$, que retorna ao $\mathrm{C}^{+}$para, 
eventualmente, formar $\mathrm{HCN}$. Os radicais $\mathrm{CN}$ e $\mathrm{HCN}$ são conhecidos por serem especialmente abundantes em PDRs (Fuente et al., 1993).

O HCN normalmente é termalizado nos núcleos densos de nuvens moleculares, sendo portanto um bom traçador de gás molecular denso que está ativamente relacionado com o processo de formação estelar (Narayanan et al., 2008). Na região da bolha N10, a emissão de HCN (1 - 0) é intensa e indica a presença de PDRs ligadas à interação do gás com a fonte central. É esperado que tanto o $\mathrm{HCN}$ quanto o $\mathrm{HCO}^{+}$trace regiões densas associadas ao $\mathrm{CO}$, mas $\mathrm{HCN}$ traça predominantemente a região menos ionizada, se comparada com $\mathrm{HCO}^{+}$.

\subsubsection{Choques/Outflows}

A observação de linhas de SiO é uma poderosa ferramenta de diagnóstico para estudar outflows em regiões de formação estelar (e.g. Lefloch et al., 1998; Nisini et al., 2007; Gusdorf et al., 2008). A emissão de $\mathrm{SiO}$ na fase gasosa tem sido observada em estruturas associadas a jatos de YSOs embebidos em cores de baixa massa (Bally et al., 1993) e em regiões de formação de estrelas de alta massa (Acord et al., 1996).

A molécula SiO é produzida por meio de choques tipo-C (Schilke et al., 1997). Esse tipo de choque ocorre por meio de íons inseridos em um meio predominantemente neutro, ou seja, em nuvens moleculares com baixo grau de ionização e alta densidade (Draine et al., 1983). Choques tipo-C são assim chamados por ocorrerem de forma contínua (C de "continuous"), em comparação aos choques tipo-J, no qual a frente de choque é muito mais fina do que a camada de relaxamento pós-choque (J de "jump”, veja Hollenbach \& McKee, 1979, para maiores detalhes).

SiO é então produzida pela pulverização de silício por colisões grão-grão, seguida por reações com $\mathrm{O}$ e $\mathrm{O}_{2}$ também na fase gasosa. De acordo com Rizzo et al. (2005), SiO faz parte de um grupo de moléculas menos expostas ao campo UV, chamadas portanto de low $U V$, ou $L U V$, presentes principalmente nas bordas da PDR. A interação de fótons UV com os grãos modifica a química do gás molecular e pode evideciar a PDR avançando na nuvem molecular. SiO é, portanto, traçadora de condições de alta densidade e de choques de alta velocidade.

A presença de emissão $\mathrm{SiO}$ e as asas alargadas em $\mathrm{HCO}+$ é uma evidência de atividade de outflow na região (e.g. Vasyunina et al., 2011). Identificamos emissão SiO na direção de N10, concentrada em um pico principal que coincide com o clump de maior densidade na região. N10 apresenta, portanto, evidências de gás que sofreu choque possivelmente associado com outflows energéticos jovens. 


\subsubsection{Cores pré-estelares}

Cores pré-estelares apresentam uma composição química rica e inomogênea. Enquanto espécies como o $\mathrm{CO}$ sofrem depleção nesses núcleos densos, o $\mathrm{N}_{2} \mathrm{H}^{+}$(íon diazenílio) é um dos poucos íons moleculares que sobrevivem por bastante tempo nessa fase do gás.

Essa molécula é considerada um traçador molecular padrão de ambientes pré-estelares frios e quiescentes (e.g. Tafalla et al., 2006; Fontani et al., 2015). De acordo com Podio et al. (2014), o íon $\mathrm{N}_{2} \mathrm{H}^{+}$é formado através da reação íon-molécula:

$$
H_{3}^{+}+N_{2} \rightarrow N_{2} H^{+}+H_{2}
$$

sendo $H_{3}^{+}$produzido pela reação entre $\mathrm{H}_{2}$ e $\mathrm{H}_{2}^{+} \cdot \mathrm{H}_{2}^{+}$, por sua vez, é produzido pela ionização de $\mathrm{H}_{2}$ por raios cósmicos e destruído por recombinação dissociativa com elétrons (Podio et al. 2014). $\mathrm{N}_{2} \mathrm{H}^{+}$é, portanto, traçador de $\mathrm{H}_{2}$. Em YSOs Classe I, o íon $\mathrm{N}_{2} \mathrm{H}^{+}$pode ser destruído pelo outflow ou pela radiação UV proveniente da estrela que já se desligou de sua nuvem de origem (Alonso-Albi et al., 2010).

A transição $J=1-0$ de $\mathrm{N}_{2} \mathrm{H}^{+}$é particularmente interessante por geralmente apresentar baixa profundidade óptica em suas estruturas hiperfinas e possibilitar a medida das estruturas em cores de formação estelar densos (Caselli et al., 1995). De acordo com Codella et al. (2013), $\mathrm{N}_{2} \mathrm{H}^{+}$é um registro fóssil do gás pré-choque.

Enquanto moléculas com cadeia de carbono são abundantes nos estágios iniciais de evolução química e sofrem mais o efeito de depleção, a molécula $\mathrm{N}_{2} \mathrm{H}^{+}$é mais abundante nos estágios finais. Quando o $\mathrm{CO}$ se esgota na região, $\mathrm{N}_{2} \mathrm{H}^{+}$torna-se abundante nas partes mais densas (Sakai et al., 2008). Nesse reação:

$$
\mathrm{N}_{2} \mathrm{H}^{+}+\mathrm{CO} \rightarrow \mathrm{HCO}^{+}+\mathrm{N}_{2}
$$

Nós detectamos emissão intensa de $\mathrm{N}_{2} \mathrm{H}^{+}$em N10, caracterizando portanto a presença de cores pré-estelares na região de N10.

\subsubsection{Gás denso}

O CS é um traçador tradicional de gás denso (e.g. Snell et al., 1984; Bronfman et al., 1996; Helmich \& van Dishoeck, 1997). Embora tenhamos identificado a distribuição do gás molecular 
por meio da emissão de CO na direção da bolha N10 $\left(n_{H} \sim 5000 \mathrm{~cm}^{-3}\right)$, é esperado que as linhas de CS tracem regiões $>100$ vezes mais densas do que aquelas traçadas pela transição CO $(1-0)$ (e.g Bally et al., 1987; Yang et al., 1997). As moléculas que abrigam o enxofre potencialmente são um bom relógio para escalas de tempo relevantes na fase mais embebida de formação estelar (Li et al., 2015).

Além disso, estudos recentes realizados por Watson et al. (2016) mostram que a distribuição de CS nas bolhas infravermelhas indicam evidências de interações significativas entre objetos estelares jovens e o ambiente ao redor. A emissão de CS (3 - 2) em N10 indica, portanto, a presença de camadas de gás de alta densidade na nuvem molecular.

\subsubsection{Levantamento de linhas espectrais}

A Figura 4.26 mostra uma visão geral do espectro observado na direção da condensação mais densa em N10, em um intervalo de 86 a $100 \mathrm{GHz}$, aproximadamente. Identificamos 41 espécies moleculares, descritas na Tabela 4.7, e as classificamos de acordo com suas propriedades.

O resultado mais interessante desse levantamento foi a detecção de Moléculas Orgânicas Complexas (COMs, do inglês Complex Organic Molecules). COMs têm sido detectadas na direção de diversos ambientes astrofísicos como um produto da química entre o gás e os grãos de poeira. Na literatura astroquímica, as COMs são definidas como moléculas orgânicas que contém ao menos seis átomos pesados (Herbst \& van Dishoeck, 2009) e tem sido detectadas em regiões associadas com formação estelar (e.g. Blake et al., 1987; Cernicharo et al., 2012; Cazaux et al. 2003; Watanabe et al., 2015).

É esperado que a composição química de condensações nessas regiões reflita um processo sequencial, na qual moléculas simples sejam formadas na fase pré-colapso da nuvem fria, depois evaporem ao sofrer aquecimento por parte de uma estrela recém formada e formem COMs por meio de reações neutro-neutro e íon-neutro (Bottinelli et al., 2004).

Moléculas como CS e SO, assinaturas de outflow, foram detectadas. A presença de SiO indica choque na região. Esses resultados confirmam a atividade de formação estelar na condensação molecular observada. 


\section{Capítulo 6}

\section{Conclusões e Perspectivas}

"Us and them

And after all

We're only ordinary men"

Pink Floyd

Realizamos um estudo abrangente da bolha infravermelha N10 usando as linhas de emissão molecular ${ }^{12} \mathrm{CO}(J=1-0)$ and ${ }^{13} \mathrm{CO}(J=1-0)$, imagens infravermelhas Spitzer (levantamentos GLIMPSE e MIPSGAL), dados da emissão em 20 cm, observações APEX da emissão contínua em $870 \mu \mathrm{m}$ e fontes pontuais no infravermelho médio do catálogo WISE.

A fim de explorar a formação estelar em N10, estudamos transições dos traçadores moleculares $\mathrm{HCO}^{+}(1-0), \mathrm{HCN}(1-0), \mathrm{SiO}(2-1), \mathrm{N}_{2} \mathrm{H}^{+}(1-0)$ e $\mathrm{CS}(3-2)$ por meio de observações com o telescópio IRAM 30-m.

A seguir, descreveremos as principais conclusões a partir dos resultados obtidos.

Observamos a transição $J=1-0$ das espécies ${ }^{12} \mathrm{CO}$ e ${ }^{13} \mathrm{CO}$ no telescópio PMO $13.7 \mathrm{~m}$. A distribuição da emissão de CO mostrou que o gás ao redor da bolha N10 tem velocidade $V_{L S R}=52.6 \mathrm{~km} \mathrm{~s}^{-1}$, da qual pudemos estimar uma distância $D=4.7 \mathrm{kpc}$. Essas observações revelaram dois clumps de ${ }^{13} \mathrm{CO}$, com $M_{\text {LTE }} \sim 2 \times 10^{3} \mathrm{M}_{\odot}, M_{\text {virial }} \sim 8.5 \times 10^{3} \mathrm{M}_{\odot}$ e $M_{\text {Jeans }} \sim$ $6 \times 10^{3} \mathrm{M}_{\odot}$. Não é possível afirmar sobre a estabilidade gravitacional dos clumps, uma vez que as hipóteses adotadas para o cálculo de massas são grosseiras e podem levar a valores sub ou superestimados.

A emissão no contínuo de rádio e a presença da emissão em $24 \mu \mathrm{m}$ sugerem que existe ao menos uma fonte ionizante dentro da bolha. Nós estimamos um fluxo total de $20 \mathrm{~cm} F_{20 \mathrm{~cm}}=1.17 \mathrm{Jy}$ e uma densidade eletrônica $n_{e} \sim 130 \mathrm{~cm}^{-3}$, com um fluxo de fótons no contínuo de Lyman 
$N_{L y}=1.86 \times 10^{49}$ fótons ionizantes $\mathrm{s}^{-1}$.

Dois clumps de poeira fria foram identificados nas imagens do levantamento LABOCA/APEX. Para o clump mais denso, a partir da emissão em $870 \mu \mathrm{m}$, estimamos massa total $M_{\text {tot }}=240 \mathrm{M}_{\odot}$, raio médio $R_{D}=0.37 \mathrm{pc}$, densidade colunar $N\left(H_{2}\right)=6.3 \times 10^{22} \mathrm{~cm}^{-2}$ e densidade volumétrica média $n\left(H_{2}\right)=3.7 \times 10^{4} \mathrm{~cm}^{-3}$. Tais características físicas indicam que essa condensação é uma boa candidata a protoaglomerado.

Identificamos 234 YSOs na região como um todo, sendo 12 deles classificados como Classe I, 91 Classe II e 131 Discos de Transição. Ajustamos a SED para 9 dos YSOs Classe I identificados, os quais consideramos candidatos a pertencerem fisicamente à estrutura molecular de N10, e calculamos seus parâmetros físicos. A partir de modelos, encontramos idades estelares que vão desde $\sim 10^{3}$ até $10^{6}$ anos. Comparando a idade dinâmica estimada $\left(t_{d y n} \sim 9.17 \times 10^{4}\right.$ anos $) \mathrm{e}$ a escala de tempo de fragmentação $\left(t_{\text {frag }} \sim 1.5 \times 10^{6}\right.$ anos $)$, concluímos que a formação estelar pode ter sido desencadeada como consequência do mecanismo "Radiation-Driven Implosion", no qual a radiação ionizante comprime condensações pré-existentes.

Na imagem Spitzer em $8 \mu \mathrm{m}$, a bolha infravermelha N11 pode ser vista na direção de N10, entretanto não há indícios de que elas estejam conectadas fisicamente uma com a outra. Objetos Classe II aparecem na direção de N11, sugerindo que esta pode ser uma remanescente de região HII. Uma terceira bolha infravermelha, a pequena MWP1G013134+000580, aparece no campo observado pelo PMO 13.7-m e, interessantemente, apresenta emissão de CO na mesma velocidade da emissão principal de N10 e parece para abrigar alguns YSOs evoluídos.

Através das observações realizadas com o telescópio IRAM 30-m, obtivemos os mapas de emissão integrada da transição das espécies moleculares: $\mathrm{HCO}^{+}(1-0), \mathrm{HCN}(1-0), \mathrm{SiO}(2-1)$, $\mathrm{N}_{2} \mathrm{H}^{+}(1-0)$ e CS $(3-2)$. Dessa forma, identificamos diferentes fases do gás associado a N10.

A região de ionização foi caracterizada pela emissão de $\mathrm{HCO}^{+}$. A distribuição espacial é característica de excitação provocada por outflows e evidencia o gás molecular relacionado a formação estelar. As camadas de gás denso presentes nas PDRs foram reveladas pela emissão de HCN. A região de outflows foi traçada pela emissão de $\mathrm{SiO}$, molécula produzida por choques tipo-C. A presença de cores pré-estelares foi confirmada pela detecção do íon $\mathrm{N}_{2} \mathrm{H}^{+}$na nuvem molecular, e a detecção de CS indica gás denso em N10.

Interessantemente, a distribuição espacial dessas moléculas não abrange as bolhas N11 e MWP1G013134+000580. Isso indica que apenas N10 apresenta características de região de formação estelar. 
Um levantamento de linhas espectrais na direção da condensação mais densa de N10 nos permitiu identificar 41 moléculas na banda de 3-mm do telescópio IRAM 30-m. A presença de COMs e moléculas que abrigam enxofre indica atividade de outflows e formação estelar.

\subsection{Perspectivas}

Tendo em vista o sucesso de nossas observações no telescópio IRAM 30-m, os estudos aqui apresentados podem ser aprofundados. Os mapas de intensidade integrada pode nos fornecer informações acerca das condensações e podemos explorar suas propriedades físicas como dimensão, temperatura e massa. Futuramente, podemos comparar os resultados obtidos com outras bolhas infravermelhas.

O estudo das linhas identificadas na integração profunda necessitam de maior análise, da qual podemos:

- Caracterizar os parâmetros das linhas observadas no levantamento espectral;

- Estudar a abundância relativa das espécies;

- Comparar abundâncias observadas com modelos químicos;

- Investigar o estágio evolutivo dos clumps utilizando a razão $\mathrm{CO} / \mathrm{N} 2 \mathrm{H}^{+}$, por exemplo. 


\section{Referências Bibliográficas}

Acord, J. M., Churchwell, E., \& Walmsley, C. M. 1996, Bulletin of the American Astronomical Society, $28,50.01$

Alonso-Albi, T., Fuente, A., Crimier, N., et al. 2010, A\&A, 518, A52

Amôres, E. B., \& Lépine, J. R. D. 2005, AJ, 130, 659

Anderson, L. D., Bania, T. M., Balser, D. S., et al. 2014, ApJS, 212, 1

Anderson, L. D., Deharveng, L., Zavagno, A., et al. 2015, ApJ, 800, 101

Bally, J., Stark, A. A., Wilson, R. W., \& Henkel, C. 1987, ApJS, 65, 13

Bally, J., Lada, E. A., \& Lane, A. P. 1993, ApJ, 418, 322

Beaumont, C. N., \& Williams, J. P. 2010, ApJ, 709, 791

Benjamin, R. A., Churchwell, E., Babler, B. L., et al. 2003, PASP, 115, 953

Bergin, E. A., \& Tafalla, M. 2007, ARA\&A, 45, 339

Beuther, H., Linz, H., Henning, T., et al. 2011, A\&A, 531, AA26

Blake, G. A., Sutton, E. C., Masson, C. R., \& Phillips, T. G. 1987, ApJ, 315, 621

Bottinelli, S., Ceccarelli, C., Lefloch, B., et al. 2004, ApJ, 615, 354

Brand, J., \& Blitz, L. 1993, A\&A, 275, 67

Bronfman, L., Nyman, L.-A., \& May, J. 1996, A\&AS, 115, 81

Cappa, C. E., Duronea, N., Firpo, V., et al. 2016, A\&A, 585, A30 
Caselli, P., Myers, P. C., \& Thaddeus, P. 1995, ApJ, 455, L77

Caselli, P., Benson, P. J., Myers, P. C., \& Tafalla, M. 2002, ApJ, 572, 238

Cazaux, S., Tielens, A. G. G. M., Ceccarelli, C., et al. 2003, ApJ, 593, L51

Cernicharo, J., Marcelino, N., Roueff, E., et al. 2012, ApJ, 759, L43

Charnley, S. B., Tielens, A. G. G. M., \& Millar, T. J. 1992, ApJ, 399, L71

Chiar, J. E., Tielens, A. G. G. M., Whittet, D. C. B., et al. 2000, ApJ, 537, 749

Churchwell, E., Povich, M. S., Allen, D., et al. 2006, ApJ, 649, 759

Churchwell, E., Watson, D. F., Povich, M. S., et al. 2007, ApJ, 670, 428

Codella, C., Viti, S., Ceccarelli, C., et al. 2013, ApJ, 776, 52

Condon, J. J., Cotton, W. D., Greisen, E. W., et al. 1998, AJ, 115, 1693

Cutri, R. M., \& IPAC/WISE Science Data Center Team 2011, Bulletin of the American Astronomical Society, 43, 301.02

Cutri, R. M., et al. 2013, VizieR Online Data Catalog, 2328, 0

Deharveng, L., Lefloch, B., Zavagno, A., et al. 2003, A\&A, 408, L25

Deharveng, L., Lefloch, B., Kurtz, S., et al. 2008, A\&A, 482, 585

Deharveng, L., Schuller, F., Anderson, L. D., et al. 2010, A\&A, 523, A6

Dewangan, L. K., Ojha, D. K., Anandarao, B. G., Ghosh, S. K., \& Chakraborti, S. 2012, ApJ, 756,151

Deharveng, L., Zavagno, A., Samal, M. R., et al. 2015, A\&A, 582, A1

Dewangan, L. K., Ojha, D. K., Grave, J. M. C., \& Mallick, K. K. 2015, MNRAS, 446, 2640

Dobashi, K., Yonekura, Y., Matsumoto, T., et al. 2002, EAS Publications Series, 4, 139

Draine, B. T., Roberge, W. G., \& Dalgarno, A. 1983, ApJ, 264, 485

Duronea, N. U., Vasquez, J., Gómez, L., et al. 2015, A\&A, 582, A2 
Elmegreen, B. G., \& Lada, C. J. 1977, ApJ, 214, 725

Fazio, G. G., Hora, J. L., Allen, L. E., et al. 2004, ApJS, 154, 10

Feigelson, E. D., \& Montmerle, T. 1999, ARA\&A, 37, 363

Fontani, F., Caselli, P., Palau, A., Bizzocchi, L., \& Ceccarelli, C. 2015, ApJ, 808, L46

Fuente, A., Martin-Pintado, J., Cernicharo, J., \& Bachiller, R. 1993, A\&A, 276, 473

Gama, D.R.G. 2012, Dissertação de Mestrado, Formação estelar no complexo de nuvens moleculares em Monoceros. Dissertação de Mestrado, Instituto de Astronomia, Geofísica e Ciências Atmosféricas, Universidade de São Paulo, São Paulo. Disponível em: http://www.teses.usp.br/teses/disponiveis/14/14131/tde-29062012-100922/

Gama, D.R.G., Lepine, J.R.D., Wu, Y., Yuan, J., \& Mendoza, E. 2016, Manuscript submitted to ApJ for publication

Garden, R. P., Hayashi, M., Hasegawa, T., Gatley, I., \& Kaifu, N. 1991, ApJ, 374, 540

Goicoechea, J. R., Cernicharo, J., Karska, A., et al. 2012, A\&A, 548, A77

Graciá-Carpio, J., García-Burillo, S., Planesas, P., \& Colina, L. 2006, ApJ, 640, L135

Gusdorf, A., Pineau Des Forêts, G., Cabrit, S., \& Flower, D. R. 2008, A\&A, 490, 695

Gutermuth, R. A., Megeath, S. T., Myers, P. C., et al. 2009, ApJS, 184, 18

Helfand, D. J., Becker, R. H., White, R. L., Fallon, A., \& Tuttle, S. 2006, AJ, 131, 2525

Helmich, F. P., \& van Dishoeck, E. F. 1997, A\&AS, 124,

Herbst, E., \& van Dishoeck, E. F. 2009, ARA\&A, 47, 427

Hollenbach, D., \& McKee, C. F. 1979, ApJS, 41, 555

Hollenbach, D. J., \& Tielens, A. G. G. M. 1999, Reviews of Modern Physics, 71, 173

Hou, L. G., \& Han, J. L. 2014, A\&A, 569, AA125

Huang, S.-S. 1954, AJ, 59, 137

Jeans, J. H. 1928, Cambridge [Eng.] The University press, 1928., 
Kendrew, S., Simpson, R., Bressert, E., et al. 2012, ApJ, 755, 71

Kerr, F. J., \& Knapp, G. R. 1974, Galactic Radio Astronomy, 60, 179

Koenig, X. P., Leisawitz, D. T., Benford, D. J., et al. 2012, ApJ, 744, 130

Koenig, X. P., \& Leisawitz, D. T. 2014, ApJ, 791, 131

Kwok, S. 2007, Physics and Chemistry of the Interstellar Medium by Sun Kwok. University Science Books, 2007.,

Lee, J.-K., Walsh, A. J., Burton, M. G., \& Ashley, M. C. B. 2001, MNRAS, 324, 1102

Lefloch, B., \& Lazareff, B. 1994, A\&A, 289, 559

Lefloch, B., Lazareff, B., \& Castets, A. 1997, A\&A, 324, 249

Lefloch, B., Castets, A., Cernicharo, J., \& Loinard, L. 1998, ApJ, 504, L109

Lefloch, B., Cernicharo, J., Cabrit, S., \& Cesarsky, D. 2005, A\&A, 433, 217

Li, J., Wang, J., Zhu, Q., Zhang, J., \& Li, D. 2015, ApJ, 802, 40

Liu, T., Wu, Y., Zhang, H., \& Qin, S.-L. 2012, ApJ, 751, 68

Liu, H.-L., Wu, Y., Li, J., et al. 2015, ApJ, 798, 30

Liu, H.-L., Li, J.-Z., Wu, Y., et al. 2016, ApJ, 818, 95

Lockman, F. J. 1989, ApJS, 71, 469

Ma, Y., Zhou, J., Esimbek, J., et al. 2013, Ap\&SS, 345, 297

Hollenbach, D. J., \& Tielens, A. G. G. M. 1996, ApJ, 466, 561

Matsakis, D. N., Evans, N. J., II, Sato, T., \& Zuckerman, B. 1976, AJ, 81, 172

McKee, C. F., \& Ostriker, E. C. 2007, ARA\&A, 45, 565

Minier, V., \& Booth, R. S. 2002, A\&A, 387, 179

Minier, V., Ellingsen, S. P., Norris, R. P., \& Booth, R. S. 2003, A\&A, 403, 1095

Miettinen, O. 2012, A\&A, 542, A101 
Motte, F., Schilke, P., \& Lis, D. C. 2003, ApJ, 582, 277

Narayanan, D., Cox, T. J., Shirley, Y., et al. 2008, ApJ, 684, 996-1008

Nieten, C., Neininger, N., Guélin, M., et al. 2006, A\&A, 453, 459

Nisini, B., Codella, C., Giannini, T., et al. 2007, A\&A, 462, 163

Panagia, N., \& Walmsley, C. M. 1978, A\&A, 70, 411

Paladini, R., Umana, G., Veneziani, M., et al. 2012, ApJ, 760, 149

Pandian, J. D., Momjian, E., \& Goldsmith, P. F. 2008, A\&A, 486, 191

Perault, M., Omont, A., Simon, G., et al. 1996, A\&A, 315, L165

Podio, L., Lefloch, B., Ceccarelli, C., Codella, C., \& Bachiller, R. 2014, A\&A, 565, A64

Reich, W., Fuerst, E., Reich, P., \& Reif, K. 1990, A\&AS, 85, 633

Reipurth, B. 1983, A\&A, 117, 183

Rieke, G. H., Young, E. T., Engelbracht, C. W., et al. 2004, ApJS, 154, 25

Rizzo, J. R., Fuente, A., \& García-Burillo, S. 2005, ApJ, 634, 1133

Robitaille, T. P., Whitney, B. A., Indebetouw, R., \& Wood, K. 2007, ApJS, 169, 328

Roman-Duval, J., Jackson, J. M., Heyer, M., et al. 2009, ApJ, 699, 1153

Sakai, T., Sakai, N., Kamegai, K., et al. 2008, ApJ, 678, 1049-1069

Salpeter, E. E. 1955, ApJ, 121, 161

Samal, M. R., Zavagno, A., Deharveng, L., et al. 2014, A\&A, 566, A122

Sartori, M. J., Lépine, J. R. D., \& Dias, W. S. 2003, A\&A, 404, 913

Sault, R. J., Teuben, P. J., \& Wright, M. C. H. 1995, Astronomical Data Analysis Software and Systems IV, 77, 433

Savage, C., \& Ziurys, L. M. 2004, ApJ, 616, 966 
Shan, W., Yang, J., Shi, S., et al. 2012, Transactions on Terahertz Science and Technology, 2, 593

Schilke, P., Walmsley, C. M., Pineau des Forets, G., \& Flower, D. R. 1997, A\&A, 321, 293

Schinnerer, E., Meidt, S. E., Pety, J., et al. 2013, ApJ, 779, 42

Schuller, F., Menten, K. M., Contreras, Y., et al. 2009, A\&A, 504, 415

Shu, F. H. 1977, ApJ, 214, 488

Shu, F. H., Adams, F. C., \& Lizano, S. 1987, ARA\&A, 25, 23

Simpson, R. J., Povich, M. S., Kendrew, S., et al. 2012, VizieR Online Data Catalog, 742, 42442

Snell, R. L., Mundy, L. G., Goldsmith, P. F., Evans, N. J., II, \& Erickson, N. R. 1984, ApJ, 276, 625

Stahler, S. W., \& Palla, F. 2005, The Formation of Stars, by Steven W. Stahler, Francesco Palla, pp. 865. ISBN 3-527-40559-3. Wiley-VCH , January 2005

Stahler, S. W., \& Yen, J. J. 2010, MNRAS, 407, 2434

Strom, K. M., Strom, S. E., Edwards, S., Cabrit, S., \& Skrutskie, M. F. 1989, AJ, 97, 1451

Szymczak, M., Hrynek, G., \& Kus, A. J. 2000, A\&AS, 143, 269

Tackenberg, J., Beuther, H., Henning, T., et al. 2012, A\&A, 540, AA113

Tafalla, M., Santiago-García, J., Myers, P. C., et al. 2006, A\&A, 455, 577

Thompson, M. A., Urquhart, J. S., Moore, T. J. T., \& Morgan, L. K. 2012, MNRAS, 421, 408

Tielens, A. G. G. M., \& Hagen, W. 1982, A\&A, 114, 245

Ungerechts, H., Umbanhowar, P., \& Thaddeus, P. 2000, ApJ, 537, 221

Vasyunina, T., Linz, H., Henning, T., et al. 2011, A\&A, 527, A88

Watanabe, Y., Sakai, N., López-Sepulcre, A., et al. 2015, ApJ, 809, 162

Watson, C., Povich, M. S., Churchwell, E. B., et al. 2008, ApJ, 681, 1341 
Watson, C., Devine, K., Quintanar, N., \& Candelaria, T. 2016, ApJ, 818, 71

Wienen, M., Wyrowski, F., Schuller, F., et al. 2012, A\&A, 544, A146

Wilking, B. A. 1989, PASP, 101, 229

Wilson, T. L., \& Rood, R. 1994, ARA\&A, 32, 191

Whitworth, A. P., Bhattal, A. S., Chapman, S. J., Disney, M. J., \& Turner, J. A. 1994, MNRAS, 268,291

Whitworth, A. P., \& Francis, N. 2002, MNRAS, 329, 641

Wright, E. L., Eisenhardt, P. R. M., Mainzer, A. K., et al. 2010, AJ, 140, 1868

Yam, O., Paglione, T. A. D., Jackson, J. M., \& Bolatto, A. D. 1998, IX Latin American Regional IAU Meeting, "Focal Points in Latin American Astronomy",

Yang, J., Ohashi, N., Yan, J., et al. 1997, ApJ, 475, 683

Yuan, J.-H., Wu, Y., Li, J. Z., \& Liu, H. 2014, ApJ, 797, 40

Zavagno, A., Anderson, L. D., Russeil, D., et al. 2010, A\&A, 518, L101

Zinnecker, H., \& Yorke, H. W. 2007, ARA\&A, 45, 481 
Apêndice 



\section{Lista de YSOs identificados em N10}

Tabela A.1 - Candidatos a objetos estelares jovens (YSOs) na direção da região N10.

\begin{tabular}{|c|c|c|c|c|c|c|c|c|}
\hline \multirow{2}{*}{$\begin{array}{c}\text { Candidata } \\
\#\end{array}$} & \multirow{2}{*}{$\begin{array}{c}1 \\
{\left[{ }^{\circ}\right]}\end{array}$} & \multirow{2}{*}{$\begin{array}{c}\mathrm{b} \\
{\left[{ }^{\circ}\right]}\end{array}$} & \multirow{2}{*}{$\begin{array}{c}\text { Identificação } \\
\text { WISE }\end{array}$} & \multicolumn{4}{|c|}{ Fluxos (mag) } & \multirow[t]{2}{*}{ Classificação } \\
\hline & & & & [3.4 $\mu \mathrm{m}]$ & {$[4.6 \mu \mathrm{m}]$} & {$[12 \mu \mathrm{m}]$} & {$[22 \mu \mathrm{m}]$} & \\
\hline 1 & 13.1141 & 0.0566 & J181354.14-173204.6 & 12.651 & 11.220 & 7.212 & 4.212 & Classe I \\
\hline 2 & 13.1385 & 0.0593 & $\mathrm{~J} 181356.48-173043.0$ & 10.428 & 9.181 & 5.204 & 1.246 & Classe I \\
\hline 3 & 13.1383 & 0.0566 & $\mathrm{~J} 181357.08-173048.2$ & 11.451 & 9.848 & 5.595 & 1.771 & Classe I \\
\hline 4 & 13.2124 & 0.0402 & J181409.60-172722.1 & 9.274 & 7.294 & 4.049 & 1.312 & Classe I \\
\hline 5 & 13.2007 & 0.0286 & J181410.77-172819.3 & 9.995 & 8.855 & 6.081 & 0.734 & Classe I \\
\hline 6 & 13.2126 & 0.0477 & J181407.98-172708.4 & 9.978 & 7.224 & 3.903 & 1.187 & Classe I \\
\hline 7 & 13.1818 & 0.0608 & $\mathrm{~J} 181401.37-172823.3$ & 11.937 & 8.766 & 6.442 & 0.629 & Classe I \\
\hline 8 & 13.2015 & 0.0554 & J181404.94-172730.5 & 10.081 & 8.552 & 4.069 & -0.537 & Classe I \\
\hline 9 & 13.2016 & 0.0681 & $\mathrm{~J} 181402.15-172708.2$ & 10.877 & 10.015 & 6.538 & 2.244 & Classe I \\
\hline 10 & 13.2155 & -0.0350 & J181426.60-172921.9 & 9.291 & 7.367 & 4.237 & 1.531 & Classe I \\
\hline 11 & 13.2396 & -0.0057 & $\mathrm{~J} 181423.03-172715.2$ & 12.263 & 11.050 & 8.256 & 5.221 & Classe I \\
\hline 12 & 13.1931 & 0.0888 & J181356.55-172659.5 & 12.308 & 10.741 & 6.260 & 3.168 & Classe I \\
\hline 13 & 13.1114 & 0.1134 & $\mathrm{~J} 181341.27-173035.2$ & 10.397 & 9.934 & 8.657 & 4.825 & Classe II \\
\hline 14 & 13.1135 & -0.0134 & $\mathrm{~J} 181409.56-173407.1$ & 11.620 & 11.198 & 9.670 & 7.655 & Classe II \\
\hline 15 & 13.1389 & -0.0279 & J181415.81-173311.7 & 11.511 & 11.061 & 9.146 & 6.480 & Classe II \\
\hline 16 & 13.1384 & -0.0162 & J181413.16-173253.3 & 11.235 & 10.748 & 8.809 & 6.800 & Classe II \\
\hline 17 & 13.1147 & -0.0195 & J181411.06-173413.8 & 11.343 & 11.046 & 8.968 & 7.287 & Classe II \\
\hline 18 & 13.1449 & -0.0193 & J181414.65-173237.9 & 11.031 & 10.757 & 8.852 & 6.340 & Classe II \\
\hline 19 & 13.1527 & -0.0295 & $\mathrm{~J} 181417.83-173231.1$ & 10.529 & 10.021 & 7.684 & 5.341 & Classe II \\
\hline 20 & 13.1543 & -0.0234 & J181416.66-173215.4 & 10.758 & 10.158 & 8.237 & 6.232 & Classe II \\
\hline 21 & 13.1647 & -0.0310 & J181419.59-173155.6 & 11.511 & 10.498 & 9.022 & 6.565 & Classe II \\
\hline 22 & 13.1577 & -0.0263 & J181417.72-173209.5 & 10.568 & 10.225 & 8.441 & 5.908 & Classe II \\
\hline 23 & 13.1919 & -0.0358 & J181423.94-173037.7 & 10.433 & 10.010 & 8.891 & 6.481 & Classe II \\
\hline 24 & 13.1704 & -0.0343 & $\mathrm{~J} 181421.01-173143.2$ & 10.077 & 9.621 & 8.265 & 5.976 & Classe II \\
\hline 25 & 13.2038 & -0.0395 & $\mathrm{~J} 181426.20-173006.5$ & 8.941 & 8.625 & 7.104 & 5.237 & Classe II \\
\hline 26 & 13.1103 & 0.0155 & $\mathrm{~J} 181402.78-173327.7$ & 10.560 & 10.300 & 8.813 & 6.951 & Classe II \\
\hline 27 & 13.1370 & -0.0034 & $\mathrm{~J} 181410.17-173235.5$ & 9.904 & 9.313 & 8.291 & 5.669 & Classe II \\
\hline 28 & 13.1452 & -0.0016 & $\mathrm{~J} 181410.76-173206.7$ & 10.542 & 10.097 & 7.285 & 4.779 & Classe II \\
\hline 29 & 13.1257 & -0.0028 & J181408.67-173310.3 & 11.147 & 10.754 & 8.995 & 6.637 & Classe II \\
\hline 30 & 13.1362 & -0.0003 & J181409.38-173232.9 & 11.275 & 10.635 & 8.559 & 5.484 & Classe II \\
\hline
\end{tabular}


Tabela A.1 - continua

\begin{tabular}{|c|c|c|c|c|c|c|c|c|}
\hline \multirow{2}{*}{$\begin{array}{c}\text { Candidata } \\
\# \\
\end{array}$} & \multirow{2}{*}{$\begin{array}{c}1 \\
{\left[{ }^{\circ}\right]}\end{array}$} & \multirow{2}{*}{$\begin{array}{l}\mathrm{b} \\
{\left[{ }^{\circ}\right]}\end{array}$} & \multirow{2}{*}{$\begin{array}{c}\text { Identificação } \\
\text { WISE }\end{array}$} & \multicolumn{4}{|c|}{ Fluxos (mag) } & \multirow[t]{2}{*}{ Classificação } \\
\hline & & & & {$[3.4 \mu \mathrm{m}]$} & {$[4.6 \mu \mathrm{m}]$} & {$[12 \mu \mathrm{m}]$} & {$[22 \mu \mathrm{m}]$} & \\
\hline 31 & 13.1269 & 0.0325 & $\mathrm{~J} 181401.03-173205.8$ & 10.224 & 9.903 & 8.315 & 6.522 & Classe II \\
\hline 32 & 13.1505 & -0.0153 & J181414.43-173213.6 & 10.636 & 10.131 & 8.669 & 5.761 & Classe II \\
\hline 33 & 13.1611 & -0.0034 & J181413.08-173119.4 & 10.832 & 10.136 & 8.904 & 5.365 & Classe II \\
\hline 34 & 13.1541 & 0.0016 & J181411.12-173133.1 & 10.988 & 10.541 & 8.519 & 5.942 & Classe II \\
\hline 35 & 13.1907 & -0.0196 & $\mathrm{~J} 181420.20-173013.7$ & 10.467 & 9.685 & 8.212 & 7.706 & Classe II \\
\hline 36 & 13.1771 & -0.0256 & J181419.91-173106.9 & 10.485 & 10.058 & 8.892 & 7.684 & Classe II \\
\hline 37 & 13.1959 & -0.0330 & $\mathrm{~J} 181423.81-173020.5$ & 10.700 & 10.302 & 8.892 & 6.814 & Classe II \\
\hline 38 & 13.1979 & -0.0191 & J181420.96-172950.1 & 11.156 & 10.463 & 9.027 & 6.777 & Classe II \\
\hline 39 & 13.1797 & -0.0231 & J181419.68-173054.4 & 10.657 & 10.390 & 8.281 & 6.066 & Classe II \\
\hline 40 & 13.2025 & -0.0207 & J181421.88-172938.4 & 10.453 & 9.805 & 8.237 & 5.412 & Classe II \\
\hline 41 & 13.1850 & -0.0311 & $\mathrm{~J} 181422.08-173051.7$ & 11.027 & 10.458 & 8.722 & 6.238 & Classe II \\
\hline 42 & 13.1763 & -0.0096 & J181416.28-173041.9 & 9.523 & 8.906 & 7.872 & 4.966 & Classe II \\
\hline 43 & 13.1529 & 0.0133 & J181408.39-173116.8 & 11.277 & 10.554 & 9.114 & 5.257 & Classe II \\
\hline 44 & 13.1778 & 0.0060 & J181413.01-173010.3 & 9.489 & 8.862 & 7.858 & 3.911 & Classe II \\
\hline 45 & 13.1627 & 0.0214 & J181407.78-173031.9 & 9.323 & 8.688 & 5.991 & 2.136 & Classe II \\
\hline 46 & 13.1732 & 0.0086 & J181411.88-173020.6 & 10.801 & 10.366 & 7.612 & 3.212 & Classe II \\
\hline 47 & 13.1599 & 0.0238 & J181406.92-173036.4 & 9.450 & 8.858 & 6.121 & 2.438 & Classe II \\
\hline 48 & 13.1904 & 0.0215 & J181411.09-172903.8 & 11.423 & 10.865 & 9.270 & 2.260 & Classe II \\
\hline 49 & 13.1951 & 0.0128 & J181413.58-172903.9 & 9.707 & 8.801 & 6.974 & 2.114 & Classe II \\
\hline 50 & 13.2076 & 0.0148 & J181414.64-172821.2 & 9.698 & 9.104 & 6.214 & 1.898 & Classe II \\
\hline 51 & 13.1538 & 0.0598 & J181358.24-172953.7 & 9.997 & 9.382 & 8.095 & 4.876 & Classe II \\
\hline 52 & 13.1348 & 0.0557 & J181356.85-173101.0 & 9.628 & 8.946 & 5.814 & 2.094 & Classe II \\
\hline 53 & 13.1236 & 0.0950 & J181346.80-173028.4 & 10.652 & 10.249 & 8.394 & 5.963 & Classe II \\
\hline 54 & 13.1157 & 0.0930 & J181346.30-173057.0 & 11.448 & 11.137 & 9.105 & 7.158 & Classe II \\
\hline 55 & 13.1554 & 0.0962 & $\mathrm{~J} 181350.37-172845.8$ & 10.915 & 10.286 & 9.060 & 8.037 & Classe II \\
\hline 56 & 13.2163 & 0.0706 & J181403.36-172617.6 & 10.970 & 10.459 & 7.987 & 4.483 & Classe II \\
\hline 57 & 13.2151 & 0.0670 & $\mathrm{~J} 181404.00-172627.5$ & 11.001 & 10.383 & 7.583 & 5.756 & Classe II \\
\hline 58 & 13.2071 & -0.0271 & J181423.84-172934.7 & 9.319 & 8.922 & 7.300 & 4.959 & Classe II \\
\hline 59 & 13.2185 & -0.0141 & $\mathrm{~J} 181422.34-172836.2$ & 10.337 & 9.983 & 7.966 & 4.871 & Classe II \\
\hline 60 & 13.2078 & -0.0178 & J181421.88-172916.6 & 9.124 & 8.589 & 7.523 & 4.744 & Classe II \\
\hline 61 & 13.2179 & -0.0213 & $\mathrm{~J} 181423.85-172850.7$ & 10.761 & 10.112 & 7.705 & 5.931 & Classe II \\
\hline 62 & 13.2204 & 0.0050 & J181418.36-172757.4 & 10.030 & 9.534 & 8.327 & 4.625 & Classe II \\
\hline 63 & 13.2143 & 0.0017 & $\mathrm{~J} 181418.35-172822.5$ & 10.872 & 10.285 & 8.820 & 4.903 & Classe II \\
\hline 64 & 13.2536 & -0.0052 & J181424.60-172629.9 & 10.132 & 9.667 & 7.969 & 5.408 & Classe II \\
\hline 65 & 13.2532 & 0.0007 & $\mathrm{~J} 181423.25-172621.3$ & 11.055 & 10.557 & 8.166 & 5.727 & Classe II \\
\hline 66 & 13.2621 & 0.0030 & $\mathrm{~J} 181423.79-172549.1$ & 11.615 & 10.736 & 9.077 & 5.965 & Classe II \\
\hline 67 & 13.2553 & -0.0207 & $\mathrm{~J} 181428.22-172651.4$ & 9.615 & 9.146 & 7.810 & 5.657 & Classe II \\
\hline 68 & 13.2630 & -0.0075 & $\mathrm{~J} 181426.23-172604.1$ & 11.537 & 10.949 & 9.479 & 5.131 & Classe II \\
\hline 69 & 13.2440 & -0.0054 & $\mathrm{~J} 181423.50-172700.7$ & 10.056 & 9.031 & 7.095 & 4.135 & Classe II \\
\hline 70 & 13.2507 & -0.0041 & $\mathrm{~J} 181424.00-172637.2$ & 10.300 & 9.913 & 7.819 & 5.102 & Classe II \\
\hline 71 & 13.2489 & 0.0039 & $\mathrm{~J} 181422.01-172629.3$ & 7.988 & 7.294 & 5.927 & 4.050 & Classe II \\
\hline 72 & 13.2484 & -0.0065 & $\mathrm{~J} 181424.27-172648.7$ & 10.165 & 9.770 & 7.610 & 5.207 & Classe II \\
\hline 73 & 13.2669 & -0.0343 & $\mathrm{~J} 181432.62-172638.0$ & 10.417 & 9.654 & 8.019 & 7.414 & Classe II \\
\hline 74 & 13.2583 & -0.0373 & $\mathrm{~J} 181432.25-172710.3$ & 11.407 & 10.567 & 8.639 & 8.173 & Classe II \\
\hline
\end{tabular}


Tabela A.1 - continua

\begin{tabular}{|c|c|c|c|c|c|c|c|c|}
\hline \multirow{2}{*}{$\begin{array}{c}\text { Candidata } \\
\#\end{array}$} & \multirow{2}{*}{$\begin{array}{c}1 \\
{\left[{ }^{\circ}\right]}\end{array}$} & \multirow{2}{*}{$\begin{array}{c}\mathrm{b} \\
{\left[{ }^{\mathrm{o}}\right]} \\
\end{array}$} & \multirow{2}{*}{$\begin{array}{c}\text { Identificação } \\
\text { WISE }\end{array}$} & \multicolumn{4}{|c|}{ Fluxos (mag) } & \multirow[t]{2}{*}{ Classificação } \\
\hline & & & & {$[3.4 \mu \mathrm{m}]$} & {$[4.6 \mu \mathrm{m}]$} & {$[12 \mu \mathrm{m}]$} & {$[22 \mu \mathrm{m}]$} & \\
\hline 75 & 13.2301 & 0.0140 & J181417.54-172711.3 & 10.361 & 9.642 & 7.410 & 6.239 & Classe II \\
\hline 76 & 13.2255 & 0.0156 & J181416.61-172723.1 & 9.430 & 8.754 & 7.473 & 4.366 & Classe II \\
\hline 77 & 13.2451 & 0.0341 & J181414.88-172549.3 & 10.995 & 10.254 & 7.405 & 4.293 & Classe II \\
\hline 78 & 13.2404 & 0.0107 & J181419.49-172644.6 & 10.908 & 9.870 & 7.559 & 7.685 & Classe II \\
\hline 79 & 13.2343 & 0.0419 & J181411.87-172610.1 & 9.853 & 9.426 & 7.266 & 4.838 & Classe II \\
\hline 80 & 13.2499 & 0.0332 & J181415.66-172535.5 & 10.933 & 10.088 & 7.836 & 4.427 & Classe II \\
\hline 81 & 13.2636 & 0.0175 & J181420.79-172519.2 & 9.982 & 9.342 & 8.087 & 5.534 & Classe II \\
\hline 82 & 13.2574 & 0.0339 & J181416.42-172510.8 & 11.900 & 11.196 & 8.401 & 5.181 & Classe II \\
\hline 83 & 13.2392 & 0.0430 & J181412.20-172552.7 & 10.673 & 10.118 & 8.891 & 5.524 & Classe II \\
\hline 84 & 13.2558 & 0.0378 & J181415.36-172509.1 & 11.519 & 11.054 & 8.415 & 5.495 & Classe II \\
\hline 85 & 13.1292 & 0.1156 & J181342.93-172935.3 & 10.796 & 10.527 & 8.517 & 6.073 & Classe II \\
\hline 86 & 13.1461 & 0.1132 & J181345.50-172845.9 & 11.101 & 10.645 & 8.279 & 6.229 & Classe II \\
\hline 87 & 13.1350 & 0.1103 & J181344.81-172926.0 & 8.488 & 7.950 & 6.759 & 5.174 & Classe II \\
\hline 88 & 13.1616 & 0.1054 & J181349.09-172810.6 & 11.137 & 10.402 & 8.815 & 5.834 & Classe II \\
\hline 89 & 13.1883 & 0.0892 & J181355.88-172714.1 & 7.892 & 7.571 & 6.055 & 3.938 & Classe II \\
\hline 90 & 13.1815 & 0.1000 & J181352.66-172716.9 & 10.393 & 9.975 & 7.960 & 6.182 & Classe II \\
\hline 91 & 13.1945 & 0.1155 & J181350.82-172609.0 & 10.328 & 10.050 & 8.788 & 6.638 & Classe II \\
\hline 92 & 13.1833 & 0.1066 & J181351.44-172659.9 & 9.333 & 9.015 & 7.827 & 5.732 & Classe II \\
\hline 93 & 13.2189 & 0.0964 & J181357.99-172524.8 & 9.412 & 8.517 & 6.523 & 2.770 & Classe II \\
\hline 94 & 13.2282 & 0.0955 & J181359.30-172456.9 & 10.304 & 9.777 & 6.744 & 3.229 & Classe II \\
\hline 95 & 13.2126 & 0.0974 & J181357.00-172543.1 & 9.804 & 9.485 & 7.280 & 3.962 & Classe II \\
\hline 96 & 13.2128 & 0.1152 & J181353.08-172511.7 & 10.206 & 9.920 & 7.349 & 4.207 & Classe II \\
\hline 97 & 13.2164 & 0.1159 & J181353.36-172459.1 & 9.300 & 10.050 & 8.821 & 4.499 & Classe II \\
\hline 98 & 13.2433 & 0.0710 & J181406.52-172451.6 & 9.465 & 9.015 & 6.765 & 3.092 & Classe II \\
\hline 99 & 13.2482 & 0.0971 & J181401.34-172351.1 & 9.776 & 9.168 & 8.050 & 4.627 & Classe II \\
\hline 100 & 13.2679 & 0.0722 & $\mathrm{~J} 181409.21-172331.5$ & 11.125 & 10.261 & 7.874 & 4.907 & Classe II \\
\hline 101 & 13.2659 & 0.0771 & J181407.89-172329.7 & 9.496 & 9.243 & 7.583 & 4.824 & Classe II \\
\hline 102 & 13.2420 & 0.1051 & J181358.82-172356.7 & 10.622 & 10.187 & 7.297 & 4.686 & Classe II \\
\hline 103 & 13.2390 & 0.1185 & J181355.51-172343.3 & 10.482 & 9.921 & 7.358 & 4.132 & Classe II \\
\hline 104 & 13.1380 & -0.0254 & J181415.16-173310.3 & 11.555 & 11.301 & 8.303 & 6.554 & Disco de Transição \\
\hline 105 & 13.1131 & -0.0255 & $\mathrm{~J} 181412.18-173429.2$ & 9.828 & 9.621 & 8.645 & 7.095 & Disco de Transição \\
\hline 106 & 13.1302 & -0.0333 & J181415.95-173348.5 & 9.932 & 9.692 & 8.278 & 6.252 & Disco de Transição \\
\hline 107 & 13.1319 & -0.0260 & J181414.56-173330.7 & 9.684 & 9.453 & 8.130 & 5.958 & Disco de Transição \\
\hline 108 & 13.1630 & -0.0390 & $\mathrm{~J} 181421.17-173214.8$ & 8.383 & 7.905 & 7.722 & 5.794 & Disco de Transição \\
\hline 109 & 13.1434 & -0.0357 & J181418.08-173311.0 & 10.852 & 10.078 & 9.131 & 7.067 & Disco de Transição \\
\hline 110 & 13.1559 & -0.0304 & J181418.41-173222.5 & 8.913 & 8.355 & 7.577 & 5.329 & Disco de Transição \\
\hline 111 & 13.1591 & -0.0291 & $\mathrm{~J} 181418.52-173210.1$ & 8.583 & 8.237 & 8.206 & 6.513 & Disco de Transição \\
\hline 112 & 13.1685 & -0.0357 & J181421.10-173151.7 & 9.228 & 8.592 & 8.107 & 6.014 & Disco de Transição \\
\hline 113 & 13.1178 & -0.0041 & J181408.01-173337.4 & 10.642 & 9.930 & 9.228 & 6.738 & Disco de Transição \\
\hline 114 & 13.1414 & -0.0040 & J181410.84-173222.7 & 11.260 & 10.857 & 7.784 & 5.008 & Disco de Transição \\
\hline 115 & 13.1429 & -0.0156 & J181413.57-173237.9 & 8.265 & 8.045 & 8.394 & 6.546 & Disco de Transição \\
\hline 116 & 13.1471 & 0.0039 & J181409.76-173151.2 & 10.965 & 10.559 & 7.662 & 5.014 & Disco de Transição \\
\hline 117 & 13.1338 & 0.0047 & J181408.00-173231.7 & 11.836 & 11.295 & 8.002 & 5.328 & Disco de Transição \\
\hline
\end{tabular}


Tabela A.1 - continua

\begin{tabular}{|c|c|c|c|c|c|c|c|c|}
\hline \multirow{2}{*}{$\begin{array}{c}\text { Candidata } \\
\#\end{array}$} & \multirow{2}{*}{$\begin{array}{c}1 \\
{\left[{ }^{\circ}\right]}\end{array}$} & \multirow{2}{*}{$\begin{array}{c}\mathrm{b} \\
{\left[{ }^{\circ}\right]}\end{array}$} & \multirow{2}{*}{$\begin{array}{l}\text { Identificação } \\
\text { WISE }\end{array}$} & \multicolumn{4}{|c|}{ Fluxos (mag) } & \multirow[t]{2}{*}{ Classificação } \\
\hline & & & & {$[3.4 \mu \mathrm{m}]$} & {$[4.6 \mu \mathrm{m}]$} & {$[12 \mu \mathrm{m}]$} & {$[22 \mu \mathrm{m}]$} & \\
\hline 118 & 13.1148 & 0.0518 & J181355.31-173210.8 & 11.336 & 11.114 & 7.206 & 5.035 & Disco de Transição \\
\hline 119 & 13.1511 & 0.0367 & J181403.02-173042.0 & 10.622 & 10.453 & 9.851 & 5.419 & Disco de Transição \\
\hline 120 & 13.1361 & 0.0203 & J181404.83-173157.6 & 7.942 & 7.328 & 6.682 & 4.696 & Disco de Transição \\
\hline 121 & 13.1737 & -0.0160 & J181417.38-173101.2 & 11.805 & 11.355 & 8.017 & 4.768 & Disco de Transição \\
\hline 122 & 13.1491 & -0.0062 & J181412.24-173202.1 & 9.232 & 9.026 & 7.356 & 4.807 & Disco de Transição \\
\hline 123 & 13.1801 & -0.0195 & J181418.90-173047.1 & 9.321 & 8.778 & 7.815 & 5.611 & Disco de Transição \\
\hline 124 & 13.1889 & -0.0263 & J181421.47-173031.1 & 11.226 & 10.936 & 7.854 & 6.182 & Disco de Transição \\
\hline 125 & 13.1775 & -0.0162 & J181417.88-173049.7 & 10.607 & 10.353 & 7.602 & 4.404 & Disco de Transição \\
\hline 126 & 13.1810 & -0.0299 & J181421.31-173102.2 & 12.121 & 11.943 & 8.638 & 5.979 & Disco de Transição \\
\hline 127 & 13.1864 & -0.0094 & J181417.43-173009.7 & 10.567 & 10.264 & 9.263 & 6.273 & Disco de Transição \\
\hline 128 & 13.1724 & 0.0203 & J181409.19-173002.7 & 11.192 & 10.765 & 5.473 & 1.320 & Disco de Transição \\
\hline 129 & 13.1668 & 0.0212 & J181408.31-173019.1 & 10.302 & 9.588 & 5.375 & 0.938 & Disco de Transição \\
\hline 130 & 13.1777 & 0.0131 & J181411.42-172958.4 & 10.984 & 10.516 & 9.688 & 3.001 & Disco de Transição \\
\hline 131 & 13.1698 & 0.0259 & J181407.64-173001.4 & 9.631 & 9.229 & 5.086 & 0.549 & Disco de Transição \\
\hline 132 & 13.1665 & 0.0306 & J181406.20-173003.7 & 10.611 & 9.836 & 4.188 & -0.772 & Disco de Transição \\
\hline 133 & 13.1861 & 0.0172 & J181411.53-172925.0 & 7.226 & 6.464 & 5.898 & 1.992 & Disco de Transição \\
\hline 134 & 13.2099 & 0.0177 & J181414.29-172808.9 & 11.603 & 10.824 & 5.747 & 1.936 & Disco de Transição \\
\hline 135 & 13.1981 & 0.0108 & J181414.38-172858.1 & 11.703 & 11.179 & 6.253 & 1.919 & Disco de Transição \\
\hline 136 & 13.2055 & 0.0025 & $\mathrm{~J} 181417.10-172849.0$ & 11.824 & 11.332 & 7.058 & 3.638 & Disco de Transição \\
\hline 137 & 13.2126 & 0.0194 & J181414.22-172757.5 & 12.147 & 11.623 & 6.538 & 1.997 & Disco de Transição \\
\hline 138 & 13.1858 & 0.0049 & J181414.20-172947.0 & 10.778 & 10.507 & 7.445 & 4.071 & Disco de Transição \\
\hline 139 & 13.1303 & 0.0691 & J181353.35-173052.1 & 9.467 & 9.299 & 7.496 & 4.097 & Disco de Transição \\
\hline 140 & 13.1286 & 0.0610 & J181354.93-173111.4 & 10.033 & 9.571 & 4.676 & 2.042 & Disco de Transição \\
\hline 141 & 13.1316 & 0.0521 & J181357.26-173117.1 & 10.701 & 10.087 & 6.685 & 3.236 & Disco de Transição \\
\hline 142 & 13.1122 & 0.0773 & J181349.34-173135.1 & 10.111 & 9.883 & 10.262 & 7.892 & Disco de Transição \\
\hline 143 & 13.1187 & 0.0564 & J181354.75-173150.5 & 11.499 & 11.298 & 6.807 & 4.052 & Disco de Transição \\
\hline 144 & 13.1589 & 0.0387 & $\mathrm{~J} 181403.50-173014.0$ & 9.631 & 8.931 & 5.122 & 0.934 & Disco de Transição \\
\hline 145 & 13.1421 & 0.0526 & $\mathrm{~J} 181358.42-173043.0$ & 11.134 & 10.699 & 6.780 & 3.402 & Disco de Transição \\
\hline 146 & 13.1280 & 0.0967 & J181346.96-173011.5 & 9.944 & 9.647 & 8.528 & 6.409 & Disco de Transição \\
\hline 147 & 13.1250 & 0.0795 & J181350.40-173050.9 & 8.393 & 8.130 & 7.594 & 5.493 & Disco de Transição \\
\hline 148 & 13.1524 & 0.0761 & J181354.44-172930.1 & 8.655 & 8.281 & 7.398 & 5.147 & Disco de Transição \\
\hline 149 & 13.1753 & 0.0488 & J181403.24-172904.5 & 9.535 & 8.937 & 2.135 & -2.611 & Disco de Transição \\
\hline 150 & 13.1832 & 0.0570 & J181402.39-172825.6 & 10.112 & 9.869 & 3.703 & -0.608 & Disco de Transição \\
\hline 151 & 13.1712 & 0.0418 & J181404.30-172929.5 & 10.602 & 10.088 & 3.017 & -1.599 & Disco de Transição \\
\hline 152 & 13.1826 & 0.0528 & J181403.24-172834.6 & 9.907 & 9.260 & 1.655 & -2.432 & Disco de Transição \\
\hline 153 & 13.1639 & 0.0376 & $\mathrm{~J} 181404.35-173000.0$ & 10.127 & 9.568 & 3.913 & -0.276 & Disco de Transição \\
\hline 154 & 13.1713 & 0.0395 & J181404.81-172933.3 & 11.091 & 10.374 & 3.963 & -0.653 & Disco de Transição \\
\hline 155 & 13.2105 & 0.0229 & $\mathrm{~J} 181413.21-172758.0$ & 11.888 & 11.416 & 5.427 & 1.524 & Disco de Transição \\
\hline 156 & 13.2038 & 0.0461 & J181407.28-172739.2 & 10.341 & 9.920 & 4.262 & -0.182 & Disco de Transição \\
\hline 157 & 13.2089 & 0.0266 & J181412.20-172756.8 & 11.358 & 10.652 & 5.704 & 0.822 & Disco de Transição \\
\hline 158 & 13.2130 & 0.0355 & J181410.72-172728.4 & 10.631 & 10.347 & 4.588 & 1.039 & Disco de Transição \\
\hline 159 & 13.2103 & 0.0242 & J181412.89-172756.1 & 11.682 & 10.981 & 5.123 & 1.075 & Disco de Transição \\
\hline 160 & 13.1939 & 0.0384 & J181407.78-172823.8 & 10.681 & 9.957 & 2.256 & -2.989 & Disco de Transição \\
\hline 161 & 13.1896 & 0.0791 & J181358.26-172727.3 & 10.666 & 10.425 & 6.035 & 3.807 & Disco de Transição \\
\hline
\end{tabular}


Tabela A.1 - continua

\begin{tabular}{|c|c|c|c|c|c|c|c|c|}
\hline \multirow{2}{*}{$\begin{array}{c}\text { Candidata } \\
\#\end{array}$} & \multirow{2}{*}{$\begin{array}{c}1 \\
{\left[{ }^{\mathrm{o}}\right]}\end{array}$} & \multirow{2}{*}{$\begin{array}{c}\mathrm{b} \\
{\left[{ }^{\circ}\right]}\end{array}$} & \multirow{2}{*}{$\begin{array}{c}\text { Identificação } \\
\text { WISE }\end{array}$} & \multicolumn{4}{|c|}{ Fluxos (mag) } & \multirow[t]{2}{*}{ Classificação } \\
\hline & & & & {$[3.4 \mu \mathrm{m}]$} & {$[4.6 \mu \mathrm{m}]$} & {$[12 \mu \mathrm{m}]$} & {$[22 \mu \mathrm{m}]$} & \\
\hline 162 & 13.1801 & 0.0761 & J181357.79-172802.5 & 9.975 & 9.662 & 6.309 & 3.485 & Disco de Transição \\
\hline 163 & 13.1976 & 0.0803 & J181358.98-172659.8 & 11.393 & 10.948 & 7.773 & 3.752 & Disco de Transição \\
\hline 164 & 13.1915 & 0.0868 & J181356.80-172708.0 & 11.358 & 10.833 & 6.953 & 4.164 & Disco de Transição \\
\hline 165 & 13.1984 & 0.0722 & $\mathrm{~J} 181400.85-172711.2$ & 11.740 & 11.317 & 6.627 & 3.467 & Disco de Transição \\
\hline 166 & 13.1974 & 0.0662 & J181402.06-172724.8 & 10.170 & 9.973 & 5.734 & 1.855 & Disco de Transição \\
\hline 167 & 13.2222 & 0.0750 & J181403.09-172551.2 & 9.327 & 8.771 & 8.291 & 2.102 & Disco de Transição \\
\hline 168 & 13.2081 & 0.0561 & J181405.57-172708.5 & 10.739 & 10.058 & 4.434 & 0.552 & Disco de Transição \\
\hline 169 & 13.2017 & -0.0300 & J181423.83-172956.8 & 11.641 & 11.337 & 7.013 & 4.566 & Disco de Transição \\
\hline 170 & 13.2052 & -0.0235 & J181422.82-172934.6 & 11.378 & 11.212 & 7.666 & 5.331 & Disco de Transição \\
\hline 171 & 13.2266 & -0.0156 & J181423.66-172813.2 & 11.528 & 11.375 & 8.565 & 5.659 & Disco de Transição \\
\hline 172 & 13.2147 & -0.0087 & J181420.69-172839.2 & 11.950 & 11.775 & 8.072 & 5.042 & Disco de Transição \\
\hline 173 & 13.2250 & 0.0111 & J181417.54-172732.5 & 10.599 & 10.406 & 8.002 & 5.212 & Disco de Transição \\
\hline 174 & 13.2556 & -0.0021 & J181424.15-172618.2 & 10.899 & 10.461 & 9.358 & 5.608 & Disco de Transição \\
\hline 175 & 13.2439 & 0.0016 & J181421.94-172648.9 & 10.058 & 9.892 & 6.862 & 4.494 & Disco de Transição \\
\hline 176 & 13.2406 & 0.0040 & J181421.00-172655.2 & 11.241 & 11.043 & 6.649 & 4.894 & Disco de Transição \\
\hline 177 & 13.2484 & 0.0094 & J181420.74-172621.2 & 11.502 & 11.349 & 7.695 & 5.089 & Disco de Transição \\
\hline 178 & 13.2676 & 0.0133 & $\mathrm{~J} 181422.20-172514.1$ & 9.564 & 9.109 & 8.739 & 6.273 & Disco de Transição \\
\hline 179 & 13.2318 & 0.0604 & J181407.47-172546.1 & 9.299 & 9.099 & 7.004 & 4.299 & Disco de Transição \\
\hline 180 & 13.2297 & 0.0737 & J181404.30-172529.9 & 9.594 & 9.317 & 4.814 & 1.510 & Disco de Transição \\
\hline 181 & 13.2416 & 0.0659 & J181407.44-172505.7 & 10.003 & 9.658 & 6.606 & 3.980 & Disco de Transição \\
\hline 182 & 13.2338 & 0.0719 & J181405.18-172519.8 & 12.536 & 11.969 & 5.976 & 1.548 & Disco de Transição \\
\hline 183 & 13.2315 & 0.0551 & J181408.63-172556.0 & 10.239 & 10.048 & 7.724 & 5.911 & Disco de Transição \\
\hline 184 & 13.2344 & 0.0724 & J181405.15-172517.3 & 12.369 & 11.834 & 5.563 & 1.525 & Disco de Transição \\
\hline 185 & 13.2363 & 0.0654 & J181406.92-172523.1 & 10.862 & 10.665 & 6.555 & 3.011 & Disco de Transição \\
\hline 186 & 13.1462 & 0.1193 & J181344.17-172835.2 & 9.092 & 8.479 & 7.845 & 5.891 & Disco de Transição \\
\hline 187 & 13.1188 & 0.1113 & J181342.64-173015.7 & 10.204 & 9.787 & 8.924 & 6.601 & Disco de Transição \\
\hline 188 & 13.1502 & 0.1042 & J181347.97-172848.4 & 9.896 & 9.186 & 8.720 & 6.425 & Disco de Transição \\
\hline 189 & 13.1501 & 0.1144 & J181345.71-172831.4 & 9.305 & 9.117 & 8.257 & 6.510 & Disco de Transição \\
\hline 190 & 13.1924 & 0.0975 & J181354.55-172646.7 & 9.473 & 9.250 & 8.793 & 4.548 & Disco de Transição \\
\hline 191 & 13.1979 & 0.1120 & J181352.00-172604.4 & 8.698 & 8.412 & 8.768 & 6.937 & Disco de Transição \\
\hline 192 & 13.1957 & 0.0886 & J181356.90-172651.6 & 11.635 & 11.142 & 6.151 & 3.423 & Disco de Transição \\
\hline 193 & 13.2292 & 0.0910 & J181400.42-172501.5 & 11.600 & 11.348 & 6.060 & 1.161 & Disco de Transição \\
\hline 194 & 13.2239 & 0.0903 & J181359.92-172519.4 & 12.463 & 11.933 & 7.178 & 1.502 & Disco de Transição \\
\hline 195 & 13.2226 & 0.1037 & J181356.81-172500.8 & 11.410 & 10.976 & 6.355 & 3.515 & Disco de Transição \\
\hline 196 & 13.2203 & 0.1024 & J181356.83-172510.0 & 10.970 & 10.501 & 5.847 & 3.271 & Disco de Transição \\
\hline 197 & 13.2295 & 0.0835 & J181402.10-172513.4 & 11.661 & 11.143 & 5.267 & 0.385 & Disco de Transição \\
\hline 198 & 13.2246 & 0.1026 & J181357.29-172456.0 & 10.644 & 10.153 & 5.588 & 3.409 & Disco de Transição \\
\hline 199 & 13.2298 & 0.0885 & J181401.03-172504.1 & 11.742 & 11.114 & 5.584 & 1.075 & Disco de Transição \\
\hline 200 & 13.2252 & 0.0813 & J181402.07-172530.9 & 10.578 & 10.153 & 6.911 & 0.759 & Disco de Transição \\
\hline 201 & 13.2284 & 0.1016 & J181357.97-172445.8 & 12.267 & 11.619 & 5.539 & 3.212 & Disco de Transição \\
\hline 202 & 13.2280 & 0.0838 & J181401.85-172517.7 & 11.410 & 11.135 & 5.899 & 0.356 & Disco de Transição \\
\hline 203 & 13.2127 & 0.0831 & J181400.16-172607.3 & 8.735 & 8.405 & 7.955 & 4.510 & Disco de Transição \\
\hline 204 & 13.2229 & 0.0911 & J181359.62-172521.4 & 11.294 & 10.889 & 7.497 & 2.031 & Disco de Transição \\
\hline 205 & 13.2372 & 0.1141 & J181356.27-172356.5 & 8.749 & 8.517 & 7.132 & 4.033 & Disco de Transição \\
\hline
\end{tabular}


Tabela A.1 - continua

\begin{tabular}{|c|c|c|c|c|c|c|c|c|}
\hline \multirow{2}{*}{$\begin{array}{c}\text { Candidata } \\
\#\end{array}$} & \multirow{2}{*}{$\begin{array}{c}1 \\
{\left[{ }^{\circ}\right]}\end{array}$} & \multirow{2}{*}{$\begin{array}{c}\mathrm{b} \\
{\left[{ }^{\circ}\right]}\end{array}$} & \multirow{2}{*}{$\begin{array}{l}\text { Identificação } \\
\text { WISE }\end{array}$} & \multicolumn{4}{|c|}{ Fluxos (mag) } & \multirow[t]{2}{*}{ Classificação } \\
\hline & & & & {$[3.4 \mu \mathrm{m}]$} & {$[4.6 \mu \mathrm{m}]$} & {$[12 \mu \mathrm{m}]$} & {$[22 \mu \mathrm{m}]$} & \\
\hline 206 & 13.2136 & 0.1166 & J181352.87-172507.0 & 11.056 & 10.724 & 7.227 & 4.170 & Disco de Transição \\
\hline 207 & 13.2205 & 0.1102 & J181355.12-172455.8 & 10.310 & 10.150 & 7.548 & 4.420 & Disco de Transição \\
\hline 208 & 13.2377 & 0.0983 & J181359.81-172422.0 & 12.059 & 11.550 & 6.093 & 3.520 & Disco de Transição \\
\hline 209 & 13.2404 & 0.0764 & J181404.99-172451.4 & 9.730 & 9.387 & 4.296 & 1.688 & Disco de Transição \\
\hline 210 & 13.2385 & 0.0818 & J181403.55-172448.1 & 10.547 & 9.932 & 4.625 & 1.282 & Disco de Transição \\
\hline 211 & 13.2315 & 0.0864 & $\mathrm{~J} 181401.70-172502.2$ & 12.504 & 12.291 & 5.677 & 0.618 & Disco de Transição \\
\hline 212 & 13.2323 & 0.0929 & J181400.37-172448.5 & 11.057 & 10.655 & 6.449 & 2.195 & Disco de Transição \\
\hline 213 & 13.2312 & 0.0824 & $\mathrm{~J} 181402.55-172510.2$ & 10.613 & 9.946 & 4.475 & -0.268 & Disco de Transição \\
\hline 214 & 13.2292 & 0.0787 & J181403.12-172522.9 & 9.237 & 9.014 & 3.736 & 0.416 & Disco de Transição \\
\hline 215 & 13.2371 & 0.0936 & J181400.78-172432.1 & 10.320 & 9.876 & 5.402 & 2.454 & Disco de Transição \\
\hline 216 & 13.2325 & 0.0770 & J181403.89-172515.4 & 10.064 & 9.692 & 5.063 & -0.432 & Disco de Transição \\
\hline 217 & 13.2404 & 0.0940 & J181401.08-172421.0 & 9.943 & 9.723 & 4.805 & 3.131 & Disco de Transição \\
\hline 218 & 13.2435 & 0.0771 & J181405.21-172440.2 & 11.152 & 10.697 & 6.127 & 2.319 & Disco de Transição \\
\hline 219 & 13.2468 & 0.0740 & J181406.27-172435.2 & 11.786 & 11.424 & 7.352 & 4.218 & Disco de Transição \\
\hline 220 & 13.2352 & 0.0992 & J181359.33-172428.5 & 9.378 & 9.056 & 5.712 & 2.634 & Disco de Transição \\
\hline 221 & 13.2516 & 0.0858 & J181404.24-172359.7 & 8.614 & 8.314 & 7.176 & 4.687 & Disco de Transição \\
\hline 222 & 13.2427 & 0.0814 & J181404.14-172435.4 & 10.319 & 10.027 & 4.899 & 2.521 & Disco de Transição \\
\hline 223 & 13.2390 & 0.0928 & J181401.19-172427.4 & 9.106 & 8.818 & 5.859 & 2.362 & Disco de Transição \\
\hline 224 & 13.2471 & 0.0781 & $\mathrm{~J} 181405.40-172427.1$ & 10.256 & 10.098 & 6.963 & 3.952 & Disco de Transição \\
\hline 225 & 13.2354 & 0.0725 & J181405.22-172513.9 & 10.123 & 9.731 & 4.448 & 1.682 & Disco de Transição \\
\hline 226 & 13.2422 & 0.0887 & J181402.49-172424.4 & 10.677 & 10.362 & 5.643 & 2.381 & Disco de Transição \\
\hline 227 & 13.2676 & 0.0821 & J181407.00-172315.7 & 10.238 & 10.018 & 7.799 & 5.071 & Disco de Transição \\
\hline 228 & 13.2696 & 0.0742 & J181408.98-172322.8 & 10.599 & 10.386 & 7.460 & 4.081 & Disco de Transição \\
\hline 229 & 13.2668 & 0.0850 & $\mathrm{~J} 181406.25-172313.1$ & 10.240 & 9.574 & 8.588 & 5.451 & Disco de Transição \\
\hline 230 & 13.2589 & 0.0796 & J181406.49-172347.4 & 7.302 & 6.825 & 6.675 & 4.609 & Disco de Transição \\
\hline 231 & 13.2457 & 0.1019 & J181400.00-172350.7 & 9.816 & 9.327 & 10.194 & 5.108 & Disco de Transição \\
\hline 232 & 13.2399 & 0.1093 & J181357.66-172356.3 & 9.114 & 8.881 & 7.718 & 4.412 & Disco de Transição \\
\hline 233 & 13.2487 & 0.1005 & J181400.65-172343.4 & 10.996 & 10.360 & 11.104 & 6.363 & Disco de Transição \\
\hline 234 & 13.2372 & 0.1034 & J181358.63-172415.1 & 9.272 & 8.958 & 6.258 & 3.162 & Disco de Transição \\
\hline
\end{tabular}

